\title{
LIGHT AND SHADOW EXPRESSION:
}

Contrast, Luminosity, Porosity

Victor Hieu Huynh

Bachelor of Architectural Science, Ryerson University, 2014.

\author{
A Thesis \\ presented to Ryerson University \\ in partial fulfillment of the \\ requirements for the degree of \\ Master of Architecture \\ in the program of \\ Architecture \\ Toronto, Ontario, Canada, 2018 \\ (C) Victor Huynh 2018
}





\section{Author's Declaration}

I hereby declare that I am the sole author of this thesis. This is a true copy of the thesis, including any required final revisions, as accepted by my examiners.

I authorize Ryerson University to lend this thesis project to other institutions or individuals for the purpose of scholarly research.

I further authorize Ryerson University to reproduce this thesis project by photocopying or by other means, in total or in part, at the request of other institutions or individuals for the purpose of scholarly research.

I understand that my thesis may be electronically available to the public.

Victor Huynh 



\section{Abstract}

The pervasive use of glass curtain wall and artificial illumination has saturated contemporary architecture with light, depriving users of the potential experiences achieved through the articulation and interplay of light and shadow. The evaluation of light through quantifiable metric design evaluation denies users of qualitative lighting design expression that influences spatial perception and user experience, engaging the eye, mind and body over the course of the day. Homogeneous and standard lighting solutions deny users sensorial and perceptual stimulation developed through lighting variability and contrast.

This thesis reconciles the use of light and shadow as an architectural design medium to support and enrich the mundane office working environment, establishing a relationship between light, the built environment, and its users. The expression of sunlight, artificial light, architectural form, the physical properties of material, and spatial ambiance improves the architectural experience and spatial perception throughout the day. 



\section{Acknowledgment}

I would like to thank and express my sincere gratitude to the development of my thesis project towards the following:

Vincent Hui,

Tom Bessai,

Miljana Horvat,

Friends and Family 

To my family and friends 
x | Light and Shadow Expression 


\title{
Table of Contents
}

\author{
Authors Declaration \\ Abstract \\ Acknowledgments \\ Dedication \\ List of Figures \\ Introduction
}

Chapter $1 \quad$ Discussing Light

Understanding Light 05

The Human Body, Perception and Experience 11

Chapter 2 Contrast

Historical Application $\quad 21$

In Praise of the Shadow 25

Chapter 3 Luminosity

Modern Transformation 33

Luminous Environment $\quad 37$

Chapter 4 Porosity

Contemporary Paradigm 43

Experiencing the Ephemeral 51

Chapter $5 \quad$ Light and Architecture

Architecture as a Vehicle to express Light 59

Lighting Design Strategies 60

Site Description 65

Programmatic Description $\quad 68$

Design Project $\quad 73$

$\begin{array}{ll}\text { Conclusion } & 98\end{array}$

Appendix A

Light and Shadow Experiments 103

Appendix B

Additional Project Drawings 113

Appendix C

Day/Night Exterior Lighting Condition 119

$\begin{array}{ll}\text { Bibliography } & 123\end{array}$ 
xii | Light and Shadow Expression 


\section{List of Figures}

Figure 00

Paper Works

Source https://www.dezeen.com/2012/05/28/paper-works-bysimon-schubert/

Figure $01 \quad$ Electromagnetic Radiation

Source https://imagine.gsfc.nasa.gov/science/toolbox/

emspectrum 1.html

Figure 02 Light Prism Experiment Newton

Source https://www.artofclaytaylor.com/single-

post/2016/08/22/The-Magnetic-Spectrum-of-Inertial-

Polarization

Figure 03 Light Prism Experiment Goethe

Source https://www.artofclaytaylor.com/single-

post/2016/08/22/The-Magnetic-Spectrum-of-Inertial-

Polarization

Figure 04 Light's Behaviour

Source http://weeklysciencequiz.blogspot.ca/2011/09/whenlight-meets-matter.html

Figure 05 Material Characteristics

Source: A http://ynedpc.com/projects/lvmh/

B https://www.archdaily.com/54199/glass-pavilion-at-the-

toledo-museum-of-art-sanaa-pritzker-prize-2010

C https://smarthistory.org/frank-gehry-guggenheim-

bilbao/

D http://ming3d.com/DAAP/ARCH794su2011/?author=10 
Figure $06 \quad$ Material and Light Experiment

Source Victor H. Huynh

Figure 07 Stone Wall Construction

Source: A https://www.livingstonemasons.com/instructional/howto-build-a-drystone-retaining-wall

B https://anjalimangalgiri.wordpress.com/2014/02/20/thedisappearing-winery/

Figure 08 Daylight Transformation

Source http://www.t5fixtures.com/daylight-full-spectrum-t5-

lights/\#prettyPhoto

Figure 09 Circadian Rhythm

Source https://en.wikipedia.org/wiki/Circadian_rhythm\#/media/

File:Biological_clock_human.svg

Figure 10 Melatonin Levels

Source https://www.howsleepworks.com/how_circadian.html

Figure 11 Tindaya - Eduardo Chillida

Source http://www.floornature.com/montana-tindaya-eduardochillida-fuerteventura-spain-4102/

Figure 12 Understanding Perception

Source Victor Huynh

Figure 13 Light \& Shadow Revealing Form

Source http://architecturedesignentrance.blogspot.ca/2012/04/

light-and-shadows.html

Figure 14 Ganzfeld - James Turrel

Source http://jamesturrell.com/work/type/ganzfeld/

Figure 15 Pyramid of Giza

Source http://www.starseeds.net/group/ alternativearchaeologyforbiddenhistory/forum/topics/

the-great-pyramid-at-giza

Figure 16 Chiaroscuro

Source https://www.widewalls.ch/chiaroscuro/

Figure 17 Pantheon

Source http://www.architecturerevived.com/the-pantheonromes-architecture-of-the-cosmos/ 
Figure 18

Source: A

\section{B}

Figure 19

Source

Figure 20

Source

Figure 21

Source

Figure 22

Source: A

B

Figure 23

Source

Figure 24

Source

Figure 25

Source

Figure 26

Source

Figure 27

Source

Figure 28

Source

Figure 29

Source
Stained Glass Window / Chartres Cathedral https://en.wikipedia.org/wiki/Chartres_Cathedral https://en.wikipedia.org/wiki/Stained_glass

Ecstasy of Saint Teresa

http://www.learner.org/courses/globalart/work/98/

Japanese House Lighting Design

http://iva.velux.com/competitions/international-veluxaward-2018/posts/light-and-materials

Light and Shadow Patterns

https://www.pinterest.ca/pin/36169603229489753/

Church of the Light - Tado Ando

http://www.izinsizgosteri.net/

new $/$ ?page $=1 \&$ content $=585$

https://www.pinterest.ca/pin/812829432716926330/

Ronchomp, Le Corbusier

https://www.archdaily.com/84988/ad-classics-ronchample-corbusier

Villa Savoye - Le Corbusier https://www.archdaily.com/84524/ad-classics-villasavoye-le-corbusier

Textured vs Flat Surface http://interiorzine.com/2016/01/25/cesello-collectionby-raffaello-galiotto-for-lithos-design-domino/

Crystal Palace https://blackcablondon.net/2013/04/07/crystal-palacepark-part-one/

Glass House - Philip Johnson

https://en.wikipedia.org/wiki/Glass_House

Glass Museum - Sanaa

https://www.archdaily.com/54199/glass-pavilion-at-thetoledo-museum-of-art-sanaa-pritzker-prize-2010

The Seagram Building - Mies Van de Rohe https://www.archdaily.com/501008/light-matters-richardkelly-the-unsung-master-behind-modern-architecture-s- 
greatest-buildings

Figure 30 Lighting Effects seen in the Natural Environment

Source: A https://www.sciencenewsforstudents.org/article/cooljobs-bringing-caves-dark-secrets-light

B https://www.quora.com/What-is-the-differencebetween-foggy-and-misty

C https://www.tes.com/lessons/vEE9_LnB8KyiqA/copy-ofaurora-borealis

Figure 31 Architectural Lighting Design - Marilyne Andersen

Source http://infoscience.epfl.ch/record/186396/files/LUX\%20

EUROPA\%202013_Rockcastle\%20\%20Andersen_FINAL. pdf?version $=137$

Figure 32 Lighting Typology - Marilyne Andersen

Source http://infoscience.epfl.ch/record/186396/files/LUX\%20

EUROPA\%202013_Rockcastle\%20\%20Andersen_FINAL. pdf?version $=140$

Figure 33 Tate Modern Museum - Herzog de Meuron

Source: A https://traveldigg.com/tate-modern-the-modern-artgallery-located-in-london/

B The Weather Project - Olsfar Eliasson http://olafureliasson.net/archive/exhibition/EXH101069/ the-weather-project

Figure 34 Day to Night Transformation in a City

Source https://www.whudat.de/amazing-time-slicephotography-by-michael-marker-moore-6-pictures/

Figure 35 Chapel of St. Ignatius - Steven Holl

Source http://www.stevenholl.com/projects/st-ignatius-chapel

Figure 36 Periscope Window - James Carpenter

Source http://www.jcdainc.com/projects/periscope-window

Figure 37 Sarphatistraat Office - Steven Holl

Source http://www.stevenholl.com/projects/sarphatistraatoffices

Figure 38 Atkins Museum - Steven Holl

Source Photos by Andy Ryan, https://www.archdaily.com/4369/ the-nelson-atkins-museum-of-art-steven-holl-architects 
Figure 39

Sunlight Integration

Source: A https://www.wired.com/2009/10/1006lecorbusier-born/

B http://www.stevenholl.com/projects/st-ignatius-chapel

Figure 40

Artificial Light Expression

Source: A https://www.dezeen.com/2013/01/19/light-pavilion-bylebbeus-woods-at-sliced-porosity-block/

B https://www.archdaily.com/118627/ad-classics-sendaimediatheque-toyo-ito

Figure $41 \quad$ Architectural Form and Light

Source: A https://www.archdaily.com/597598/light-matters-lecorbusier-and-the-trinity-of-light

B https://www.inexhibit.com/mymuseum/menil-collectionhouston-texas/

Figure 42 Material Properties and Light

Source: A http://www.amusingplanet.com/2014/09/beinecke-rarebook-and-manuscript.html

B https://www.dezeen.com/2007/09/09/dominus-wineryby-herzog-de-meuron/

Figure 43

Source: A

Spatial Ambiance http://oma.eu/projects/seattle-central-library

B https://www.pinterest.ca/pin/513199320019198144/

Figure 44 Site Location

Source Victor H. Huynh

Figure 45 Site Photos

Source Victor H. Huynh

Figure $46 \quad$ Neighbourhood Photos

Source Victor H. Huynh

Figure 47 Centre for Social Innovation

Source https://socialinnovation.org/location/csispadina/

Figure 48 The Office Environment

Source http://www.movehut.co.uk/news/20th-vs-21st-centuryoffices-how-far-have-we-come-34104/ 
Figure 49 Natural Environment Lighting Effects

Source: A https://fineartamerica.com/featured/forest-light-rays-bonielsen.html

B https://blog.photoshopcreative.co.uk/blog/tutorials/ how-to-make-rays-of-light-in-photoshop/

C cave light

https://top10vietnam.wordpress.com/02-light/

Figure 50 Orthographic Drawing

Source: a Programmatic Organization

Victor H. Huynh

b Ground Floor Plan

Source Victor H. Huynh

c Second Floor Plan

Source Victor H. Huynh

d Third Floor Plan

Source Victor H. Huynh

Figure 51 Exterior Perspective

a Exterior View

Source Victor H. Huynh

b Hallway

Source Victor H. Huynh

c Kitchen

Source Victor H. Huynh

d Office Work Station 1

Source Victor H. Huynh

e Collaborative Space 1

Source Victor H. Huynh

f Collaborative Space 2

Source Victor H. Huynh

$9 \quad$ Office Work Station 2

Source Victor H. Huynh

h Private Meeting Room

Source Victor H. Huynh 


\begin{tabular}{|c|c|}
\hline i & Office Work Station 3 \\
\hline Source & Victor H. Huynh \\
\hline j & Street Perspective \\
\hline Source & Victor H. Huynh \\
\hline k & Street Perspective \\
\hline Source & Victor H. Huynh \\
\hline Figure 52 & Orthographic Drawing \\
\hline$a$ & Section A-A \\
\hline Source & Victor H. Huynh \\
\hline$b$ & Section B-B \\
\hline Source & Victor H. Huynh \\
\hline $\mathrm{C}$ & Section C-C \\
\hline Source & Victor H. Huynh \\
\hline$d$ & Section D-D \\
\hline Source & Victor H. Huynh \\
\hline $\begin{array}{r}\text { Figure } 53 \\
\text { a }\end{array}$ & Perspective Rendering Sequence \\
\hline Source & Victor H. Huynh \\
\hline$b$ & Kitchen \\
\hline Source & Victor H. Huynh \\
\hline C & Office Work Station 1 \\
\hline Source & Victor H. Huynh \\
\hline$d$ & Collaborative Space 1 \\
\hline Source & Victor H. Huynh \\
\hline e & Collaborative Space 2 \\
\hline Source & Victor H. Huynh \\
\hline$f$ & Office Work Station 2 \\
\hline Source & Victor H. Huynh \\
\hline $\begin{array}{r}9 \\
\text { Source }\end{array}$ & $\begin{array}{l}\text { Private Meeting Room } \\
\text { Victor H. Huynh }\end{array}$ \\
\hline
\end{tabular}




\begin{tabular}{|c|c|}
\hline $\mathrm{h}$ & Office Work Station 3 \\
\hline Source & Victor H. Huynh \\
\hline Figure 54 & Rendering Compilation \\
\hline Source & Victor H. Huynh \\
\hline Figure 55 & CNC Aperture Experiment \\
\hline Source & Victor H. Huynh \\
\hline Figure 56 & 3D Printed Aperture Experiment \\
\hline Source & Victor H. Huynh \\
\hline Figure 57 & Box Light Experiment \\
\hline Source & Victor H. Huynh \\
\hline Figure 58 & Reflected Coloured Light Experiment \\
\hline Source & Victor H. Huynh \\
\hline Figure 59 & Surface Manipulation \\
\hline Source & Victor H. Huynh \\
\hline Figure 60 & Aperture Experiment \\
\hline Source & Victor H. Huynh \\
\hline Figure 61 & Spatial Form Experiment \\
\hline Source & Victor H. Huynh \\
\hline Figure 62 & Screen and Light Filtration Experiment \\
\hline Source & Victor H. Huynh \\
\hline
\end{tabular}


$\begin{array}{cl}\text { Figure } 63 & \text { Physical Model - Site } \\ \text { Source } & \text { Victor H. Huynh }\end{array}$

Figure 64 Physical Model - Massing

Source Victor H. Huynh

Figure 65 Physical Model - Section Perspective

Source Victor H. Huynh

Figure 66 Physical Model - Detail

Source Victor H. Huynh

Figure 67 Day/Night Transformation - Dundas St.

Source Victor H. Huynh

Figure 68 Day/Night Transformation - Rusholme Rd.

Source Victor H. Huynh 

LIGHT AND SHADOW EXPRESSION:

Contrast, Luminosity, Porosity 

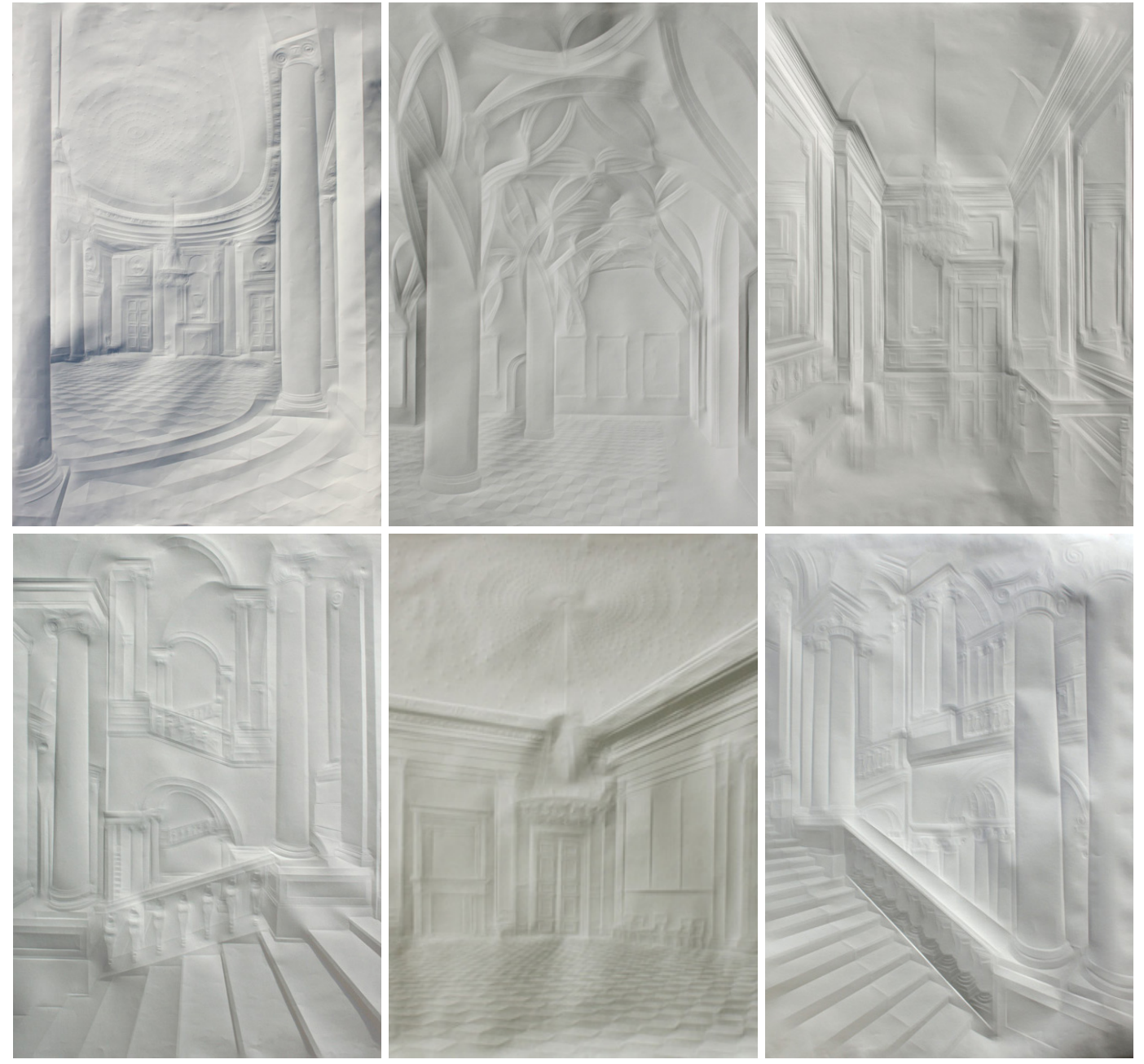

- F00

Paper Works by Simon Schebert

Folds in the surface that create a relief of just a few millimeters, catching the light at different angles to create detailed pictures of architectural spaces. 


\section{Introduction}

What is light? What is its significance to the human body, perception, experience and to architecture? Is it an artistic design expression that inspires the human spirit or is it a technical tool that provides the planet with energy and enable living organisms to see? The evaluation of architectural lighting in contemporary architecture has been increasing following the trend of quantifiable design reasoning, neglecting the importance of qualitative lighting design in shaping architectural environment and experience. (Rockcastle, Andersen 2013).

Throughout history, social, cultural and technological trends have influenced the integration and expression of light within architecture, changing the way user experience and perceive the built environment. Understanding the development and application of architectural lighting design throughout history provides insight of lighting design expression and experience within the built environment. Programmatic need and the architectural experience correlates to the type of lighting design integration and expression within architecture. Creative lighting design expression provides perceptual stimulation that enriches the architectural environment and user experience as environments perceptually transform throughout the day. Light influences the characteristics and perception of form, material, scale, colour, texture, and spatial ambiance. 
The development of modern design methods and technology introduced new approaches to illuminate space, changing the design of architectural environment and the way user experience and perceive space.

The saturation of light through modern glazing and artificial illumination reduced the presence of shadow and darkness within architecture. Modern glass allowed for larger windows spans, flooding the interior environment with light during the day as well as providing indoor/outdoor connectivity. Whereas artificial illumination provided architecture with a reliable source of spatial illumination in the absence of the sun.

The saturation of light within contemporary architecture has deprived users of the potential experience through the interplay of light and shadow. It is necessary to ask the question how much light does man need, as both light and shadow have a significant role in perception and experience. The integration of both daylight and artificial illumination has significant role in shaping contemporary architecture, redefining the way user experience and perceive the built environment throughout the day.

Creative lighting design expression within architecture examines the characteristics and quality of light a space receives. The analysis of different spatial lighting conditions provide insight on the possible lighting effects and its influence within architectural environments. The human 
eye is constantly adapting and receives various lighting stimulus that provide unique sensorial experience. Lighting variation and diversity within architecture create opportunity to enrich the architectural environment and introduce layers of experiential moment as the lighting condition changes throughout the day. The traditional office working environment has produced uniform light distribution and furniture layout, depriving spatial stimulus through

This thesis advocates the importance of expressing light and shadow as a design medium to shape architectural environments. The design strategies expressed correlates to the effects of light on user perception and experience within an office hub cater to small start-up businesses. The integration of light as a qualitative design expression examines its impact on the human body, architectural experience and the built environment, challenging the quantitative design evaluation of light that caters to function and efficiency. Design consideration of sunlight, artificial light, material properties, architectural form and spatial ambiance heightens the poetic relation between light, the built environment and its user. Through the creative integration and expression of light and shadow, architectural environments become perceptually fluid, transforming the user experience over the course of the day 
04 | Light and Shadow Expression 


\section{Chapter 1 - Discussing Light}

\section{Understanding Light}

Human beings are predominately-visual creatures; we receive most of our sensory stimulation through sight (Pallasmaa 2005). Light provides us with visual stimulation enabling us to experience and perceive the world around us. While darkness reduces our ability to see, heightening the use of our other senses can help us understand ours surroundings. In the book The Eyes of the Skin, Juhani Pallasmaa describes the importance of darkness and its influence on perception and experience. "Deep shadows and darkness are essential, because they dim the sharpness of vision, make depth and distance, and invite unconscious peripheral vision and tactile fantasy" (Pallasmaa 2005, pg. 46). Designing architectural environments that consist of dissimilar lighting scenarios provides different forms of sensorial stimulation that engage the user's eye, mind and body.

The analysis of light and its effects on the human body, perception and user experience offers creative design integration of both sunlight and artificial illumination within architecture. Light reveals the built environment to its users, while architecture has the potential to reveal the unique characteristics of light. Through the use of material properties and spatial ambiance, the type of lighting a space receives is able to perceptually transform the characteristic and experience of architecture. The interplay of light and shadow 

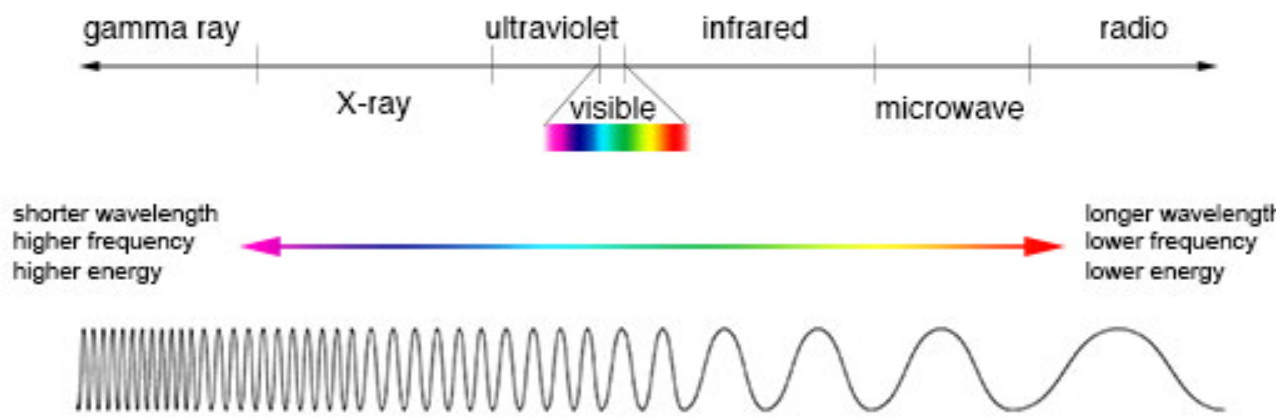

within architecture provides user with sensorial stimulation that change over throughout the day. The expression of light as a creative design medium considers its effects on user perception, its integration within the built environment and on the architectural experience. The evaluation of architectural lighting design techniques through sensory observation and scientific experiments is essential in understanding the effects of light within architecture.

A key discovery in understanding the physical properties of light resulted from a scientific experiment conducted by Sir Isaac Newton in the 18th century. In the treatise Opticks: of the reflections, refractions, Inflections and Colours of Light, Newton reported his discovery that light was comprised of a spectrum of electromagnetic radiation. Using a single beam of sunlight that entered a triangular glass prism, his experiment revealed that white light was comprised of a band of multi-coloured light. It was later discovered that each band of coloured light has its own distinct characteristic that have different effects on the human body and perception. Newton's experiment isolated light into a single entity, but neglected its counterpart, darkness.

When light is absence darkness is present, both characteristics are essential for the perception
- F01

Electromagnetic Radiation

Electromagnetic Spectrum of Light 


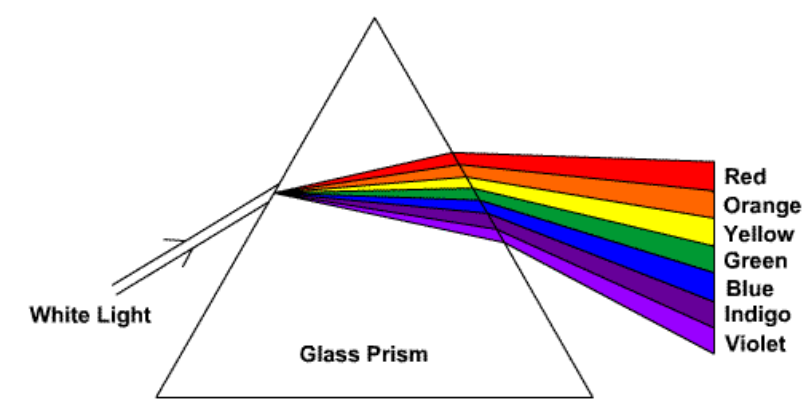

- F02

Light Prism Experiment Newton
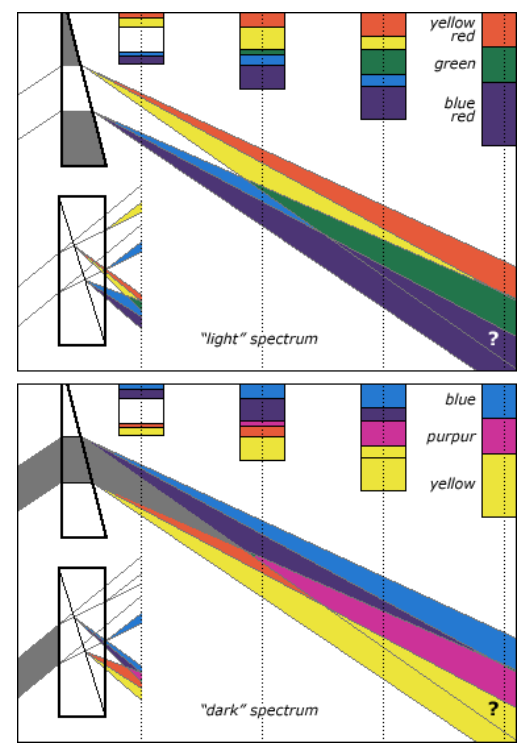

F03

Light Prism Experiment Goethe and experience. Johann Wolfgang van Goethe expanded on Newton's light prism experiment by experimenting with both light and shadow rays as they are essential to our daily lives. He concluded that colour was expressed between the boundaries of light and darkness. The crosssection of the refracted light varied the further it was from the prism. The expression of both light and shadow is important for perception of the world, and is necessary in the design of architectural environments and for the creation of related experiences.

The application of light within architecture can be categorize into two streams: quantifiable metrics and qualitative design expression. Quantifiable metrics take a scientific approach in the integration of light, examining it through energy and required illumination for task performance. Whereas the qualitative design expression examines lights experiential characteristics and its influence on the human body, perception, architectural environment and experience. The type of lighting expression within architecture varies accordingly to typology and programmatic needs. Where its use ranges from a functional requirement to an artistic and poetic integration within architectural environments. The reduction to architectural lighting as a method to illuminate space (commonly seen in the design of offices, warehouses, retail buildings, etc.) deprives 

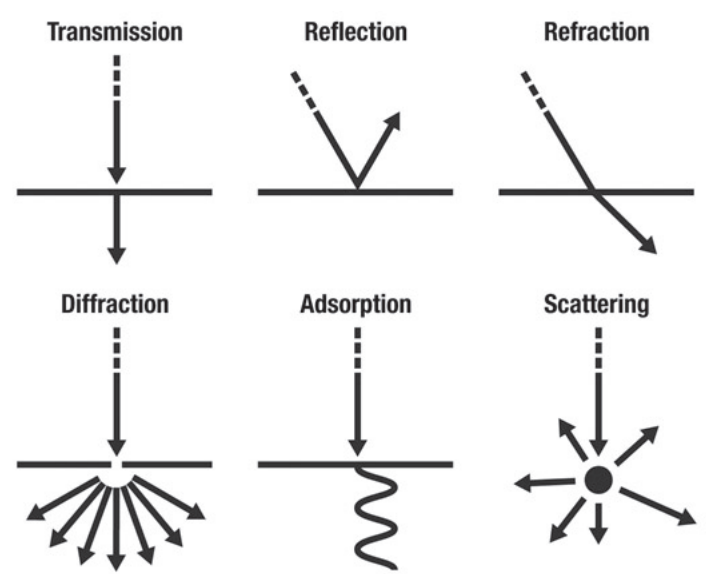

users of its dynamic characteristics to shape architectural environments (creatively expressed in the design of museums, galleries and religious buildings). It is essential to consider both spectra in the design of architectural lighting, ensuring a creative design expression while staying mindful of its functional requirements. The type of lighting a space receives is able to influence the occupant's comfort, enrich the architectural environment and experience (Meek 2015). The integration of light is a powerful design tool that requires the collaboration of multiple disciplines and design considerations (Descottes 2011). The expression light and shadow as a design medium has a significant role to in shaping architectural environment, one that can push the boundary of conventional thought.

Light itself is not visible to the naked eye and manifests itself through its contact with material. It is through this interaction that allows people to perceive and experience the surrounding environment. Louis Kahn describes material as "light that has spent itself" (Lobell, Kahn 1979, pg. 64). Shadows are a result of light not being able to transmit through a material, creating a dark void in its place. As light hits the surface of a material, the reflected light provides visual stimulation that enables us to distinguish its characteristics. Qualities such as colour, texture, form, and shape are revealed through light. The amount of light that
- F04

Light's Behaviour

The type of lighting effects experience vary between the material properties 

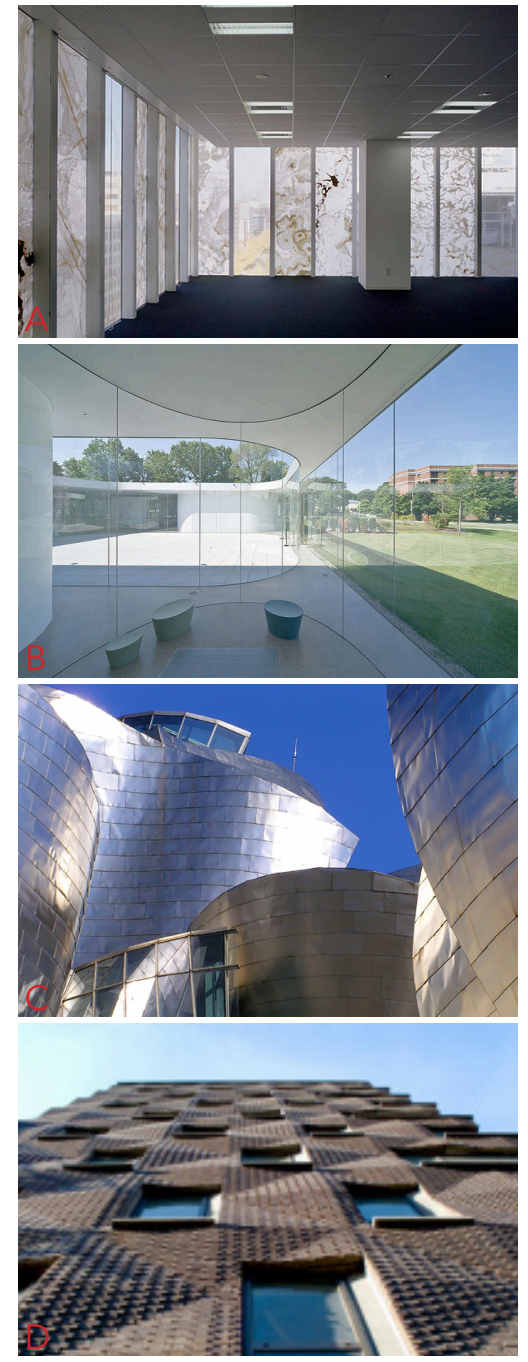

- F05

Material Characteristics
A - Translucent
B - Transparent
C - Reflective
D - Opaque

is absorbed, reflected, refracted, and transmitted through a material describe the characteristics of a material and can be categorized as being opaque, transparent, translucent or reflective material. Understanding the physical properties of material and its behavior with light provide potential design opportunities to experience and reveal the unique characteristics of light within architecture.

Opaque materials appear solid, restricting the movement of light through the surface. These materials reflect and absorb light, creating a spatial boundary and producing shadows as light is stopped at the surface. Transparent materials allow light to transmit through the material, providing a visual connection between spaces. Translucent materials behave similarly to opaque and transparent ones, allowing for emission of light even while some is absorbed and reflected. Their characteristics change based on the direction of the light source and on the overall lighting ambiance of the surrounding environment. Objects that appear solid in the morning can perceptually transform into illuminated objects at night through the use of artificial illumination. Lastly, reflective materials allow light to bounce off their surfaces, mirroring the colours and shape of the surrounding environment. Their unique properties allow them to perceptually transform throughout the day as they reflect and absorb varying amounts of light.

The quality and quantity of light is constantly changing throughout the day, influencing the perception and the appearance of material (Major, Speirs 2005). It is important to examine material properties under varying daylight conditions, since knowing their range is important to understanding the lighting effects produced (Steane 2011). Some materials appear to liven up under direct light while they appear dull when illuminated by ambient light. "Materials are critical to deepen our experience of light, to reveal what is unique to a site through the experience of light, as light's interaction with the material world largely defines one's interpretation of place" (Schröpfer, Carpenter 2011, pg. 117). 


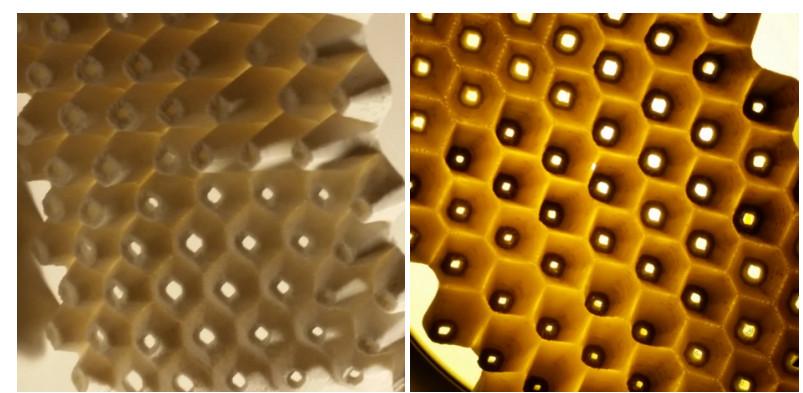

The advent of modern design technology has allowed for new construction methods that offer new design possibility with the use of traditional materials. An example of this is the use of stones in the construction of a wall. The stacking of stone blocks and the use of motor joints is described as the traditional method of construction that have been used throughout history to build walls. A stonewall is describe as being heavy and solid, and restricting the movement of light. Seen in the design of Dominus Wintery by Herzog de Meron, contemporary design method has allowed the stone wall to appear porous and filter light. A steel mesh cages the stone aggregates creating openings to display light and shadow effects along the ground. The wall behaves as a mediator between spaces, perceptually transforming throughout the day.

\section{- F06}

Material and Light Experiment The exploration of light on translucent material

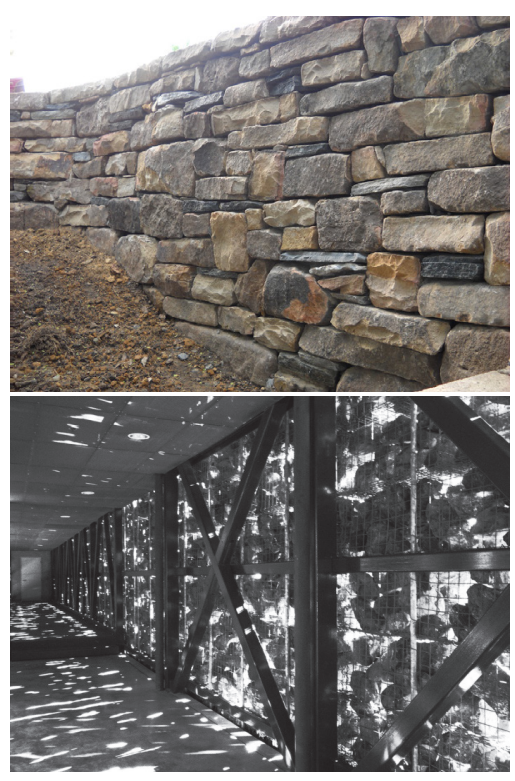

- F07

Stone Wall Construction Material assembly effecting the lighting characteristics of a wall 


\section{The Human Body, Perception and Experience}

It is an established fact that sunlight is an essential element for the creation and sustenance of life on the planet. Without it, life could not exist. The human body has naturally evolved according to the day and night cycle of the sun. After food and water, sunlight is the most crucial natural factor for the control of bodily functions (Mott et al., 2012). The human body has developed an internal clock known as the circadian rhythm that regulates the operation, distribution and regulation of hormones for optimization of energy and growth over the course of a day (Mott et al., 2012).

Research has shown that different colours light of the electromagnetic spectrum influence the way users perceive and experience the world them. Blue light has been shown to secrete the production of melatonin, and thus enables the body to feel more alert and awake. Understanding the different effects of light on the human body provides design opportunities to implement and improve the architectural environment. For instance, blue light can be integrated in working environments that require high productivity and alertness during the evening or morning. It can also be reduced in the evening in order to support the sleep cycle. The articulation and expression of the different types of lighting effects and condition within architecture enables the built environment to support and enhance user experience and overall well-being. 


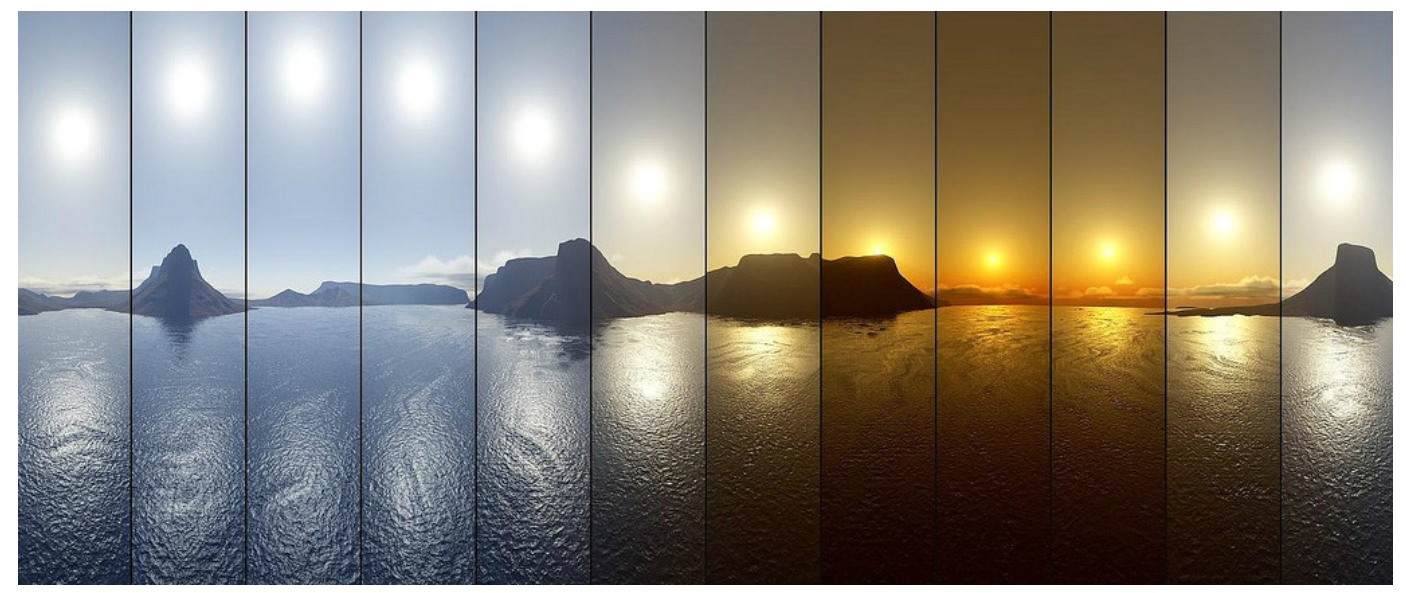

The rotation of the Earth along its axis produces a day and night cycle that changes the quantity and quality of light within the natural environment. The angular positioning of the sun changes the characteristics and perception of sunlight as it scatters within the Earth's atmosphere. A reddish-orange (long wavelength) hue of light is experienced during sunrise and sunset as blue light (short wavelength) scatters in the sky. During the afternoon, sunlight appears as white with its highest intensity. The sun is constantly changing throughout the day altering the way people experience and perceive the built environment. Light offers the potential to create perceptually fluid environments as its ability to transform the characteristics of material, space and experience. Using solar patterns as a design medium offers the built environment to express and reveal light a specific moments in time, creating dynamic environments for users to experience. Daylight is a phenomenon experienced in the interior environment, whereas artificial light is experienced in the exterior. Day to night, sunlight to artificial light: new experiential opportunities and activities are offered through these changing lighting conditions.

Artificial lighting cannot replace sunlight within architecture. The presence of sunlight within

- F08

Daylight Transformation

The dynamic characteristics of sunlight throughout the day 


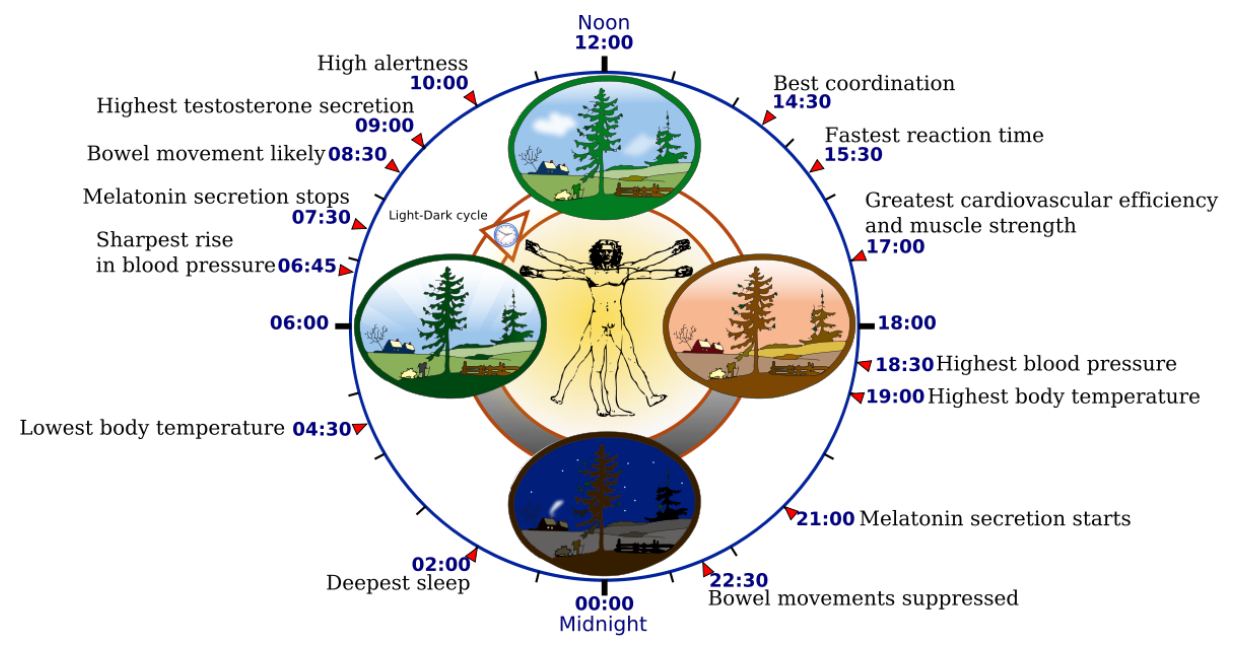

F09

Circadian Rhythm

An internal clock that

responds to light, regulating

bodily performance for

optimization and efficiency.

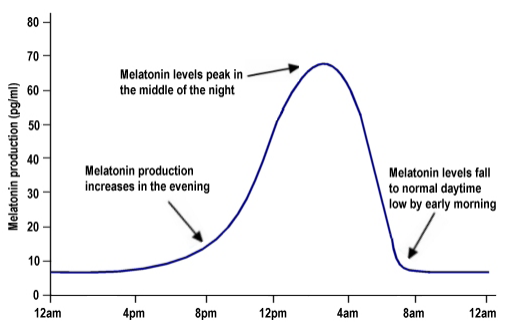

- F10

Melatonin Levels

The amount of melatonin (sleep induce chemical) in the human body throughout the day spaces has been proven to improve the overall performance, productivity and comfort of users (Meek 2015). Light "provide[s] the mental and visual stimulation necessary for the proper regulation of circadian rhythms" (Meek 2015, pg. 1). Design integration of daylight can drastically improve people's visual and biological experiences (Boubekri 2014).

"The visual environment relates to the proper conditions for good visual perception, comfort, and performance - important design elements in workplace environments. The biological environment represents the non-visual effects of daylight, such as those relating to the production of the serotonin and melatonin hormones that regulate our vital circadian rhythm"

(Boubekri 2014, pg. 11).

The lifestyle of modern society has extended working hours late into the night while being overly exposed to artificial illumination and the lack of daylight exposure, negatively impacting the human body's performance (Boubekri 2011).

The increase concern of economic and sustainable design criteria has value the design of quantifiable design metrics of light, the evaluation of lighting 
design through energy and minimum requirement for spatial illumination. This emphasis has devalued the qualitative approach to application of light and has deflected concern with the psychological and biological well-being of users (Schoof n.d.). The implementation of architectural lighting requires an understanding of both its perceptual design impact on the human body and experience, and its technical application and economic demands. Architectural lighting design through codes and standards (metrics) have create homogeneous environments that deprive user of perceptual and visual stimulation. In the 21 st century, the progression of architectural lighting from a field of engineering towards a creative design discipline is being examined and explored by architects (Karcher 2009).

Light as a qualitative design expression is seen in the works of Steven Holl. His architectural projects integrates light in relation to user perceptions and experiences, creating perceptually fluid and dynamic environments in correlate to sunlight and artificial illumination, transforming the day-to-day experience. The expression of light as a creative design medium offers a powerful design tool that can enrich the overall experience of architecture.

Light has important role in experience and sight as described by Juhani Pallasmaa, "I exist in life only if I can see" (Pallasmaa 2005, pg. 27). Human beings are predominately visual creatures, we receive about $80 \%$ of our sensory information regarding the world through our eyes (Karcher 2009). Light provides us with visual stimulation that enables us to perceive the world. Our understanding of the world is through what we see and vision by Plato as humanity's greatest gift (Pallasmaa 2005, pg. 15). Light provides us with the ability to see, and thus interpret the world around us (Pallasama). Without light, the characteristics of material and space diminish; we are unable to capture details such as material, shape, form, texture and colour. 

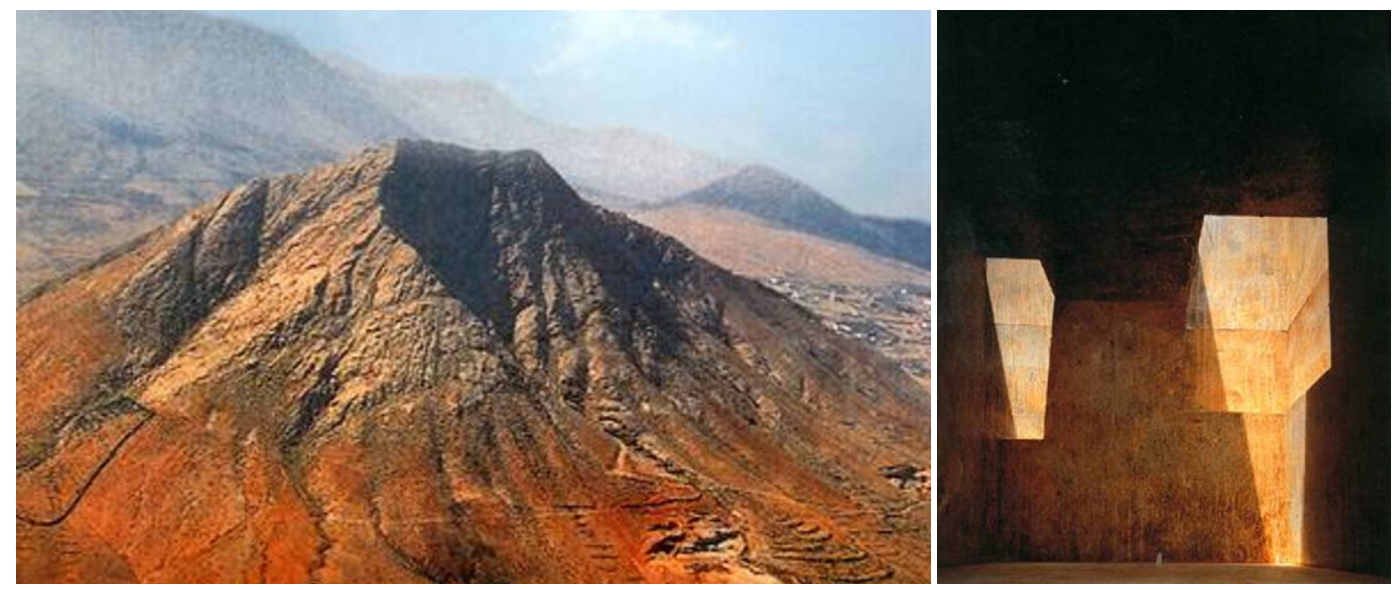

F11

Tindaya - Eduardo Chillida

Sculptural project exploring light and perception
The absence of light reduces our ability to perceive objects and space clearly, thus altering our perception and understanding of the world. Henny Larsen vividly illustrates the importance of light and vision in the perception of space:

"We step inside a dark cave. With no light in the cave, we cannot form any conception of it. Were you to describe the cave by running your hands over every inch of it, ceiling, floor and walls, it would take several lifetimes to gain any sense of how the cave looks. But the moment you strike a light you see the cave as a room, instantly perceiving it as a space. Space is a much more complex concept than form, and only much later in life do we learn to relate to it."

Henny Larsen

(Major, Speirs 2005, pg. 25)

Light is intangible to the naked eye and reveals itself as it interacts with material. This is what allows the eye and mind to process information and experience the world around us (Major, Speirs 2005). Understanding the process of the way people perceive space in relation to the movement of light provides an alternative means of designing and integrating light within architecture. Light is a photon of energy traveling through space, and as it 

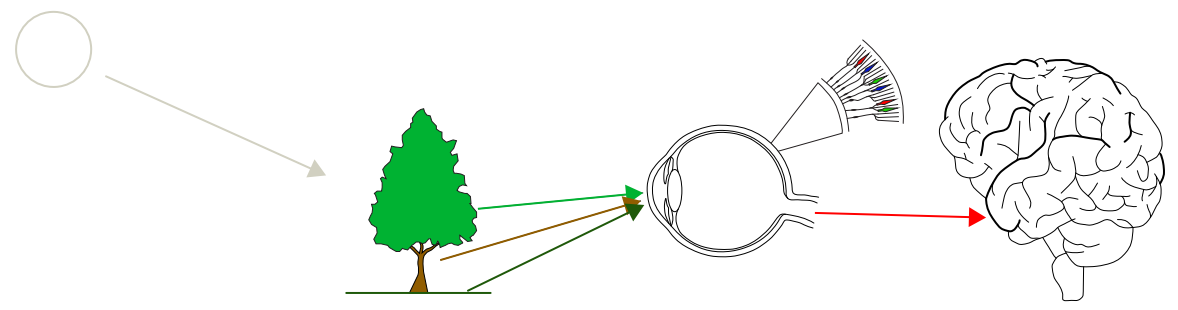

The Light Source

Material Interaction

Visual Stimulus

Processing

contacts a surface it provides visual stimulation for the eye to perceive. According to the properties of the material, it absorbs, reflects, refracts or transmits light, revealing such characteristics as colour, texture, shape, and form.

The amount of light that interacts with a material alters our understanding and perception of that material. Light that is reflected of the surface is absorbed by the retina of the eye, enabling people to perceive the material. Light provides the human body with visual stimulation that is then processed and stored within the brain. "When signals are sent from our eyes to our brains, the process of interpretation of such stimuli is called perception. 'Reality' therefore, is nothing more than the interpretation by the mind of the images focused on our retinas" (Major, Speirs 2005, pg. 79). Working in unison, our eyes and brain are constantly adapting and responding to the surrounding environment, seeking stimulation through light variation.

Henry Plummer states that the "quality of light is of paramount importance" within architectural spaces (Plummer 2009, pg. 13). "The correct illumination intensifies the poetic and emotional impact of a project" (Verges 2008, pg. 9). Whether in broad daylight or dim light, our eyes are capable of perceiving space under a variety of different lighting scenarios, providing different sensory
- F12

Understanding Perception

Diagram of the process of

light and perception 


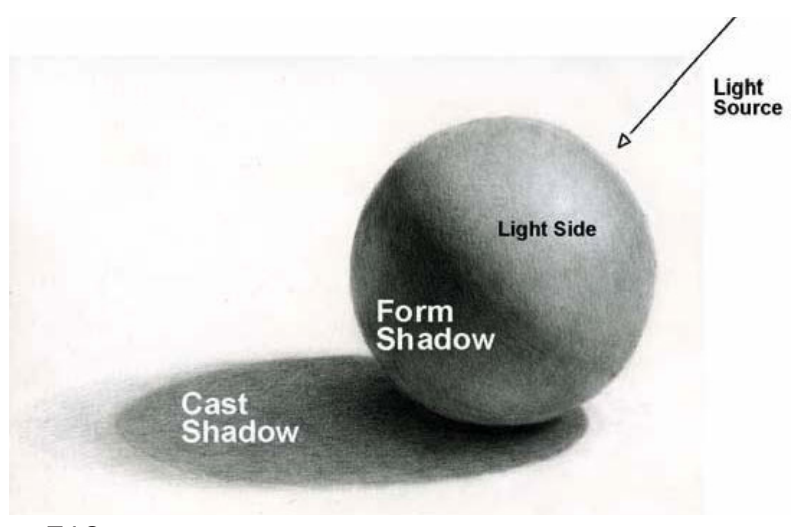

F13

Light \& Shadow Revealing Form

stimulation and experiences (Major, Speirs 2005).

In the book Environmental Diversity in Architecture, Mary Ann Steane and Koen Steemers describe the importance of designing non-homogeneous environments in order to improve the overall architectural experience, as well as its physiological and psychological well-being of the user. The eye's built in adjustable sensors provide users with different visual lighting stimulation, such that the lighting experience under one set of circumstance will produce a different sensation under another set of circumstances (Steane, Steemer 2004, pg. 181). The surrounding environment and the overall experience throughout space attributes to the overall lighting experience. Working simultaneously, the eye and the brain distinguish subtle changes in lighting conditions that help us understand depth and complexity (Liljefors 1997).

Uniform spatial illumination and light saturation have negative effects within architectural environment, both depriving users of the potential experience through lighting variability and the interplay of light and shadow. Lighting variation is beneficial in creating visually diverse and stimulating environments, ones that engage the eye, mind and body (Rockcastle Andersen 2013). The eye seeks information manifested by contrast, distinguishing the difference between brightness and/or colour 


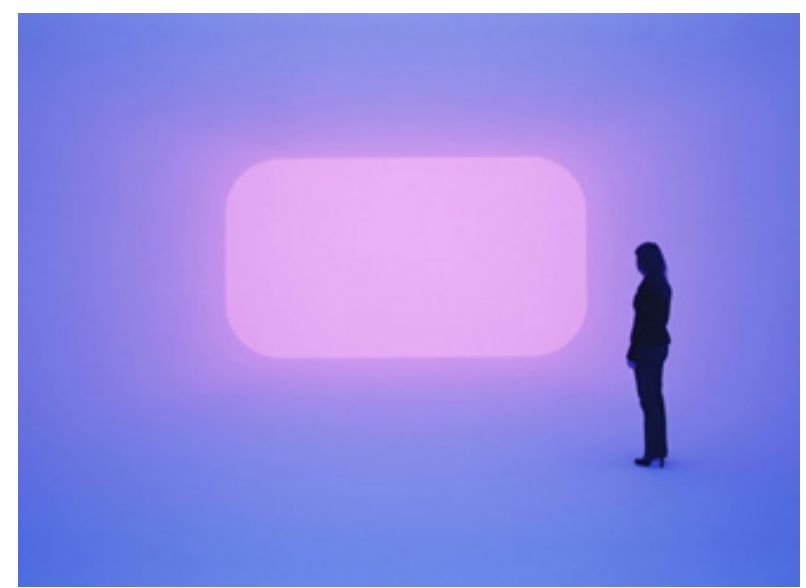

(Liljefors 1997, diversity 189). Pallasmaa writes that "The human eye is most perfectly tuned for twilight rather than bright daylight" (Pallasmaa 2005, pg. 46). The saturation of light within contemporary architecture has deprived users of the sensorial experiential opportunity produced by low spatial lighting ambiance and darkness. These reveal the unique characteristics of material, shape, texture and form.

Known as the Ganzfeld effect, the exposure to unstructured uniform stimulation fields results in the brain amplifying neural noise in order to provide missing visual signals. This is commonly seen in architectural environments designed through codes and standards to maximize function and efficiency as it provide equal distribution of light through a grid layout. This neglects the potential of lighting variability to provide visual stimulation that engages the eye, mind and body. "The more diverse the luminance in the field of view, the more 'pleasant', 'cheerful', 'bright', 'radiant', 'clear', 'visually warm' and 'strong' the space to appears" (Steane, Steemers 2004 pg. 190). James Turrel, an artist who express light as a creative design medium within his projects utilize light as a medium to alter spatial perception and experience.

The re-evaluation of light within contemporary architecture is necessary, addressing both its
- F14

Ganzfeld - James Turrel

Distortion of perception, reality and space through back-lit artificial illumination. 
technical and experiential design expressions in shaping an architectural environment and spatial experience. (Rockcastle, Andersen 2013, pg. 20). Marilyne Andersen, a member of the Interdisciplinary Laboratory of Performance Integrated Design at EPFL (École Polytechnique Fédérale de Lausanne) advocates the importance of daylight integration within architecture as it has beneficial effects on the health, well-being, performance and experience of building users. Andersen elaborates on the importance designing space through perception and experience rather than codes and standards: "Perceptual qualities such as contrast and temporal variability are essential to our appreciation of architectural space; natural illumination adds depths to complex geometries and infuses otherwise static interior spaces with shifting compositions of light and shadow" (Rockcastle, Andersen 2013, pg. 19). The ever-changing lighting conditions throughout the day offers architects a dynamic design element, providing new experiential opportunities through the expression of both sunlight and artificial light. 
20 | Light and Shadow Expression 


\section{Chapter 2 Contrast}

\subsection{Historical Application}

Throughout years of human civilization, methods of expressing light in the built environment have evolved in relation to social, cultural and technological changes. Understanding light's architectural development throughout history provides insight into design integration within contemporary architecture (Brogan 1997).

The sun is the primary source of illumination and is consider as the giver of life on the planet. Solar patterns determine day to day activities and influence the way people experience and interact with the built environment. At the start of human civilization, the expression of light within architecture had a metaphysical and divine aspect that affected the way people perceived and experienced spatial environments.

The planning and design of the built environment revolved around solar patterns. Architectural environments that were highly experiential evolved as spatial environments changed over time. Temples, pyramids and ancient cities employed light as a method to perceptually stimulate and inspire the human spirit. Symbols, icons and selective design elements predominated through the use of light for worship. The employment of light and shadow within the interior of religious and monumental architecture influence user 


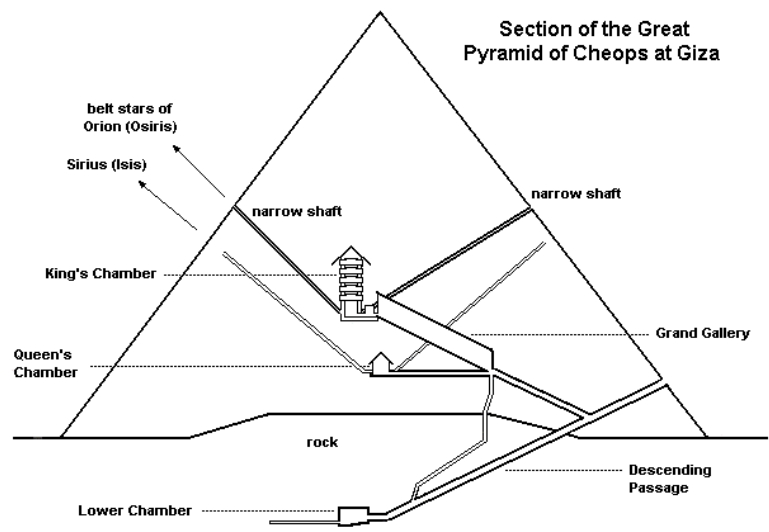

perception and experience. A powerful component of design, light influenced spatial conception, organization, scale, orientation, material selection and composition, architectural form, and many other aspects. The metaphysical expression of sunlight was an attempt to understand the world and life itself. Celestial bodies of light in the sky provided means of organizing and orientating ancient architectural projects such as ziggurats, pyramids, shrines, churches and temples.

The construction methods of their eras resulted in massive load-bearing walls and dark interior environments with small windows. Structural limitations created design restrictions on, for instance, the amount of building openings and light that could enter through them. Allowing for taller structures with large windows requires walls to have greater depth in order to support the additional weight of the design. Massive walls created a threshold as light mediated between the exterior and interior environment. A gradation of lighting conditions was notable as light washed along the surfaces around a window, creating a range of perceptual lighting stimuli.

Architects of the past understood the value of utilizing contrast as a means of designing environments that contained a variety of lighting experiences and stimuli. The presence of light was
- F15

Pyramid of Giza

Celestrial bodies defining aperture location

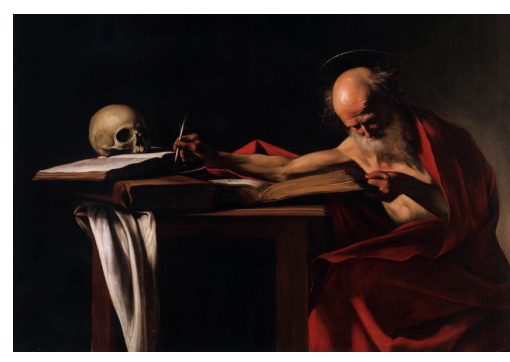

- F16

Chiaroscuro

Light and shadow expression in art as a method to create dramatic stories 

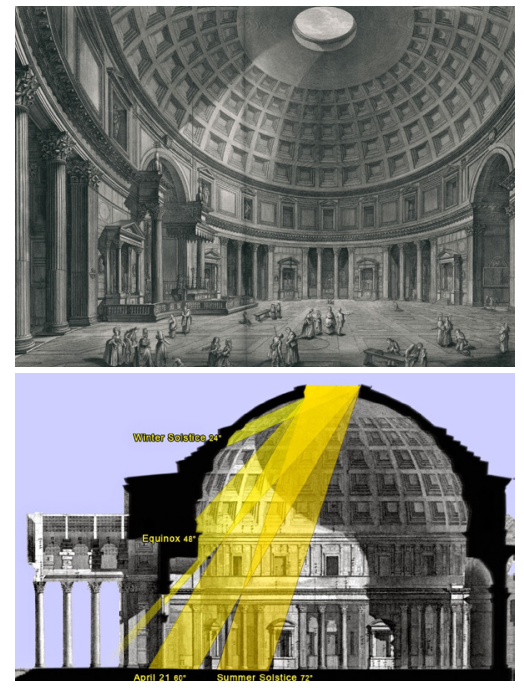

- F17

Pantheon

A single oculus illuminating space often dramatic because of its contrast with its dark milieu. Darkness was express as a design method to desensitize the body form visual stimulation, creating calming and ambiguous environments conducive to contemplation. The contrast of light and dark effectively revealed such architectural features as colour, texture, shape, form and material.

As a result of new design philosophies, construction methods, and social and cultural changes, new lighting design expressions were introduced that transformed environments as well as users' experience of them. Throughout the Roman Empire, light was used in its fundamental sense to highlight design elements and illuminate space. The quantity and quality of light as it changed throughout the day altered the characteristics of both environment and experience.

The Pantheon is one significant project that integrated sunlight to generate a distinctive architectural environment. It utilized solar patterns as a method to highlight specific figures and design details, thereby generating a distinctive ambiance. (Brogan 1997). The size and scale of the building were designed in relation to one another, ensuring enough illumination for spatial functioning. A single oculus was used to illuminate the interior environment, letting in sunlight that came into contact with the interior floor, walls and ceiling. Various interior ornamentations and detailing produced light and shadow effects that enriched the architectural experience. The Pantheon, in a manner similar to a sundial, provided a single beam of light to measure time, thereby creating experiences that were particular to a specific moment in time.

During the Gothic period, light was used as a communicative design medium that depicted stories for users to perceive and experience. The development of the structural buttress provided relief for exterior walls, allowing buildings to be taller, and to have thinner walls, more openings, 
and larger windows. More light was introduced within interior environments, creating experiential opportunities that were new in comparison with Roman architecture. The use of stained glass windows artistically and poetically displayed light to its users, creating ambiance within churches as it vividly displayed colours and light within interiors (Ramos 2015). The expression of light possessed a sacred and religious connotation, it encouraged belief and evoked emotion as it penetrated translucent materials. Spiritual rebirth was symbolized by light in churches as dark interiors were illuminated by stained glass windows.

Architectural lighting during the Renaissance expressed a soft ambiance that highlighted design characteristics and detailing within materials and forms. Calm, uniform lighting distribution provided for visual clarity of the shape of an object. In the Ecstasy of Saint Teresa, the built environment was designed to support the illumination of the sculpture. Through the use of a north-facing skylight that was hidden from viewers, uniform spatial illumination gave clarity to highly sculptural detailing and characteristics of materials. White marble was used to sculpt the figure, allowing high contrast of light and shadow conditions as light reflects off this material. Gold foil was used on the sun rays of the sculpture, creating a highly reflective design element that created indirect illumination throughout the day. As design principles change over time, understanding the significance of light and shadow as a design expression impacts the conception of architectural environments especially as they relate to user experience and perception.
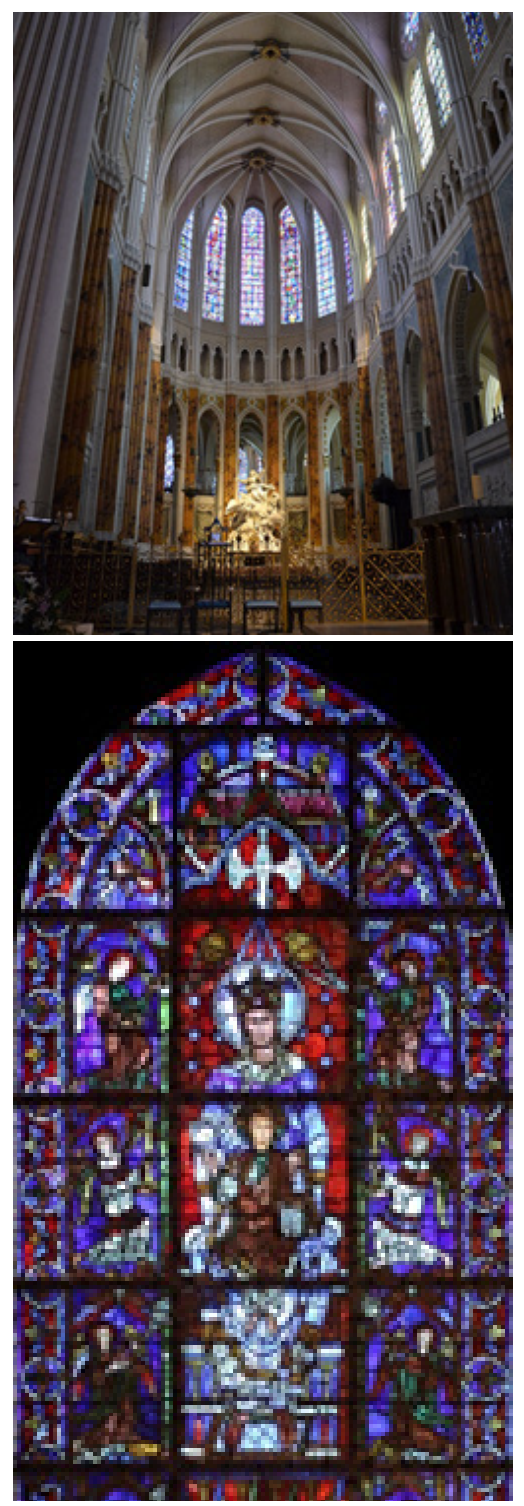

- F18

Stained Glass Window

Symbolic representation of

light through colour and stories 


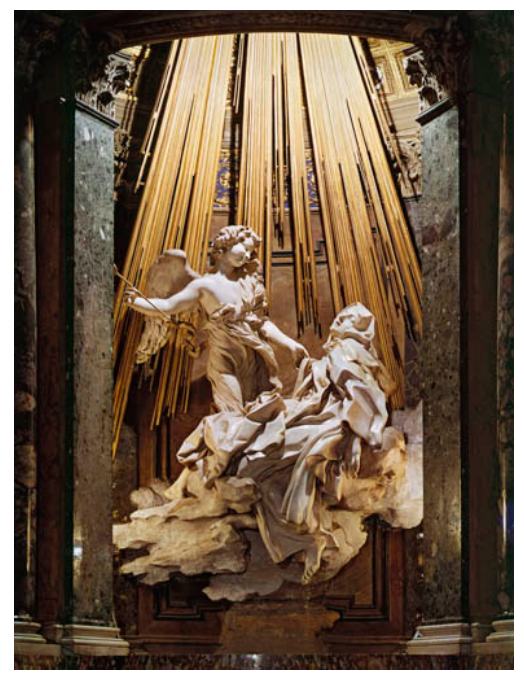

- F19

Ecstasy of Saint Teresa

Material properties revealed through the use of soft spatial illumination
"If light is scarce then light is scarce; we will immerse ourselves in the darkness and there discover its own particular beauty"

Jun'ichir冈 Tanizaki

(Tanizaki 1977, pg. 31)

\section{In Praise of the Shadow}

Technological transformations, social and cultural changes and new construction methods during the Industrial Revolution transformed architectural lighting expression. Modern design philosophy changed the expression and integration of light within architecture, favouring concepts pertaining to luminosity, visual clarity, function, efficiency and engineered aesthetics. However, the trend towards saturation of light within architecture neglects the value of darkness and shadow as a design medium. The pervasive use of glass and artificial illumination has grown as a design trend within contemporary architecture, although some modern architects understand the value of darkness in structuring light within spatial environments. Architects such as Tado Ando, Louis Khan, Alvar Alto, Peter Zumthor and Le Corbusier expressed light and shadow as a method to shape architectural environments and spatial experiences within their designs. Designing light in relation to perception concedes the significance of utilizing different lighting conditions and acknowledges what they offer to the human body and human spatial experience.

Written in 1933 by Junichiro Tzatziki, the literary essay "In Praise of the Shadow" critiques the issues of modern architectural lighting design through the analysis and comparison of traditional Japanese architecture and culture. Tanzaiki states that the expression of light and shadow within Japanese 


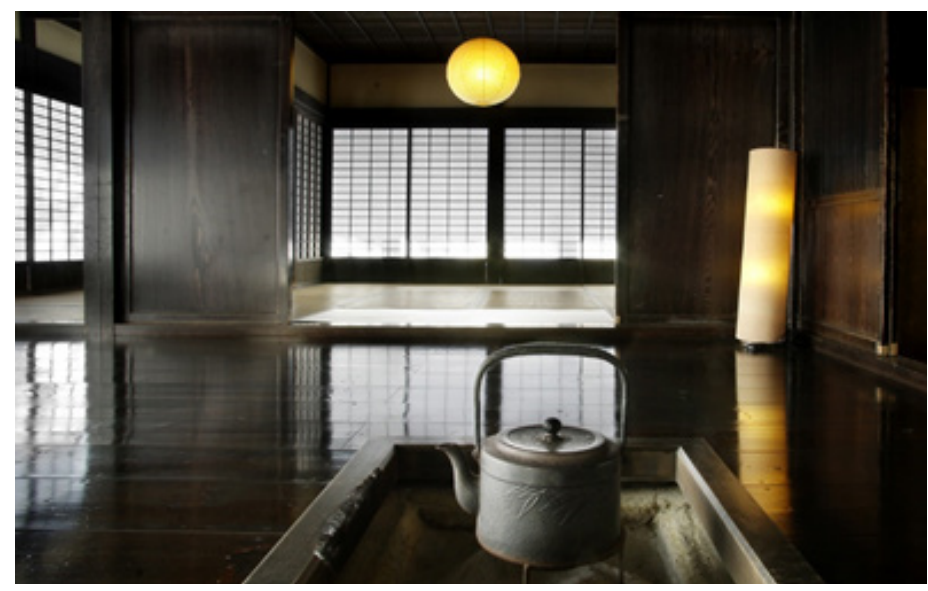

architecture was a response to the lifestyle of our ancestor and traditions that have developed over time.

"The quality that we call beauty, however, must always grow from the realities of life, and our ancestors, forced to live in dark rooms, presently came to discover beauty in shadows, ultimately to guide shadows towards beauty's ends"

(Tanizaki 1977, pg. 18).

Local traditions, climate conditions, construction methods and design principles vary from location to location, influencing the integration and expression of light within architecture. Cities located away from the equator often desire sunlight within buildings as a method to passively heat and illuminate spaces. However, cities along the equator screen light in order to reduce excessive heat gain throughout the day. The integration of daylight itself provides a means of contextual design intervention that eliminates universality of modern glazed curtain walls (Frampton 2002).

The saturation of light through the use of modern glazing and artificial illumination has deprived architectural environments of the experiential potential of the interplay of light and shadow. "In a world where we can now create light on 


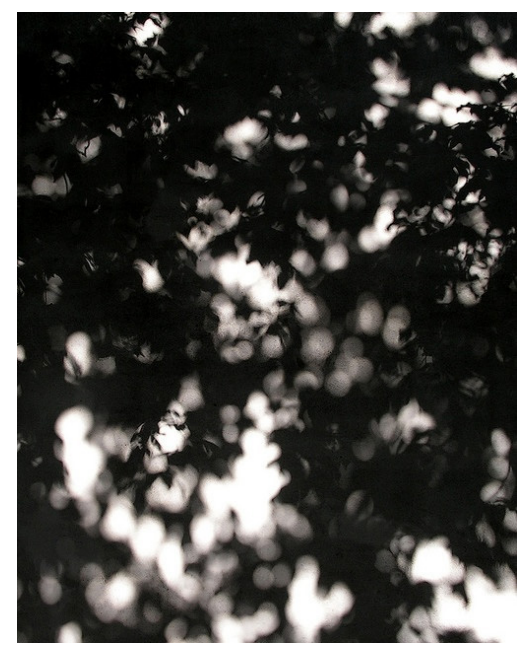

- F21

Light and Shadow Patterns

Perceptual beauty of variation

in light and shadow demand, darkness has found places where it is both necessary and welcome" (Major, Speirs 2005, pg. 62). Contemporary architecture has relied heavily on light as a means to provide visual clarity of objects and forms, whereas the use of darkness enriches the architectural experience by reducing the sharpness of our vision, necessitating the use of our other senses to perceive spatial environments. There is a range from high to low spatial lighting ambiance. The type of lighting spaces receive alters the characteristics of its materials and ambiance, as well as the activities within.

Tanizaki claims that the saturation of light in modern spatial environments has deprived users of the experiences and of the understanding of the unique properties of material in relation to light, "Modern man, in his well-lit house, knows nothing of the beauty of gold" (Tanizaki 1977 pg. 20). The high reflectivity of gold allows that material to constantly shine and reflect light under high or low spatial lighting condition. Gold's reflective characteristics allow it to be the primary focal point in any spatial lighting condition. It was used in religious sculptures to draw attention and as a means of providing indirect illumination in dim spatial environments.

Described as simple and refreshing, the articulation and expression of light and shadow within 
traditional Japanese houses has influenced their design features. (Tanizaki 1977). A dialog between the built form and the sun is expressed within designs; there are environments that filter, screen, block, shelter or enable direct sunlight to enter the building based on the time of day. Environments perceptually transform in relation to the type of lighting they receive; the use of light and shadow creates new experiential opportunities.

Tanizaki states that the experience and expression of light and shadow within architecture is far more important that the design of form: "We find beauty not in the thing itself but in the patterns of shadows, the light and the darkness, that one thing against another creates" (Tanizaki 1977, pg. 30). The interplay of light and shadow provides the built form with experiential depth as dark shadows overlap with light lines, and as the whole interaction is dynamic throughout the day.

In the design of Church of the Light (1989), Japanese architect Tado Ando utilizes both light and darkness in order to enrich and support the architectural experience. The church is designed to immerse users in an abyss of darkness, creating a calming and meditative milieu that desensitizes the human body from visual stimulation. Utilizing light as a metaphorical symbol for religious belief, the separation of the wall provides spatial illumination 


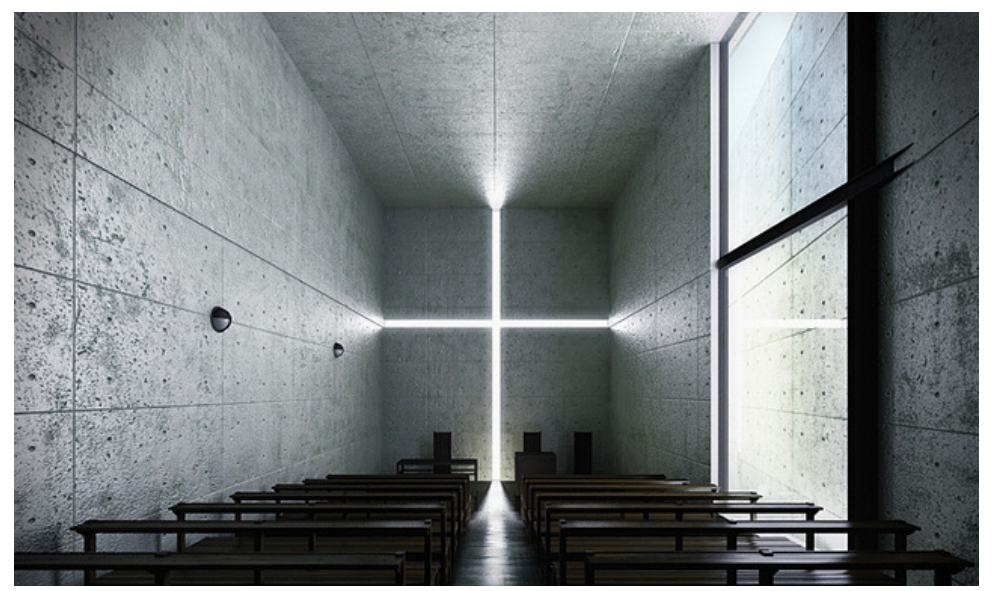

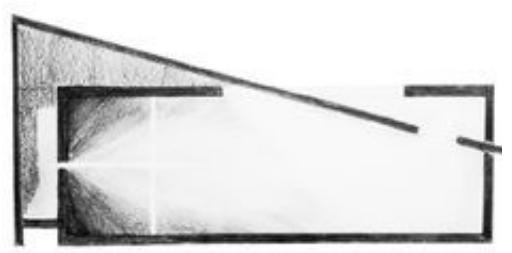

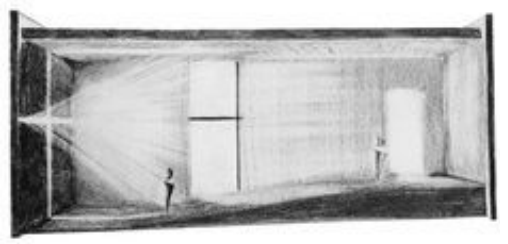

- F22

Church of the Light - Tado Ando The role of darkness in shaping light in the form of a cross. The small opening contrasts with the dark interior environment, providing a focal point and evoking spirituality. "The void itself becomes the raw material for knowing the possibility of being" (Richards 2006, pg. 9) Francesco Dal Co on Tadao Ando). A highly sensorial environment is perceived within the dark space, where the low light ambiance of the cross reveals the textures of the concrete walls and wood floor.

Ando integrated two different types of lighting methods within the design; the first one is described as light cutting sharply within darkness and the second one expresses light that is scattered and diffused within space. One acts as a focal point while the other creates spatial ambiance within the design. The church utilizes reflective light along a concrete wall to provide a soft ambiance to support the functioning of the church throughout the day. The church successfully expresses different spatial lighting methods that influence user experience and perception. By integrating different lighting conditions within environments, our eyes, mind and body are constantly adapting to the various lighting stimulus within, enriching the overall architectural experience (Boyce 1981).

In the words of Le Corbusier: "Architecture is the masterly, correct, and magnificent play of masses brought together in light. Our eyes are made to 


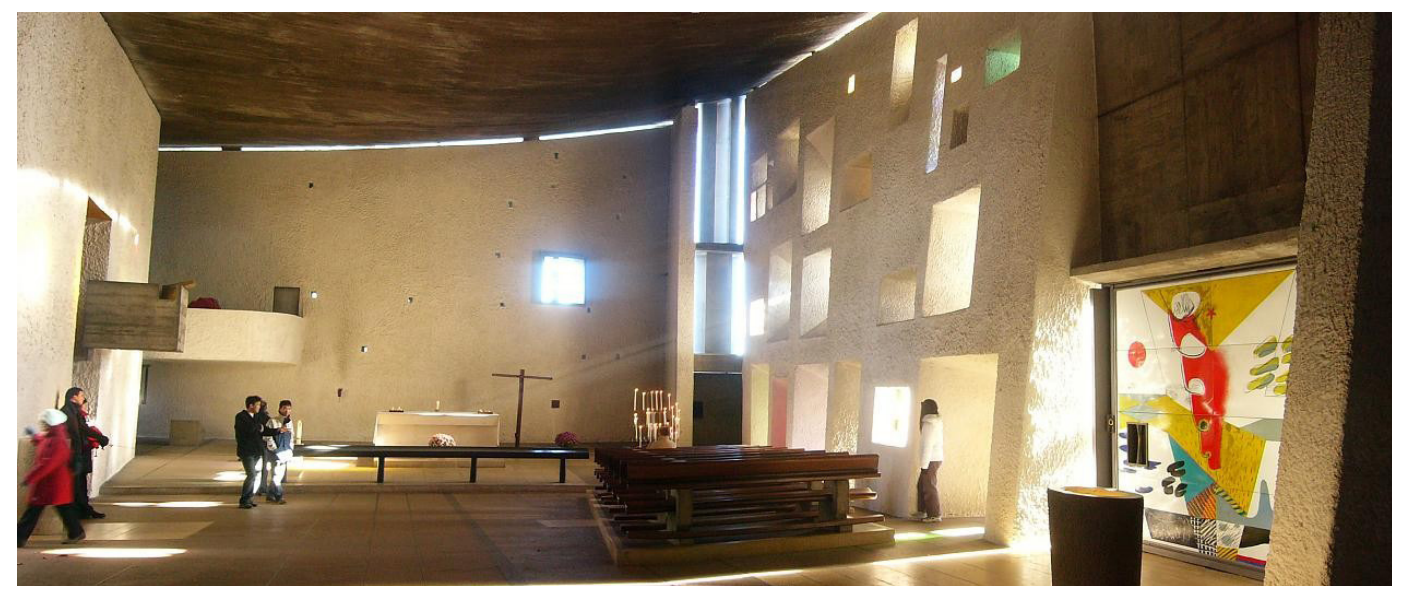

see forms in light; light and shade reveal these forms." (Corbusier 1986, pg. 29). Light reveals architecture and architecture reveals light. The integration of light within the built environment has the potential to change the way we perceive and experience space. One of the pioneers of modern architecture, Le Corbusier understood the value of darkness in structuring light later in his career. The articulation and expression of light and shadow in the Chapel of Ronchamp (1954) helped shape the architectural design. The employment of highly textured surfaces, colours, and the redirection and filtration of light provide enriching perceptual stimulation (Steane 2011).

Roofs, walls, openings and materials were express as optical instruments, providing a variety of different lighting effects that change in relation to solar orientation. The three design treatments that help support the expression of light and shadow within Ronchamp are: increasing the wall depth to allow light to mediate through; the filtration or screening of light; and the use of selective openings throughout the architectural form. Ronchamp is an example at which light and shadow is treated as a design medium to shape architectural environments and experience. The structuring of light through darkness amplifies the presence of light. Spatial lighting variation provides within the design provides engaging experiential opportunity

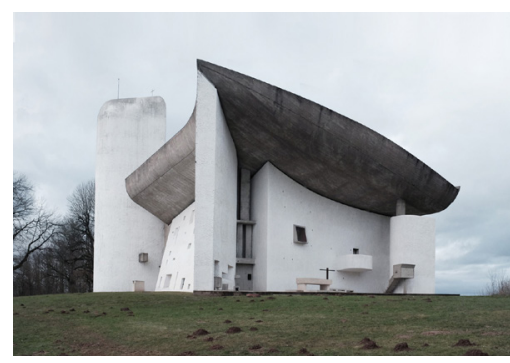

- F23

Ronchomp - Le Corbusier Light and darkness expressed through the architectural form, textured material, and colour 
that are unique to spatial function and time of day. Its perceptual transformation provide unique lighting experiences and moments throughout the day. 
32 | Light and Shadow Expression 


\section{Chapter 3 - Luminosity}

\section{Modern Transformation}

During the Industrial Revolution, the introduction of new technologies and design concepts, along with cultural changes, resulted in new design expressions that included innovative ways of expressing light. The traditional architecture of the 19th century and early 20th centuries has been described variously as machine aesthetics, showcasing the minimalist ideals, the expression of form, design clarity and luminosity (Riley 2003). The manufacturing process allowed for efficient means of mass production of material and products. Natural materials rich in texture and colour were replaced by panelized sheet material. Architecture represented timeless objects and presented designs that showcased the ideology of perfection through highly polished surfaces and forms.

Architecture changes constantly as new design methodologies and technologies are introduced. "Industry has created its tools. Business has modified its habits and customs. Construction has found new means. Architecture finds itself confronted with new laws". (Corbusier 1986, pg. 283). The expression of light, along with other aspects of design, has evolved in response to these new design ideologies.

Modern architecture utilized structural columns as a method to support the dead load of floors and roofs, eliminating the role of structural walls. This 
allowed for more openings in exterior walls, as they could now be designed lighter and thinner. An open floor plate concept allowed light to flow between spaces, creating highly illuminated interior environments. The façade plays a vital role in the experience and mediation of light as described by Louis Kahn,

"The great historical architectures were built in masonry, which necessitated thick walls. Openings in these walls reflected the light from their sides and sculpted the light as it entered the room. Modern architecture, with the sophistication of its thin materials, does not provide such modulation of light"

(Lobell, Kahn 1979, pg. 64).

Wall depth plays a vital role in the mediation of light between exteriors and interiors. Having a wall with a greater depth allows more opportunities to modulate and express light and shadow conditions in comparison to a flat or thin wall assembly. Modern lighting design expression values the use of reflectivity and glass, whereas historic architecture displayed a fascination with light and shadow effects within materials and the assembly as a whole in order to generate the architectural environment and experience (Richard 2006).

The saturation of light within architecture has deprived users of the potential experience of interplay of light and shadow. It is important to ask the question: "How much light does man need, and how much darkness?" (Verges 2008, pg. 10). Juhani Pallasmaa discusses the issue of light saturation in relation to architectural experience, "Homogeneous bright light paralyses the imagination in the same way that homogenization of space weakens the experience of being, and wipes away the sense of place" (Pallasmaa 2005, pg. 46). Lighting design through codes and standards produces homogeneous environments that maximize function and efficiency. Architectural environments can creatively integrate and express

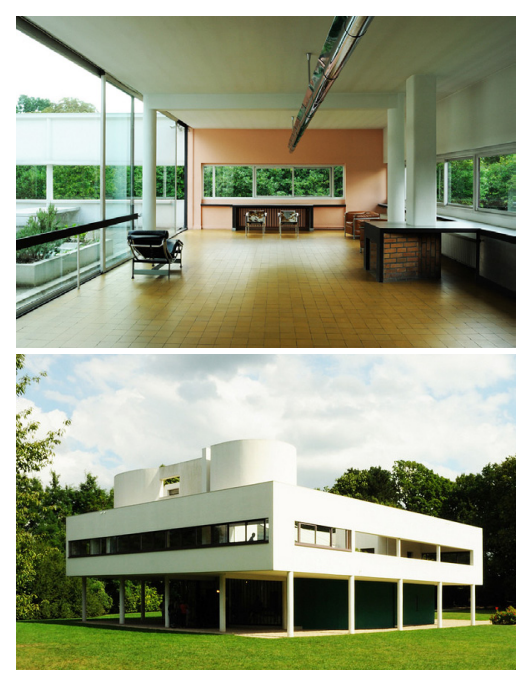

- F24

Villa Savoye - Le Corbusier Horizontal windows provide equal light distrbution within interior enviornments 
light in order to engage user perception while enriching the architectural experience.

The wide spread use of glass curtain walls in contemporary architecture has limited the understanding of different lighting experiences. Luis Barragan explains:

"The use of enormous plate windows... they deprive our buildings of intimacy, the effect of shadow and atmosphere. Architects all over the world have been mistaken in the proportions which they have assigned to large plate windows or spaces opening to the outside... we have lost our sense of intimate life, and have become forced to live public lives, essentially away from home

(Pallasmaa 2005, pg. 102).

Large glazed windows allow for a seamless connection between interior and exterior, creating highly illuminated interiors that connect with the outdoor environment. Increasing the amount of daylight within the interior is described as creating a more pleasant, cheerful and comfortable effect. Quality may exceed quantity, as Pallasmaa points out. "Most contemporary public spaces would become more enjoyable through a lower light intensity and its uneven distribution" (Pallasmaa 2005, pg. 46). The expression of light and shadow as a design medium examines the effects of different spatial lighting conditions on user perception and on the architectural experience.

Modern architecture replaced hand-crafted materials with manufactured components, reducing the interplay of light and shadow through impurities and imperfections. Pallasmaa explains the characteristics of materials after the Industrial Revolution. "The Modernist surface is treated as an abstracted boundary of volume, and has a conceptual rather than a sensory essence. These surfaces tend to remain mute, as shape and volume are given priority; form is vocal, whereas matter 
remains mute (Pallasmaa 2000, pg. 78)"

He continues: "Modernist architecture has preferred materials and surfaces that seek the effect of flatness, immaterial abstractness and timelessness" (Pallasmaa 2000, pg. 78). Natural materials have a non-uniform chemical composition; their variations in textures, colours, and densities produces irregular lighting patterns. These subtle variations are revealed under light, providing subtle perceptual stimulation that enriches the architectural environment.

Gaston Bachelard explains that Modern architecture has been preoccupied with formal design as the architectural experience, neglecting the importance of material expression and design application. "He considered that images arising from matter project deeper and more profound experiences than do images of forms. Matter evokes unconscious images and emotions, but modernity at large has been primarily concerned with form" (Pallasmaa 2000, pg. 80). Thomas Schroepfer expresses his desire that architecture not conform to traditional ideas, but rather celebrate materials and reveal them through light, expressing them in a manner "true to their inherent nature" (Schröpfer, Carpenter 2011, pg. 10). "A sensitive understanding of materials provides more than just a builder's means to a designer's end. Studies of materials inspire new understanding of power-to-whole relationships, organizational configurations, and phenomenological effects" (Schröpfer, Carpenter 2011, pg. 10).

Lighting has a series of functional roles beyond simply rendering our surroundings visible. It reveals the richness of materials - the rough texture of concrete, the translucence of etched glass, the gleam of polished granite. While materials possess physical properties, their effects can vary through use of designed lighting.

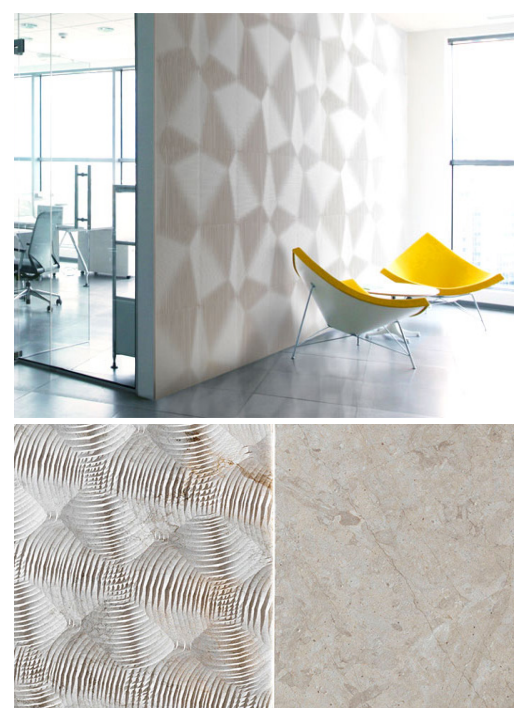

- F25

Textured Vs Flat Surface

Raffaello Galiotto - Lithos Design Domino Wall Panel light and shadow effects 


\section{Light Saturation}

The fascination of architecture with glass and its ability to admit light into interior spaces is evident throughout history. It was not until technological advancements allowed glass to be easily manufactured and made its use feasible that it became a common building material. Early glass design projects such as the Crystal Palace and Glass House introduced a new architectural design aesthetics that utilized modern technology and materials such as concrete, steel and glass. The saturation of light created an alternative spatial design that contrasted with traditional integration of lighting in designs. In the past, interior environments were commonly dark and gloomy with limited spatial illumination and outdoor connection. However, modern glazing created a seamless boundary that allowed light to flood into interiors. This experiential connection between interior and exterior environments became a popular design theme within contemporary architecture.

The invention of modern electrical lighting provided architecture with a reliable source of illumination, removing the dependency on sunlight for illumination of spaces. Artificial illumination allows for new spatial experiences and design expressions, redefining the way architecture is designed and experienced at night. Through the use of artificial illumination modern society 
has developed a night culture filled with social activities and events. The ability to control and manipulate artificial light offers a powerful design medium that is able to transform the characteristics and experience of architecture. Design application of artificial illumination considers the direction of light sources, as well as their intensity, brightness, hue and arrangement.

Glass can blur the boundary between interior and exterior environments, extending the perception of space between the two environments. When viewed from the exterior, the layers of perception can be described as follows: the foreground is considered to include the exterior façade and outdoor elements; mid-ground refers to occupants and furnishing, and the background consists of interior walls. People outside a building are able to view the activities within and vice versa. The resulting condition allows for multiple layers of objects, surfaces, materials, and light fixture to be compiled and composed into a single framed view that influences user perception.

Daylight is described as a phenomenon of the interior, artificial light is of the exterior. During the day, glass allows sunlight to be expressed and experienced within interior environments. It not only illuminates spaces but also creates light and shadow effects. The use of artificial illumination at

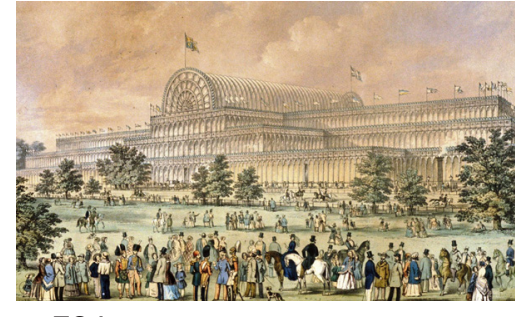

- F26

Crystal Palace

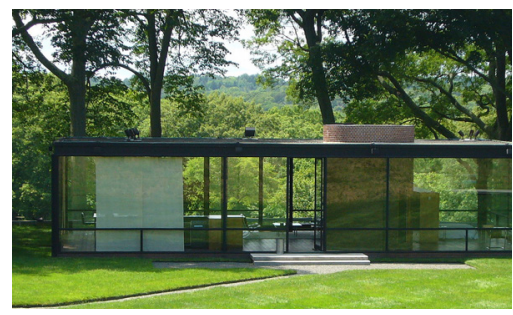

- F27

Glass House - Philip Johnson

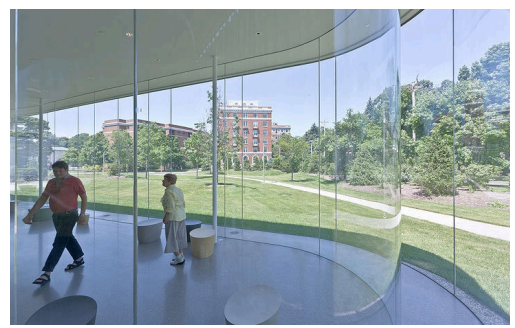

- F28

Glass Museum - Sanaa 


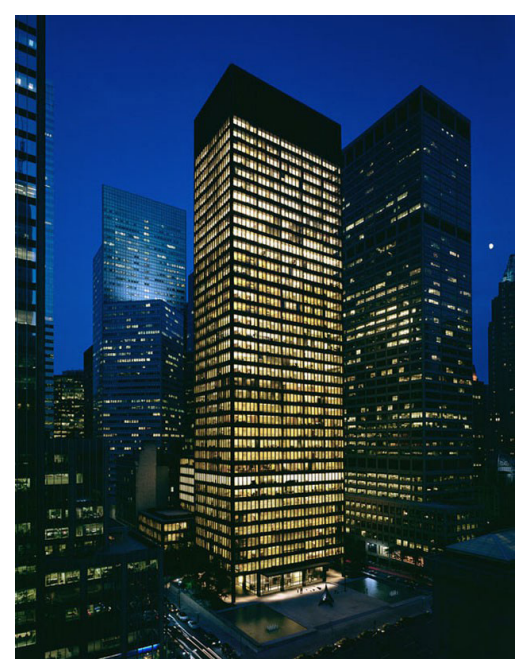

- F29

The Seagram Building - Mies Van de Rohe

Architecture as a lantern during the evening night enables the built environment to appear as illuminated objects on the street. James Carpenter, a light artist and designer believes that glass is one of the most intriguing material to work with, "The aim is to extend the experience of the boundary between the interior and exterior well beyond the physical depth of the building skin by understanding the optical and perceptual transition that occurs at that boundary" (Schröpfer, Carpenter 2011). Inherent within the physical properties of glass, is its ability to transmit, reflect, refract and absorb light based on the treatment of the surface.

Ease of construction, assembly and the unique effects of lighting has popularized the use of glass curtain walls in modern architecture. Regarded as one of the pioneers of modern architecture, Mies van de Rohe was fascinated with the potential of glass in shaping the architectural environment and experience. His obsession with reflective surfaces and minimalist design ideal were expressed within his designs, where they created perceptually stimulating environments that transformed throughout the day. Designed by Mies, the Seagram Building introduced a new architectural design expression through its use of glass and artificial lighting. The use of a glass façade allowed the building to reflect and display its surroundings, transforming its appearance as the sky changes colour. During the evening, the 
building perceptually transform into an illuminated object that displays its interior towards the city. The Seagram Building reveals the unique potential of glass and artificial illumination to create architectural environments that are perceptually stimulating throughout the day.

Partially the work of lighting designer Richard Kelly, the Seagram Building utilizes interior finishings and surface treatments to influence the exterior illumination during the night. The orange hue emitted by the building results from the use of wood within the office. The intensity and hue of the light expressed vary based on the type of wood used, its treatment and colour. Designing the light source is not the only method of expressing and experiencing light. As light interacts with surrounding materials, reflected light carries information along with it.

Kelly's research on lighting technology help introduce artificial lighting as a creative design expression within modern architecture. His essay 'Lighting as an Integral Part of Architecture' (1952) describes the effects of various modern lighting technologies and design methods as they are integrated and expressed within architecture. Kelly analyzed the type of lighting effects produced by a variety of artificial lighting sources and decided that the six qualities that must be controlled are
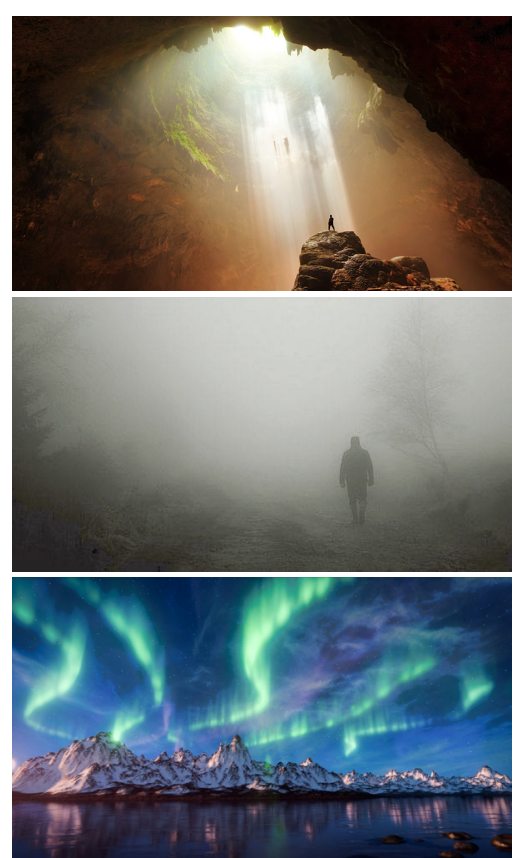

F30

Lighting Effects seen in the Natural Environment

1. Focal Glow

2. Ambient Luminescence

3. Play of Brilliance 
- intensity, brightness, diffusion, spectral colour, motion, and direction. This control means that the design of artificial illumination is not limited to codes and standards but has the potential to enrich architectural environment and experience.

Kelly further developed three lighting design principles in relation to the type of experience they offered within architecture. These concepts are (in contemporary terminology): 1. Focal Glow (Task Lighting), 2. Ambient Luminescence (General Lighting), and lastly 3. Play of Brilliance (Sparkle) (Kelly, Neumann 2010). Focal glow is described as being the focal spatial illumination within a space, a highly illuminated object that commands for attention. Ambient luminescence is the overall spatial illumination that is created through indirect or direct illumination. The last principle is described as a lighting spectacle, where the expression of light evokes a sense of imagination and wonder. The design of architectural environments that includes both a focal glow and ambient luminescence ensures an expression of lighting diversity that provides different forms of lighting stimuli within a space. 
42 | Light and Shadow Expression 


\section{Chapter 4 - Porosity}

\section{Contemporary Paradigm}

The expression of light in contemporary architecture has followed trends in modern lighting design techniques. The saturation of light through large glazed windows and artificial illumination define architectural lighting design within contemporary architecture. As concern for energy consumption continues to rise, the demand for energy-efficient and sustainable lighting design solutions has neglected qualitative light design integration.

"In modern times when it comes to designing with [natural and artificial light], designers, engineers and researchers refer to numerous codes and standards (e.g. CIBSE 1994), which elaborate primarily the quantitative recommendations but are lacking in offering overall qualitative advice. We know how to achieve a certain recommended illuminance level on, for example, the workplace of a sidelit office but we know little about how this office will appear to its user"

(Steane, Steemers 2004 pg. 180).

Marilyne Andersen describes the issue of architectural design within contemporary society, "In today's context of heightened environmental awareness, we feel pressure to evaluate architecture in terms of sustainable performance criteria" 
(Rockcastle, Andersen 2013, pg. 2). This tendency ultimately influences the integration of light within the built environment, as its artistic and poetic expression is often ignored in favour of practicality and efficiency. Michelle Addington, whose research explores energy systems, advanced materials, and innovative technology within the building envelope states that: "The search for an energy-efficient source drives much of the advanced research for many of the major lighting manufacturers" (Kelly, Neumann 2010, pg. 98).

Architectural design must 'perform' according to both qualitative and quantitative criteria, and we must work to re-establish the role of perceptual and preferential indicators in our language about performance. (Rockcastle, Andersen 2013, pg. 1). Research reveals that metric-driven lighting designs do not provide comfortable and visually engaging working environments, and so do not fulfil the potential of lighting. (Boubekri 2014). "We need to rebalance our definition of "performance" to include those perceptual and aesthetic aspects of light" (Rockcastle, Andersen 2013). William Lam's term "Lighting by Design, Not Engineering" references the desire to utilize light as a creative design medium in shaping architectural environments in relation to user perception and experience. While design arises from creative individual expression, engineering is based on functionality and efficiency. 
Quantifiable lighting design metrics ensure that interiors receive the required type of lighting for the performance of specific tasks. Whereas qualitative lighting design integration involves a creative design expression that influence the spatial ambiance and experience. Le Corbusier described this term as "eyes that do not see". It is necessary to develop standards in order to ensure all buildings receive correct design applications. Once a standard is met, future designs will be able to refine ideas and concepts based on preferences and design approaches. An example of this is the amount of light needed to provide adequate illumination on a desk. If the minimal requirement of lighting of a desk is 500 Lux (quantifiable metrics), a design possibility is to distribute the 500 Lux through a series of fixtures and interior finishings, rather than by means of a single output from the light source (perceptual / experiential design application). Light's design possibilities can be developed based on desired spatial ambiance and experience.

The effects of light on the human body, perception and experience is not yet fully understood, its investigation and integration within architecture is able to redefine the expression and integration of light (Boubekri 2014). A large body of research has examined how light expression within office environments can improve job performance and 


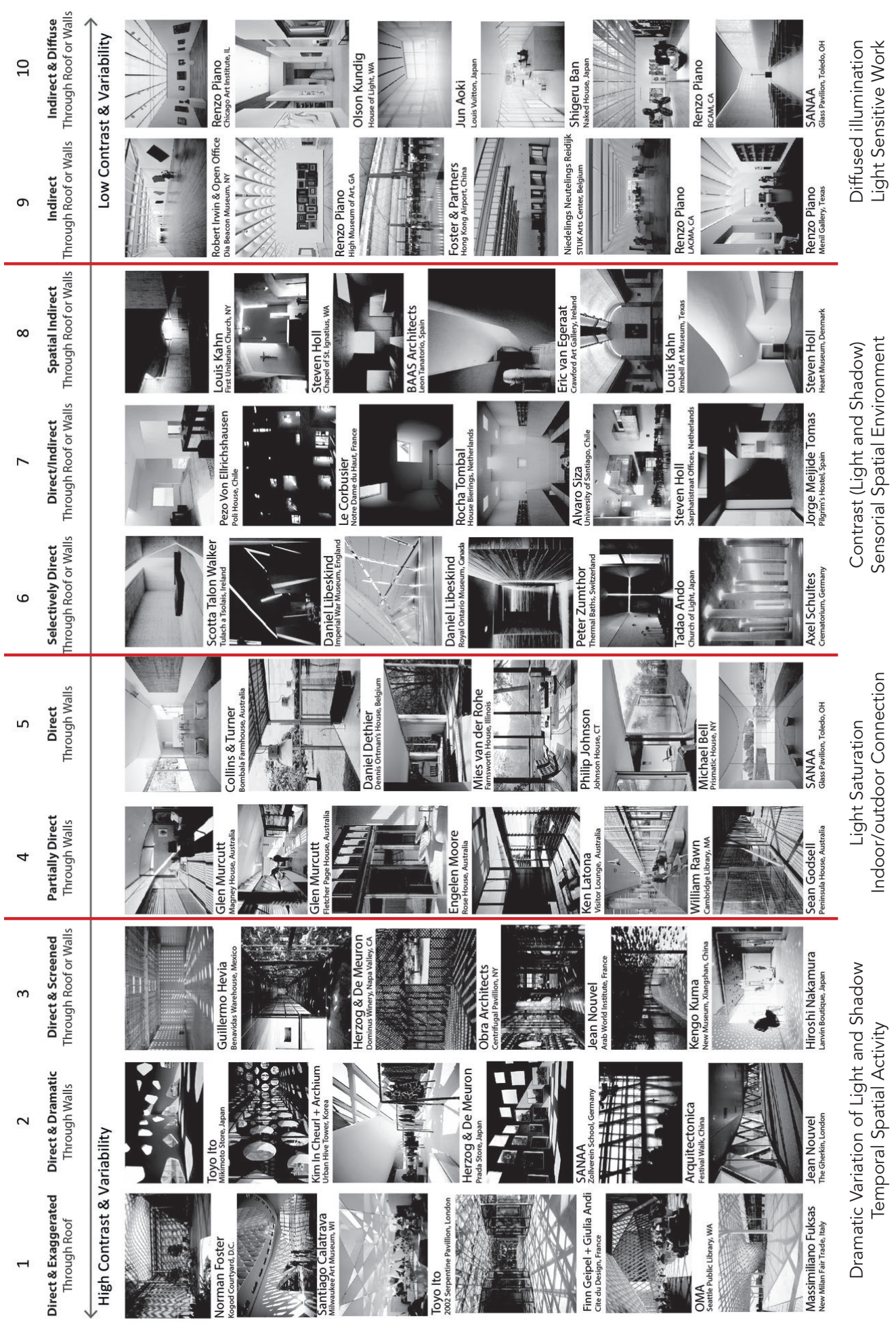

46 | Light and Shadow Expression 


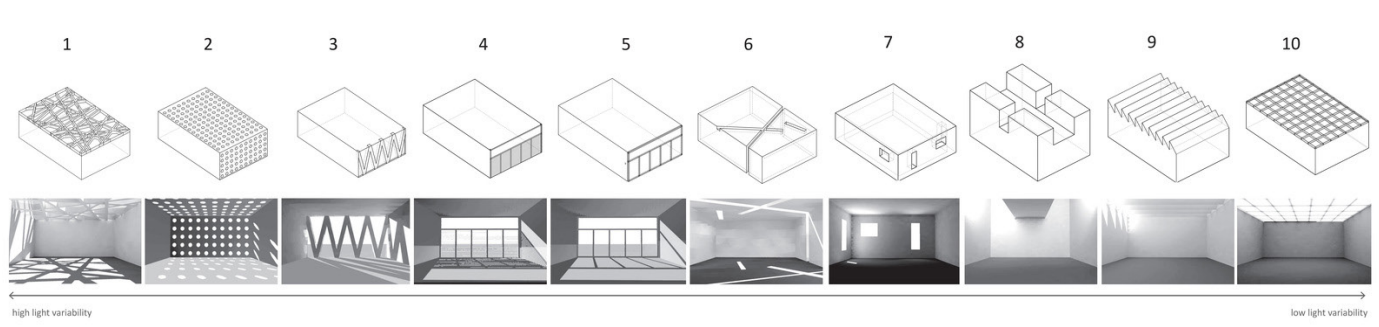

F32

Lighting Typology - Marilyne Andersen

Categorization of the types spatial lighting effects

- F31

Architectural Lighting Design Marilyne Andersen

Built works of different spatial lighting effects and experience within architecture. worker satisfaction (Boubekri 2014), Whether in an office, museum, warehouse, school, and etc... uniform distribution of artificial light floods the interior environment, maximizing function and efficiency (Kelly, Neumann 2010). Homogeneous lighting has deprived users of the potential experience of creative lighting design expression (Kelly, Neumann 2010). Our daily lives take place mostly indoors in spaces with uniform illumination. The outdoor environment is filled with lighting contrasts through an arrangement of different forms, materials, colours, and shapes, and a variation in lighting intensity.

The categorization of lighting effects within built architectural projects provides an understanding of the type of experience produced through particular spatial lighting condition. The resulting study revealed that the expression of light and shadow varies according to temporal conditions, providing visual stimulation within a space. Light is often used in circulation spaces, public spaces and open environments as they require open illumination. Low spatial ambiance and highlighting contrasts are designed for contemplative and religious buildings. When light is the focal point of a space, the effect can be spiritual and meditative. High spatial illumination is often expressed in residential buildings to provide indoor and outdoor connectivity. Lastly, the use 
of diffused illumination is integrated in lightsensitive programmatic activity, where consistent illumination throughout the day is required. There are definite correlations between programmatic uses and types of lighting (Rockcastle, Andersen 2013). The various lighting effects and design expressions showcase the vast potential for light to shape architectural environments and experience.

Helmut Bien discuss the role of lighting in shaping architectural spaces, in that "no more but also no less light: it should be appropriate... never treat all spatial situation in the same way, every space is different" (Bien, Helle 2010, pg. 7). The term 'appropriate' is in correlation to programmatic needs, user experiences and the type of ambiance designed by the architect. The expression of light within the built environment varies from dark contemplative space, bright public and social environments, calming diffused spatial illumination, and highly visual stimulating environments, and can involve a dramatic interplay of light and shadow. The characteristics and quality of light changes the experience and activity within a space.

The architectural experience can be described in terms of how light is expressed at any given moment in time (Plummer 2009). The integration of light offers architectural environments a poetic design expression as described by Norman Foster:

"If the spaces that we create do not move the heart and mind then they are surely only addressing one part of their function. Any engineer can quantify light by which 

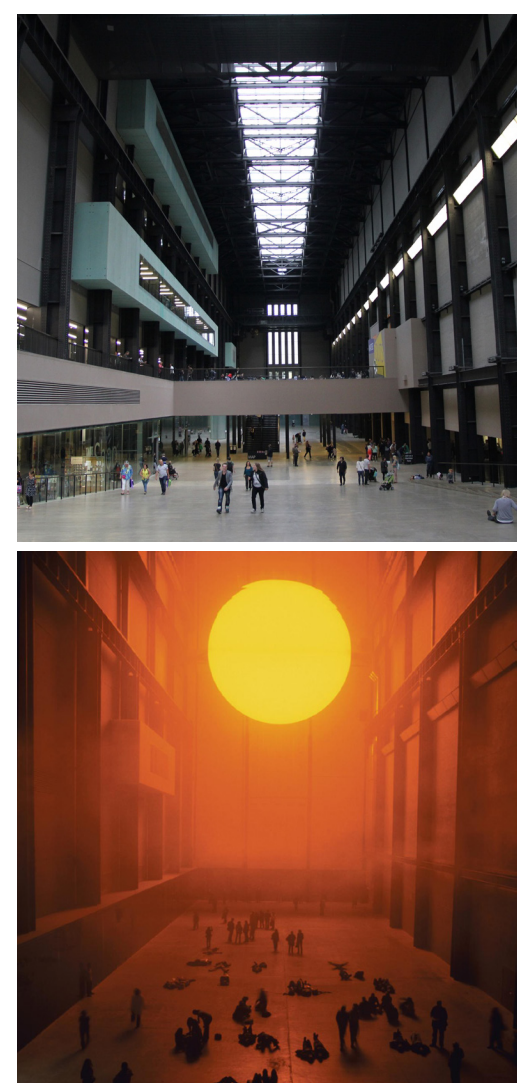

- F33

A - Tate Modern Museum -

Herzog de Meuron

B - The Weather Project - Olsfar

Eliasson

Poetic use of artificial

illumination to perceptual

transform the architectural

environment. to read a book. But what about the poetic dimension of natural light: the changing nature of an overcast sky, the discovery of shade, the luminosity of a patch of sunlight?"

(Entwistle 2012, pg. 6)

"[The integration of light within] architecture holds the power to inspire and transform our day-to-day existence. The everyday act of pressing a door handle and walking into a light-washed room can become profound when experienced through sensitized consciousness. To see, to feel these physicality is to become the subject of the senses" (Holl 2007, pg. 40). Norberg-Schulz describes architecture as a form of poetic and qualitative realities, "The primary task of the art of building, he declares, is to create a 'spirit of place' whose totality consists not only of concrete things with material presence, but also intangible things primarily light and atmosphere - which can inspirit buildings as well as landscape" (Plummer 2009, pg. 13). 


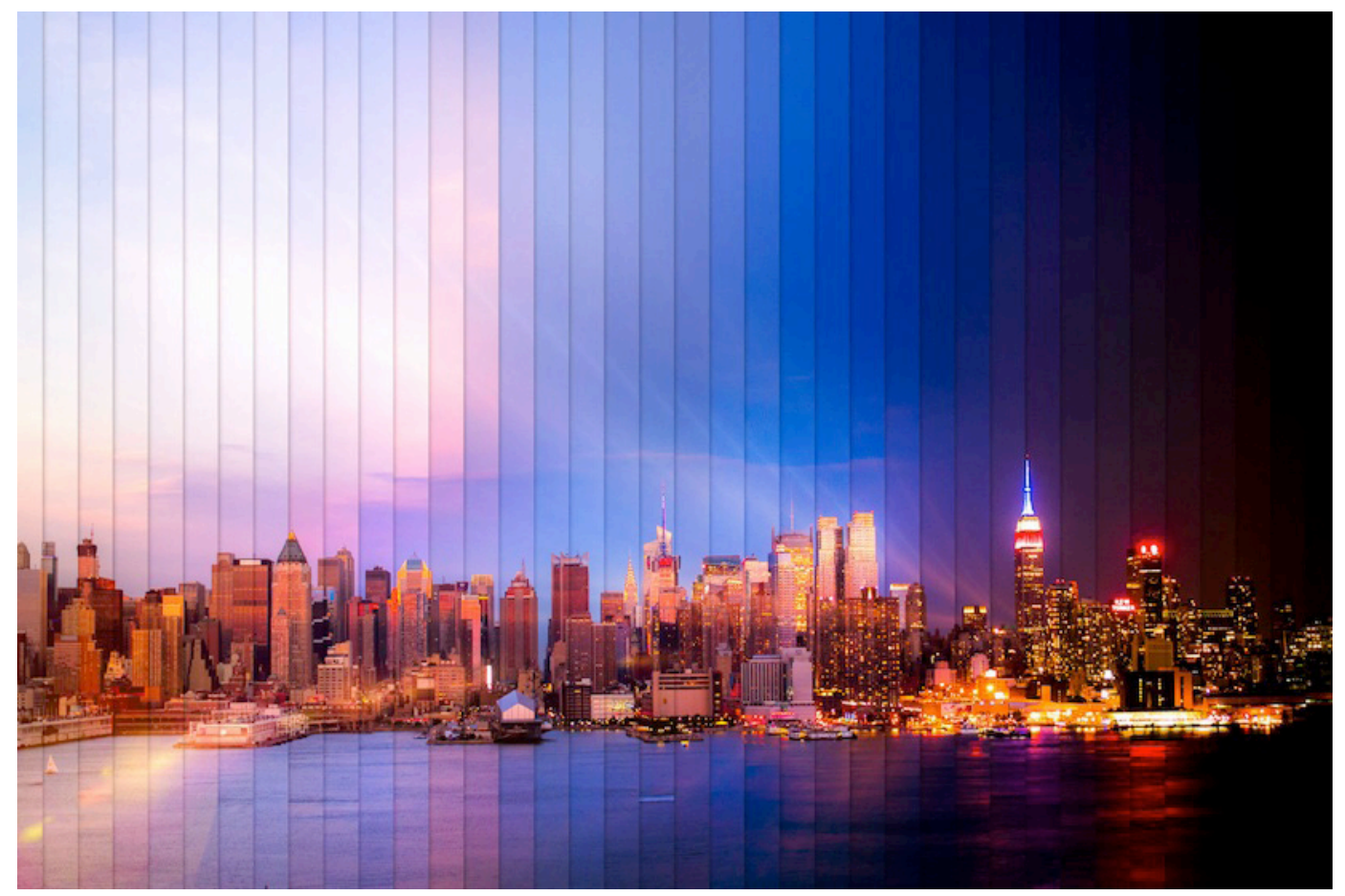

- F34

Day to Night Transformation in a City The perceptual transformation of the built environment throughout the day and night 


\section{Experiencing the Ephemeral}

The Earth, as it rotates on its axis, create varying degrees of sunlight exposure. The amount of light the environment receives varies throughout the day, and alters our perception and experience of the surrounding environment. Furthermore, the Earth's orbit causes seasonal changes and its different positions result in varying amounts of solar exposure for buildings. This dynamic transformation of light throughout the day and night provides endless perceptual stimulation. The ephemeral characteristics of daylight vary in such qualitative aspects as brightness, intensity, colour, orientation, and direction.

"Pallasmaa reminds us that light plays a crucial role in helping us become fully conscious of our existence in the world, and provokes interaction at an immediate, visceral and pre-cognitive level with the light we encounter" (Plummer 2009, pg. 13). The art of designing with daylight imposes creative discipline within architecture as traditional methods of spatial illumination are being challenged. (Steane 2011). The integration of daylight into structures requires an understanding of their environment, microclimate, shape, orientation and fenestration. It also necessitates some understanding of solar changes on the part of the designer (Frampton 2002). 


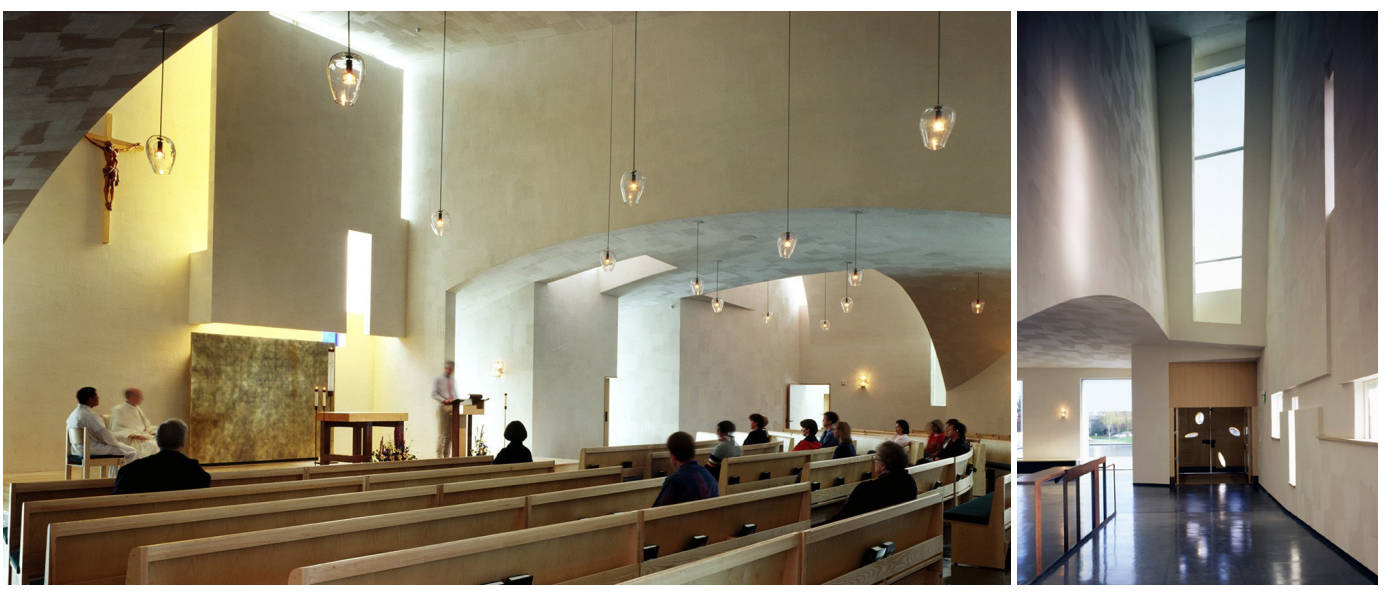

The presence of sunlight within the built environment is able to liven up the space during the day while silencing it at night. There is a continuous perceptual transformation over 24 hours. (Verges 2008). "The perpetual movement of light brings a dynamism within the built form that provides a relationship between people and their daily cycles" (Ramos 2015, pg. 47). Steven Holl states "the presence of [natural] light is the most fundamental connection force of the universe... [further described as the] twin entity which springs from matter and light" (McCarter 2015, pg. 123). The temporality of light can create provides structures with specific and unique conditions at different moments during the day. This experience surpasses any design ornamentation or architectural form as it can perceptually transform even as it engages multiple sensory receptors.

In "Capturing the Ephemeral" by James Carpenter, he states that the expression of light within architecture provides a temporal and sublime experience that transforms spatial environments at specific moments in time. The architectural environment appears to liven up under direct light exposure, and can seem to diminish or even disappear as lighting conditions change over time. Carpenter recognizes that light is a photon that contains information, travels through

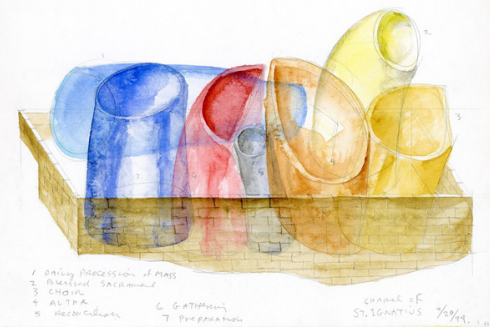

- F35

Chapel of St. Ignatius - Steven Holl

Colour and light as a design medium to enrich the architectural environment 


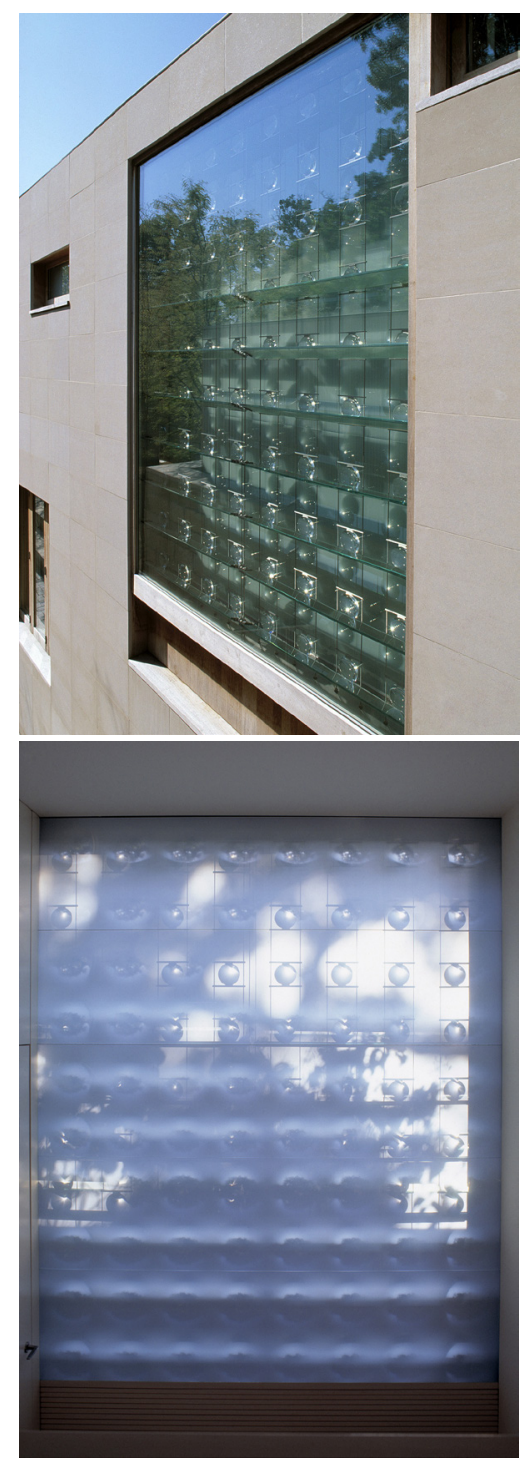

- F36

Periscope Window - James

Carpenter

A window design that captures light and colour of the surrounding environment space and reveals itself as it reflects off material surfaces (Schröpfer, Carpenter 2011). Light is often understood as only coming from energy sources, but in reality it is constantly being reflected and refracted from surrounding materials. "Each photon is a record of its journey and interaction, and we aim to understand and reveal this cumulative body of information" (Schröpfer, Carpenter 2011, pg. 108). The intensity and hue of the perceived reflected light varies based on the amount of light absorbed by the material.

James Carpenter expresses this understanding of light's interaction with materials in the design project Periscope Window. Here, a window feature is designed to allow natural light to enter and illuminate the walkway; at the same time, it distorts spatial perception by the use of reflected and refracted light. Each individual module contains a metal surface angled at various positions; it produces different lighting effects as it captures a variety of conditions through indirect illumination. An interplay of light and shadow is perceived in the wall assembly as individual materials capture different aspects of light. A compilation of multiple lighting expressions is superimposed into one experience as the window perceptually transforms according to solar orientation; at different times of the day, different hues are captured. Lights is expressed as "not to beautify, but rather 'to make 
men feel the rhythm marked by nature, harmonizing spaces with light, mitigating them with the passing of the sun (Plummer 2009, pg. 11).

The use of volumes, forms and materials can add experiential depth to light's temporal characteristics as the architectural environment changes throughout the day. Light is not bound to the same restrictions as is material; its dynamic nature means it is constantly changing our perception of space. Steven Holl's understanding of the importance of light to design in terms of perception and experience has resulted in an expression of light phenomena in his projects. His design philosophy integrates light into the built environment as a creative artistic expression, one that animates space and alters the user's experience throughout the day (Mosco, Holl 2009).

"[Natural] light is never static, always on the move, constantly and elusively shifting throughout the hour, day and year. These changing qualities of natural light define both visual appearance and atmosphere over time. It is perhaps this less tangible, more ephemeral side to light that creates memorable and enjoyable places and experiences"

(Major, Speirs 2005, pg. 16).

In the design of the Church of St. Ignatius, Holl utilized light and color to animate the interior. It was based on solar orientation: sky-lights, lightwells and windows introduce different hues into the space. The amount of colour that is perceived 


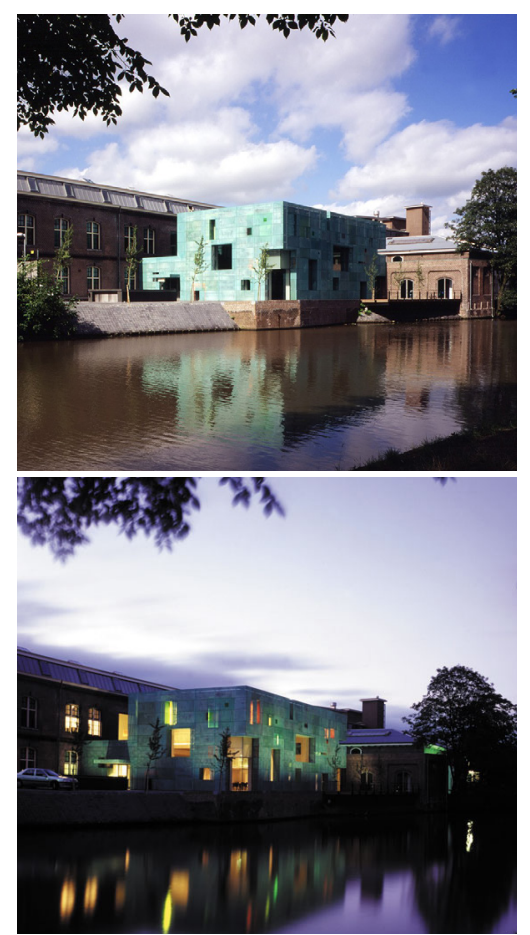

- F37

Sarphatistraat Office - Steven Holl

The use of artificial illumination to perceptually transforming the built environment is a result of light intensity: the greater the intensity of the light source (the sun), the more reflected light there is and colour is therefore washed onto the interior walls. The expression of light within the built environment is able to transform something inert and dead into spaces filled with energy and spirit. Light is the most spectacular experience that engages perception and enhances the experience of architecture. The church panels are also integrated with artificial illumination, producing effects similar to that of sunlight within the space. The effect is a distortion of the user's sense of time, as the light source is hidden from him or her.

Through the use of artificial lighting, the built environment is able to redefine the way users experience and perceive it during the night. Sunlight is the primary source of illumination during the day while at night artificial illumination takes control. Artificial illumination allows modern cities to be operable for 24 hours a day, creating cities that never sleep. Holl poetically describes this perceptual transformation of light within the built environment: "In the mist of the metropolis at night, space dissolves before our eyes, only to take shape again within seconds" (Holl 2000, pg. 17). Buildings can appear as glass forms during the day, and transform at night into luminous objects. "An individual work of architecture in its city space may have an entirely different presence by day and by night, both equally important as the self-contained "object" claims a large, less contained presence (Holl 2007, pg. 69). The creative expression of artificial illumination offers architecture possibilities for new sensory effects and experiences. 


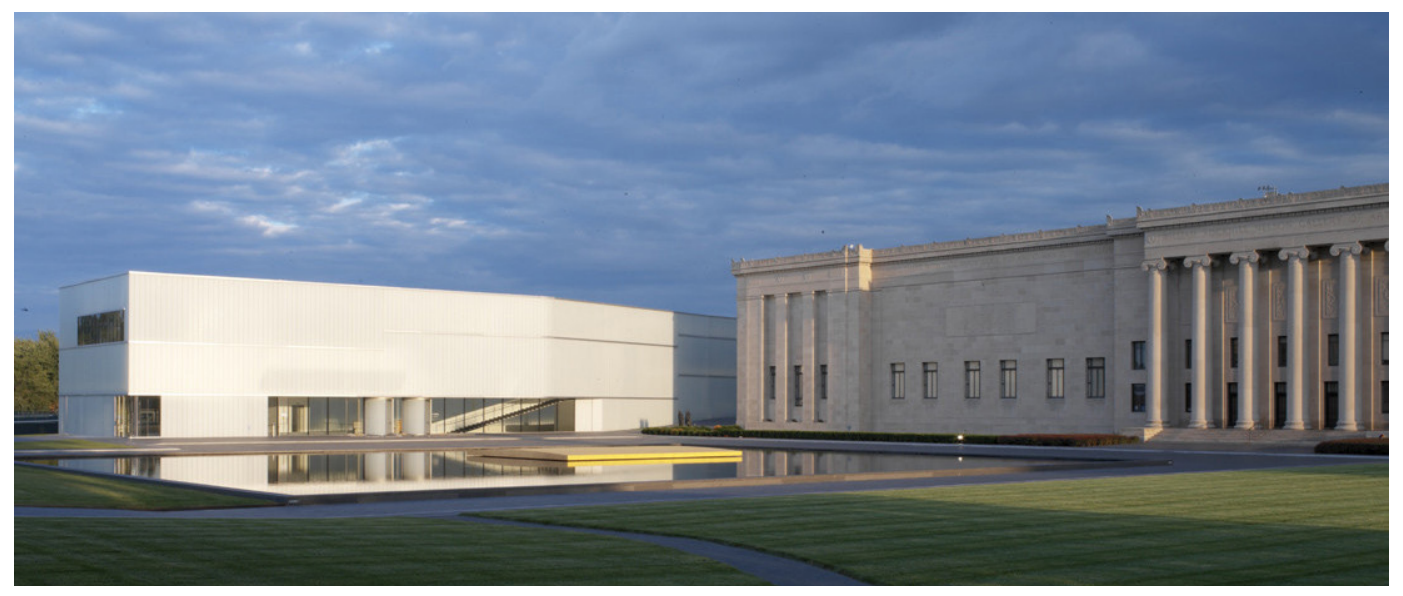

Architectural integration of "artificial lighting was usually viewed as a 'system' rather than a 'medium', and it was not until the last two decades of the twentieth century that architects eventually began to embrace artificial light as an integrated part of the design process, rather than appended as a functional system, or indeed, a cosmetic addition" (Major, Speirs 2005, pg. 17). Technological constraints limited the creative design expression of artificial light, where the invention new lighting technology in the 21st century has allowed for more design control and opportunities as well as efficient use of energy, allowing the expression of artificial to create experiential environments that surpasses the sun (Major, Speirs 2005)

The concept of artificial light as a design medium is expressed in the design of Atkins Museum by Steven Holl. The use of C-channel glass as the façade material allows light to enter the building during the day, as well as to appear flat and less dominant within its surroundings. The design attempts to allow the historic building near the museum to become the focal point for tourists and visitors during the day. At night, it is transformed into a luminous object through the use of artificial illumination. Its closure during the evening allows its contemporary design to become its focal point. This duality of lighting expression is seen in the

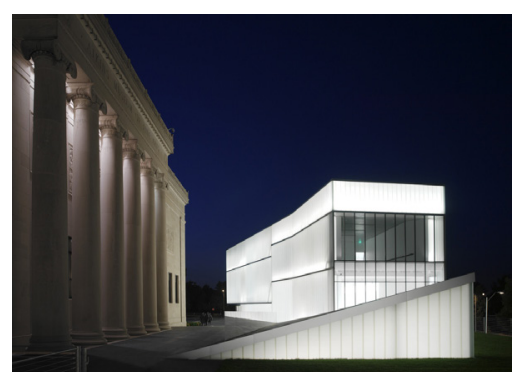

F38

Atkins Museum - Steven Holl

Day and night comparison of architectural lighting 
contrast between solid/dark traditional architecture and the porous/light contemporary design.

The increased use of artificial illumination within the built environment has made it an important element of design that can be expressed and integrated within structures. Artificial lighting, like sunlight, has a unique role in shaping the architectural experience. "Artificial sources offer as many possibilities for expression and the creation of architecture as the light of the sun itself" (Major, Speirs 2005, pg. 3). Artificial lighting has become very important in the development of modern-day architecture, Louis Khan expresses the danger of relying solely on artificial illumination in architecture. "It should not supplement daylight, as no space, architecturally, is a space unless it has natural light" (Major, Speirs 2005, pg. 15). The expression of both daylight and artificial illumination has innovative architectural potential. 
58 | Light and Shadow Expression 


\section{Chapter 5 - Light and Architecture Architecture as a Vehicle to Express Light and Shadow}

Light is a powerful design tool that can transform mundane spatial environments into perceptually fluid and stimulating ones through its articulation and interplay with shadow. This thesis considers an office facility as a vehicle for the creative articulation and expression of light and shadow in order to generate a positive and distinctive architectural experience and spatial environment. This office caters to small start-up companies, providing users with collaborative and temporal working environments that respond to the 24-hour lifestyle of contemporary society. The integration of light, and its changing conditions, within the office hub supports its programmatic needs even as it provides an enriching spatial experience as the lighting condition changes throughout the day. The design of lighting concepts provides workers with a range of lighting conditions and spatial environments that are responsive to the user's working habits.

This design research project explores light as a creative and poetic design expression. It critiques the issues of homogeneous spatial lighting environment and light saturation within contemporary architecture. The pervasive use of glass curtain wall and lack of architectural design integration of artificial illumination has deprived users of the enjoyable experience provided by the interplay of light and shadow. The characteristics and qualities of light when it is integrated into spatial environments can transform the way users experience and perceive architecture. The articulation and expression of both sunlight and artificial light within office hubs offers new experiential opportunities for day-time and nighttime. 


\section{Lighting Design Strategies}

Utilizing light as the primary design driver for an architectural experience within spatial environments, this thesis design project explores the impact of creative lighting design integration on user experience and perception. The design strategies developed are methods to curate and express light within the built environment. The articulation of sunlight, artificial light, architectural form, physical properties of material, and spatial ambiance offers the potential to create perceptual, fluid and stimulating environments that can transform the way users experience and perceive the built environment over the course of the day and night. 

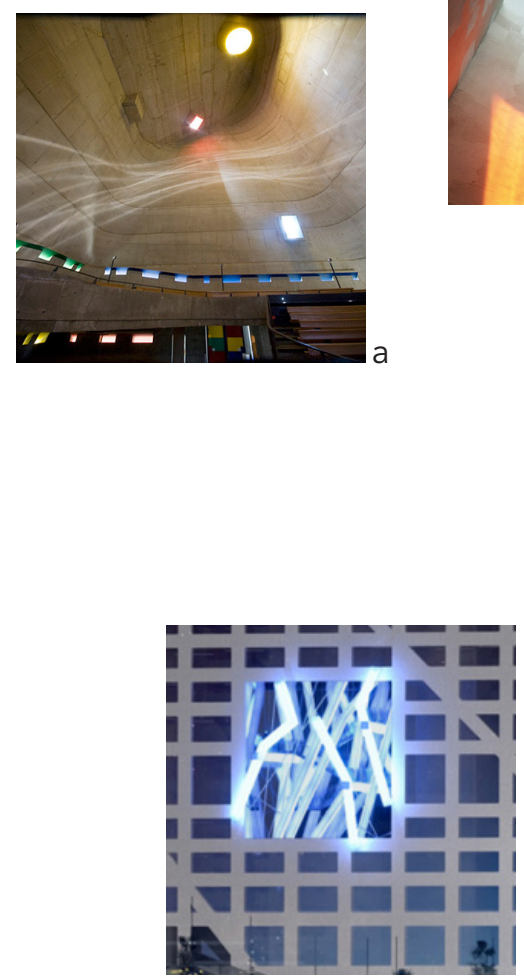

a

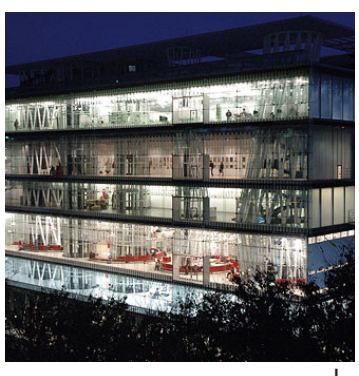

b

- F40

Artificial Light Expression

a: Lebbeus Woods - The Light

Pavilion

b: Toyo Ito - Sendai

Mediatheque

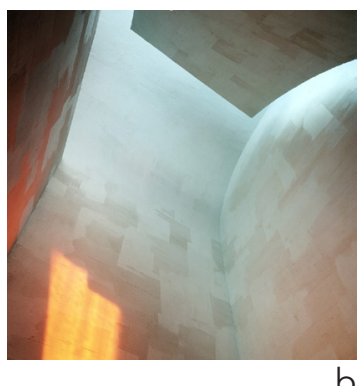

- F39

Sunlight Integration

a: Le Corbusier - Church of Saint-Pierre

b: Steven Holl - Chapel of St. Ignatius

Strategy 1: Solar Orientation as Architecture Experience

The first strategy utilizes solar orientation as a means of designing, shaping, organizing and generating an architectural experience within the spatial environment. The architectural integration of daylight correlates to the user's specifications regarding experience, ambiance and programmatic need. The power of the spatial environment is such that it can be designed for high solar exposure, producing energetic and engaging environments, or low exposure to provide intimate and calming environments. The effect of sunlight as the sun moves across the sky provides the opportunity for the built environment to creatively integrate and reveal light to its user, creating spatial environments that are experience at specific moment in time.

Strategy 2: Artificial Illumination to Perceptually Transform Architecture During the Night

The second strategy explores artificial light as a creative design expression, one that will redefine the way users experience and perceive built environment during the night. The ability to control and illuminate space on demand presents different challenges from dealing with natural light, sunlight, 

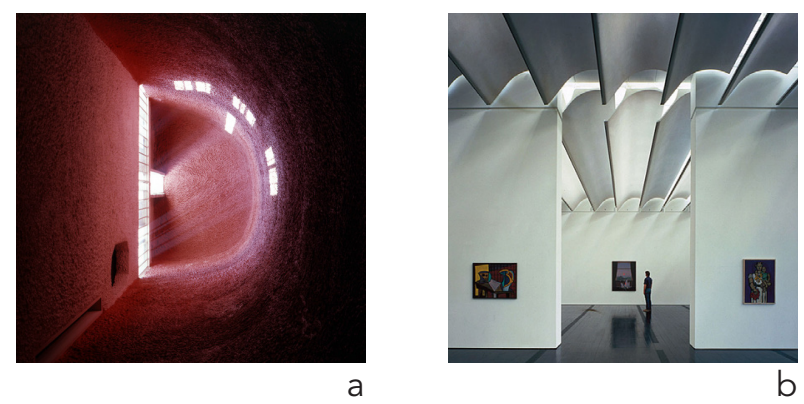

- F41

Architectural Form and Light

a: Le Corbusier - Ronchamp

b: Renzo Piano - Menil Collection

although there are similarities in the use of both. A milieu that appears dull and lifeless during the day can become dynamic and interesting under the night sky. The ability to control and manipulate artificial light offers a powerful design tool in when shaping specific environments and creating an overall architectural experience.

Strategy 3: Architectural Form to Express and Reveal Light

The third strategy utilizes form as a means to articulate, integrate and reveal light within architecture. Forms provide the opportunity to creatively express light within the built environment; they have the potential to integrate light in cases where there are constraints on natural light exposure due to existing site conditions. Designing the architectural form with emphasis on its relationship to light can produce powerful effects of light and darkness.

Strategy 4: Material Properties to Reveal the Characteristics of light

The fourth strategy explores the physical properties of material as a method to articulate and express light within architecture. Materials can perceptually
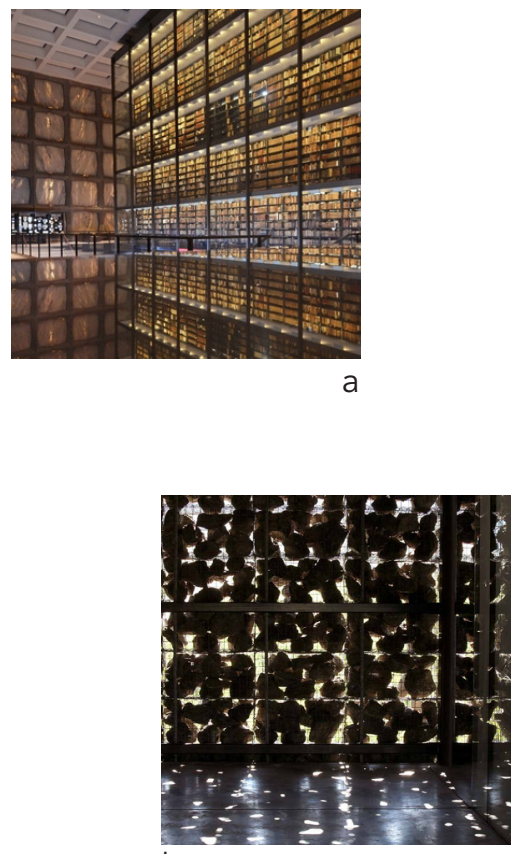

b

- F42

Material Properties and Light

a: Gordon Bunshaft - Rare

Book Library

b: Herzog \& de Meuron -

Dominus Winery 

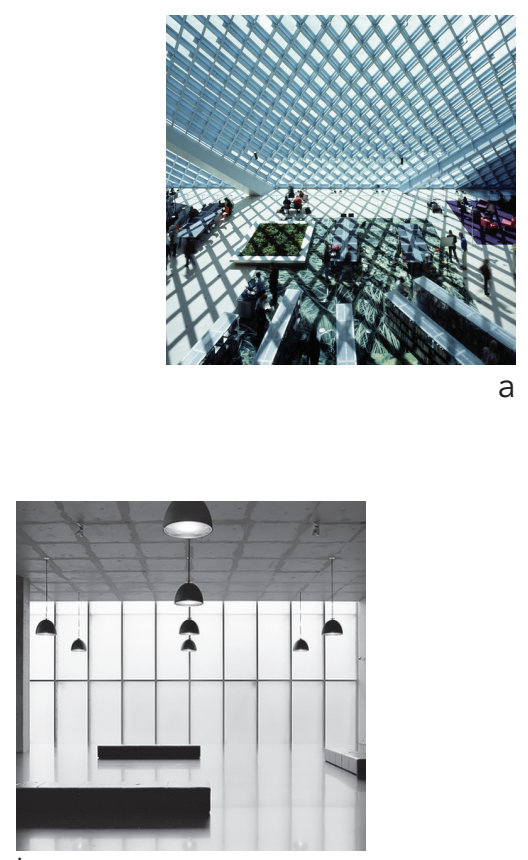

b

- F43

Spatial Ambiance

a: OMA - Seattle Public

Library

b: Peter Zumthor - Kunsthaus

Bregenz transform their locations, whether the lighting is low or high, whether the illumination is direct or indirect. The physical properties of material, its assembly and construction, in combination with its interaction with light, can transform the way users experience and perceive built environments.

Strategy 5: Spatial Ambiance to Enrich the Architectural Experience

The last strategy examines light in relation to the type of experience and spatial ambiance it produces within the built environment. The type of lighting a space receives describes the function and characteristics of a space. A bright spatial ambiance evokes a public and energetic environment, whereas low spatial illumination suggests privacy and contemplation. 


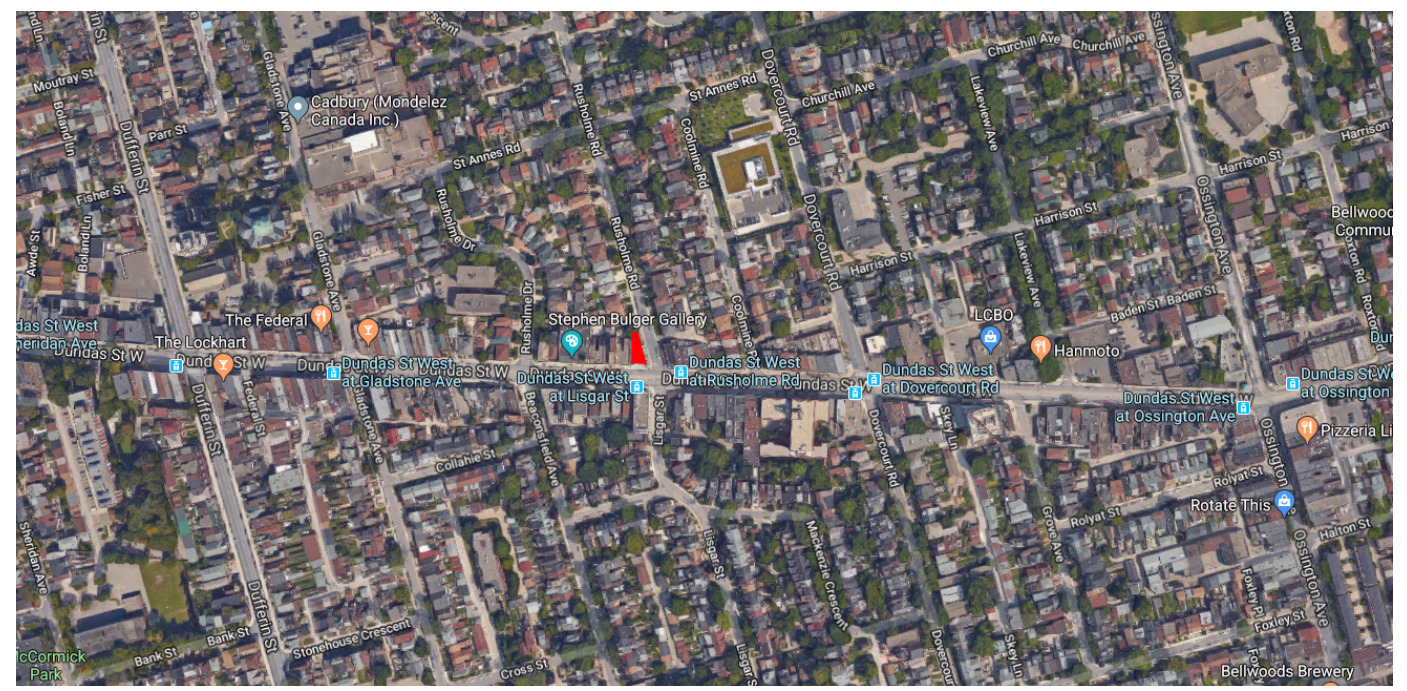

- F44

Site Location

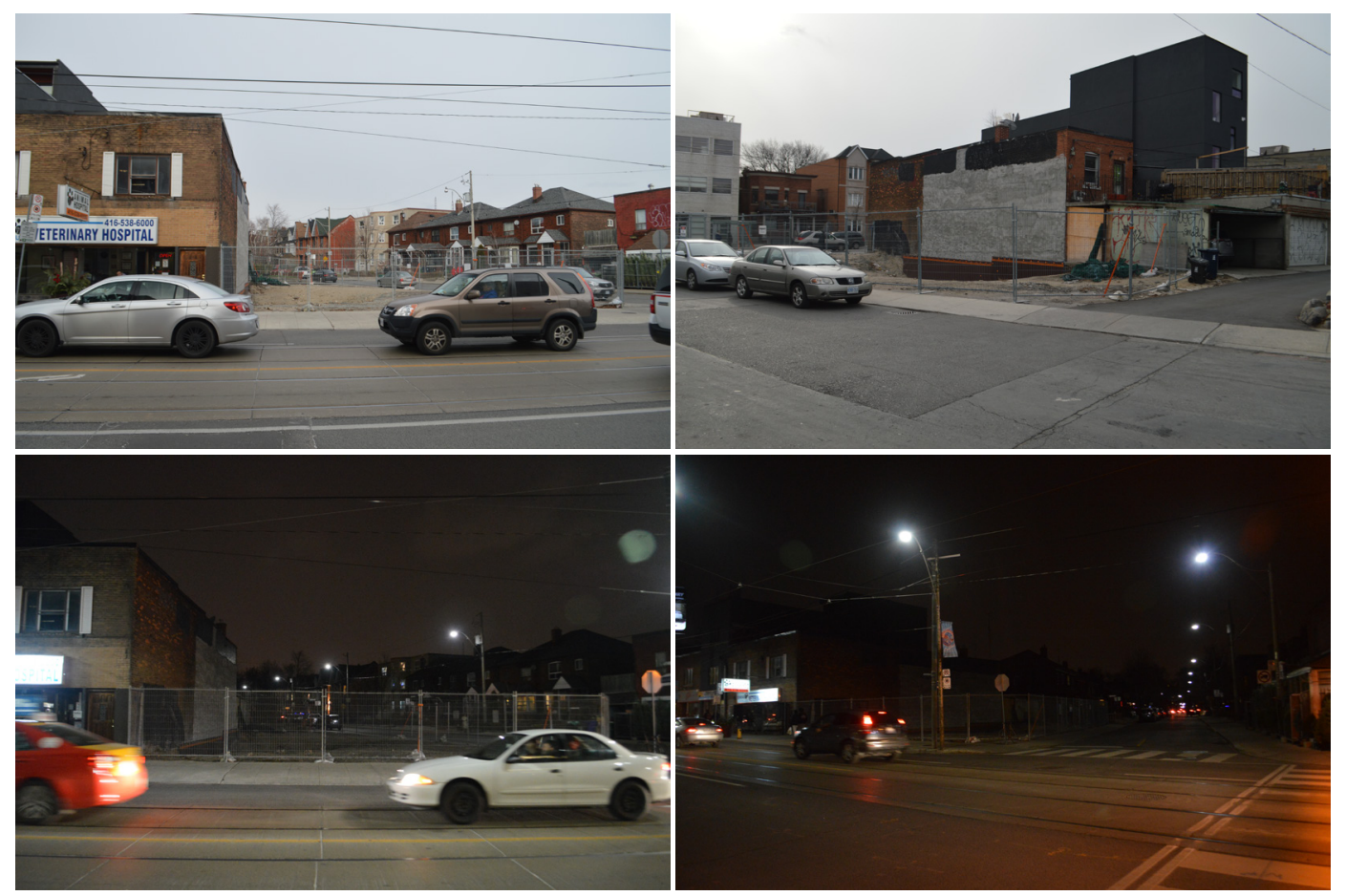

- F45

Site Photos

64 | Light and Shadow Expression 


\section{Site Description}

Daylight integration within architecture requires an analysis of the existing solar patterns of a site, which will provide designers with an understanding of design challenges and opportunities. Site conditions such as neighbouring buildings, geographical location, climate condition and street characteristics influence the expression and integration of light within a specific structure. Utilizing light as the primary driver for design, this thesis project utilizes both sunlight and artificial light for the generation of an architectural experience within a spatial environment.

The site of the thesis design project is located on 1347 Dundas Street West, Toronto Ontario, Canada. Toronto's geographical location results in seasonal changes throughout the year, changes that influence the amount of light buildings receive throughout the day. The city receives up to 10 hours of direct sunlight exposure during the winter and up to 18 hours in the summer. During the winter, lesser light exposure can cause seasonal affective disorder (SAD), which has a negative impact on humans' overall mood and physical aptitude. The built environment has the potential to counter such conditions, with the integration of light to support and influence user comfort and well-being.

As seasons change in the city, solar angles provide design challenges associated with the integration of light. The peak solar angle at noon ranges 
from $30^{\circ}$ in the winter to $70^{\circ}$ in the summer, providing an array of possible spatial lighting exposure and experiences throughout the year. The understanding and consideration of solar angles can aid the designer in the attempt 0 reveal and utilize light as a medium. In the colder seasons, between October and March, direct solar exposure is wanted within buildings as a method to passively heat and illuminate spaces. However, in the warmer season between April and September, it is necessary to reduce the amount of direct solar exposure to avoid excessive heat gain within interior environments.

The site is a corner lot consisting of street frontage along the south and east façades. The design has the potential to integrate and articulate different lighting methods along the individual facades. The programmatic functions and typologies of buildings along the adjacent streets differ from one another; the south-facing street primarily consists of commercial buildings, while the east-facing street accommodates residential housing.

Dundas Street West to the south of the site is a highly energetic street with pedestrians and cars passing by throughout the day and evening. Small commercial buildings that populate the street serve surrounding neighbourhoods. They utilize large, glazed windows to create a public and inviting impression during the day and night.

Towards the north of the site is a residential neighbourhood consisting of detached and semidetached houses. The transition from an active, public atmosphere to the calm one of a private residential neighbourhood presents the opportunity to create different lighting scenarios along building façades. To the west of the site is a commercial building that provides design challenges as its west façade is limited as to the amount of available openings.

This thesis project will take advantage of different lighting conditions along the east and south 


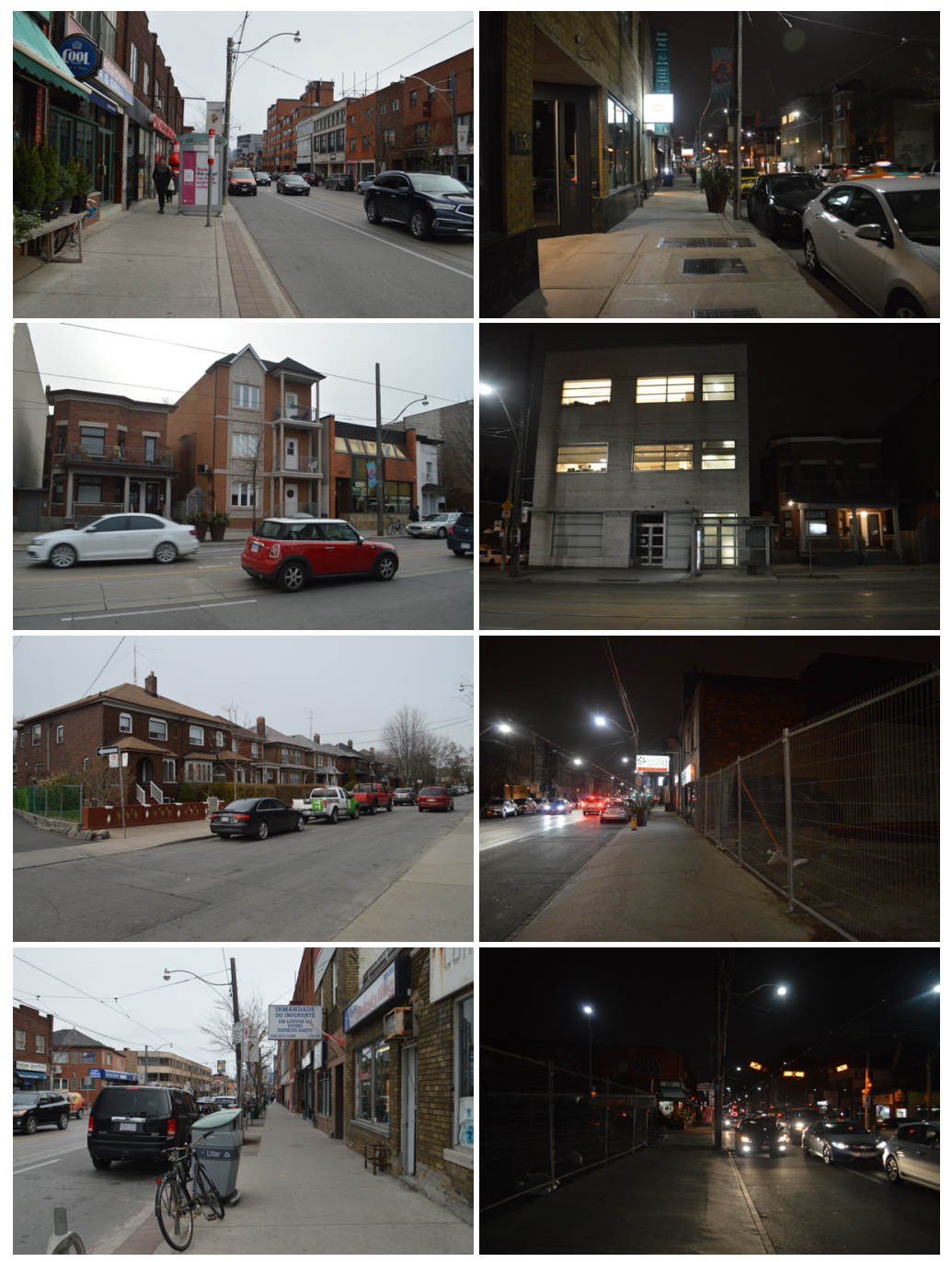

- F46

Neighbourhood Photos

Day/Night Characteristics

façades and will utilize roofs for the introduction of sunlight in areas with limited direct solar exposure. Consideration of the site's conditions and programmatic needs will influence the end result as regards spatial experience and perception. 


\section{Program Description}

The working culture of offices in the 21 st century has significantly evolved; this dynamism has influenced office design and structure, making it different from that developed in the early 20th. Contemporary offices are more mobile, collaborative, and interdisciplinary than their predecessors; the traditional office design typology does not support these cultural changes. Traditional office typology focused primarily on function and efficiency, utilizing large open-floor plans and grid layouts for lighting fixtures and furniture. The result was spaces that were homogeneous and mundane, lacking visual stimulation and depriving users of the experience that the interplay of light and shadow could provide.

The integration of light within an office has the potential to change the way users experience and perceive spatial environments across the 24hour working lifestyle of contemporary society. The innovative expression of different spatial lighting environments during the morning, afternoon, evening, and midnight, can provide built environments that support the working habits of users. Office workstations, collaboration rooms, meeting rooms and lounges must be designed to creatively express different spatial lighting conditions, altering the way users experience environments throughout the day, respond to them, and behave generally. 

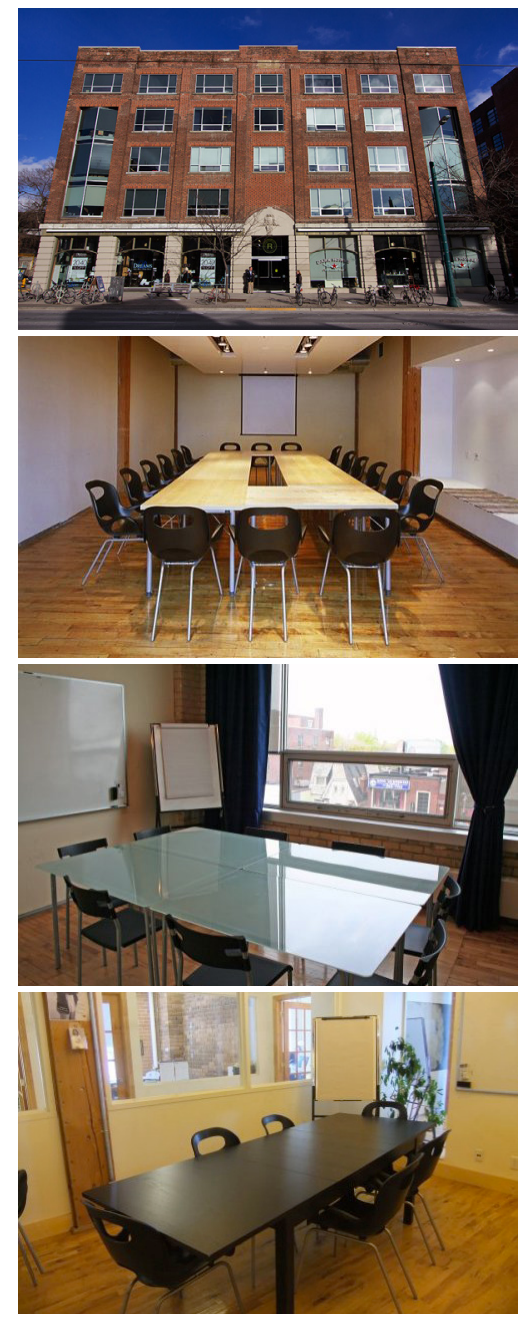

- F47

Centre for Social Innovation Exterior Facade / Interior Meeting Room
The success of the Centre for Social Innovation (CSI), and its growth within Toronto has demonstrated the demand for temporal/shareable office working environments, ones that cater to start-up companies. CSI is designed to house multiple small local business in a single building, attempting to provide temporal spatial working environments that will support social entrepreneurs and like-minded individuals. The office facility enables businesses to gather and support one another, and allows users to share ideas and experience, educate others, and develop strategies for future growth.

Theirgoal isto provideflexibleworking environments based on user demand and preferences throughout the day; however, their facility was not designed in such a way as to cater to these principles. Its spatial layout, uniform façade treatment and materials selection gives it a homogeneous expression that lacks an expression of individual identity. Such an expression would impact users' habits, response to their workplace, and behaviour generally. Using light as a design medium to create the architectural experience. The expression of light proposed in this thesis project will re-define traditional office working environments, and create unique spatial lighting conditions to engage with user activity and enhance the architectural experience.

The facility consists of temporary work stations, team clusters, meeting rooms and event spaces, as well as a lounge, classroom and kitchen that are open to all users. The provision of temporal work environments allows users to move freely around spaces according to their preferences and activities 

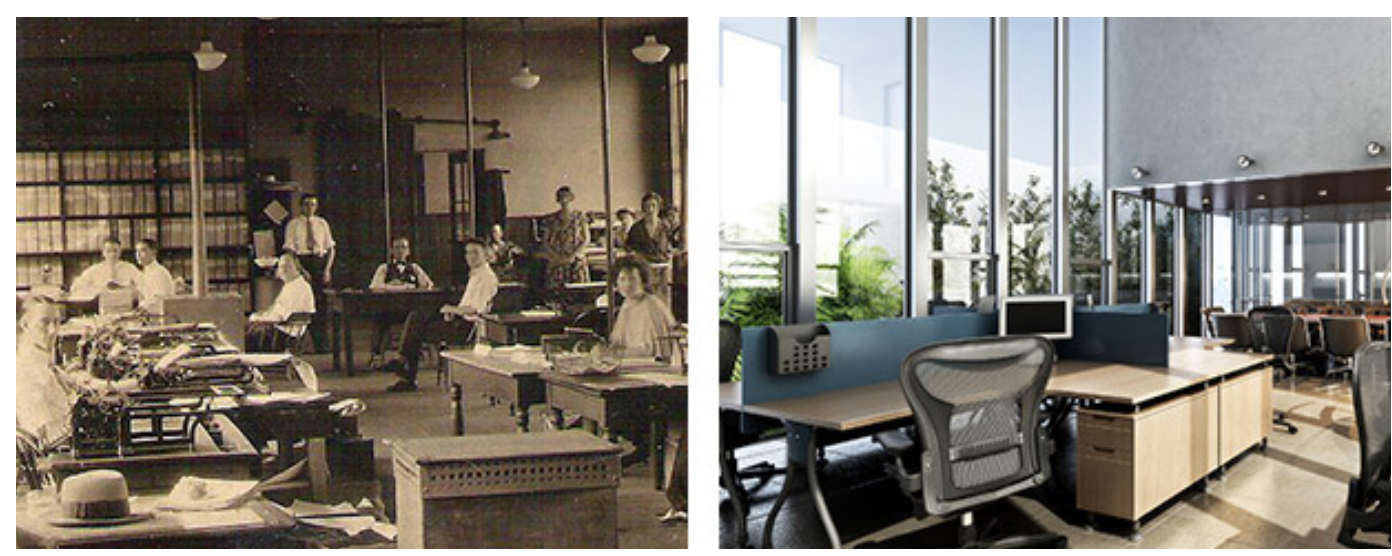

throughout the day. Users are able to work in spaces that receive direct light exposure during the morning, afternoon, and evening, and can enjoy environments that are collaborative and private. The office hub has the potential to express different lighting conditions to enhance user experience throughout the day. The organization and location of apertures and their design for filtering light can contribute to a working environment that has a unique identity and offers its users an innovative experience.

Programmatic lighting design concepts are:

1. Circulation space - low light sensitivity

a. Minor task illumination

b. Temporal use throughout the day

2. Multipurpose / collaborative space - Light variability based on activity

a. Specific to its functional use

b. Group / social activity

3. Work Space (Hot desk) - Direct solar exposure for selective rooms along the east, south, and west facade
a. High task illumination
b. High usage
c. Enjoyable spatial ambiance
d. Individual activity

4. Lounge/collaborative space - Dynamic lighting environment
a. Visual stimulation
b. High usage

- F48

The Office Environment

1920s / 2000s office

organization 
c. Group activity

5. Meeting spaces - Ranging for bright to dark

a. Presentation room (projection - dark)

b. Collaboration room (white board -

bright)

c. Meeting room (talking - bright)

6. Private group office - Enjoyable spatial

illumination

a. High task illumination

b. High usage

Within our daily lives, we experience a variety of different lighting scenarios that captivate our attention and stimulate our senses. Our field of view is constantly changing outdoors, providing visual stimulation through different lighting effects. The vivid display of colours, textures and design features provides us with scenic and pleasurable experiences. The natural environment is filled with a diverse range of memorable lighting effects and experiences; these include glimmering stars in the night sky, the single beam of light in a cave, the hot sun on a beach, the diffusion of light through clouds in the sky and the filtering of light through leaves on a tree.

The design of the thesis project utilizes different light scenario within each individual spaces, creating a distinct spatial ambiance and experience within the office hub. A poetic and artistic design expression of light is able to transform the way user perceive the built environment throughout the day and night. The characteristics, experience and quality of light within the natural environment provide lighting design concepts and objectives that creatively express and integrates light. The lighting experiences engendered by a cave, the sky, and forests provide unique sensorial and perceptual stimulation that are captured within the design of spaces of the thesis project. Taking lighting concepts and experiences of the natural environment provides an opportunity to reveal and experience light, changing the expression and integration of light. The lighting effects of are as followed: 
1. Cave - (Private, Meeting)
a. Highly rich in texture
b. Granular material
c. Low light ambiance
d. High contrast and selective opening

2. Sky - (Workstation, Energetic)
a. Layered material
b. Diffused illumination
c. Luminous environment
d. Continuous illumination

3. Forest - (Vertical displacement, field of depth)
a. Depth of field
b. Rhythmic patterns of light and shadow
c. Shifting lines
d. Vertical displacement

Utilizing the sky as an example of an overall spatial lighting design goal, the spatial environment can be designed to have a high lighting ambiance through indirect or diffused illumination. A highlighting condition makes users feel more awake and alert for social activity and is conducive to a more productive working environment. This condition contrasts with the spatial environment of a cave. The low-light ambiance creates a sensorial environment that deprives users of visual stimulus, creating a private and intimate spatial environment. The poetic expression and experience of light can support the practical functioning of an office even as it enriches the architectural experience over the course of the day.

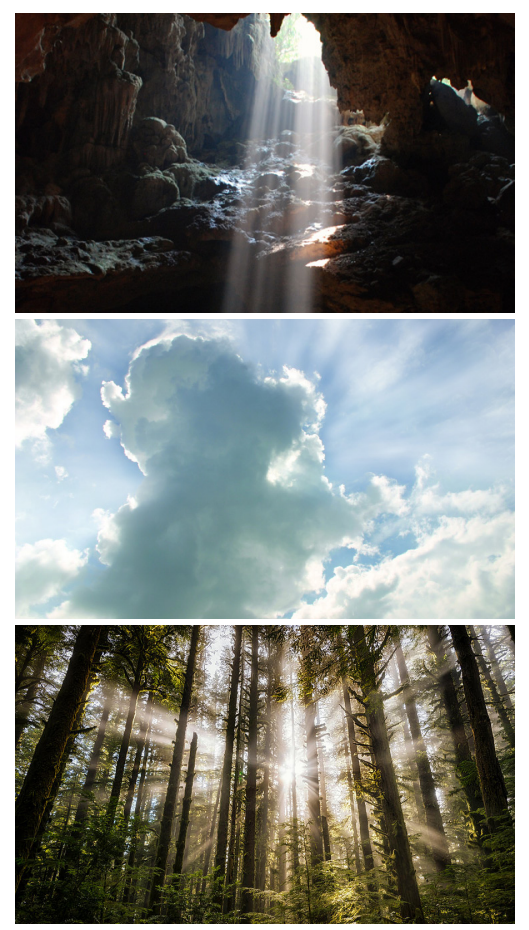

- F49

Lighting Effects Design

Concepts

a: Cave

b: Sky

c: Forest 


\section{Design Project}

The following drawings showcases the thesis design project through a series of architectural drawings, renders and diagrams. A sequence of images are presented, showcasing the type of lighting design effects and expression within the office hub. 


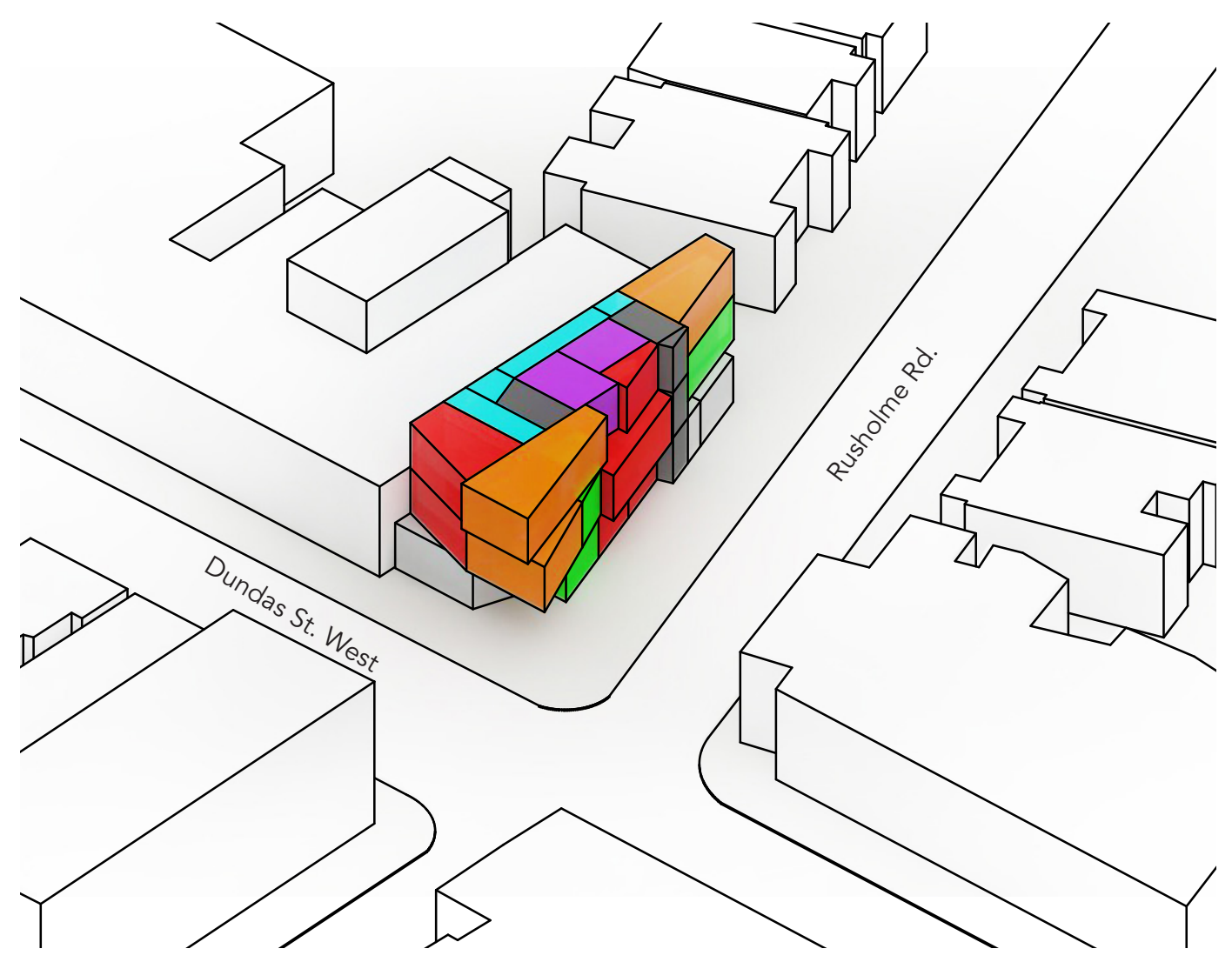

- F50

a_Orthographic Drawings

Programmatic Organization

Hot Desk

Collaborative Space Meeting Rooms

Support Space

Private Offices

Circulation 


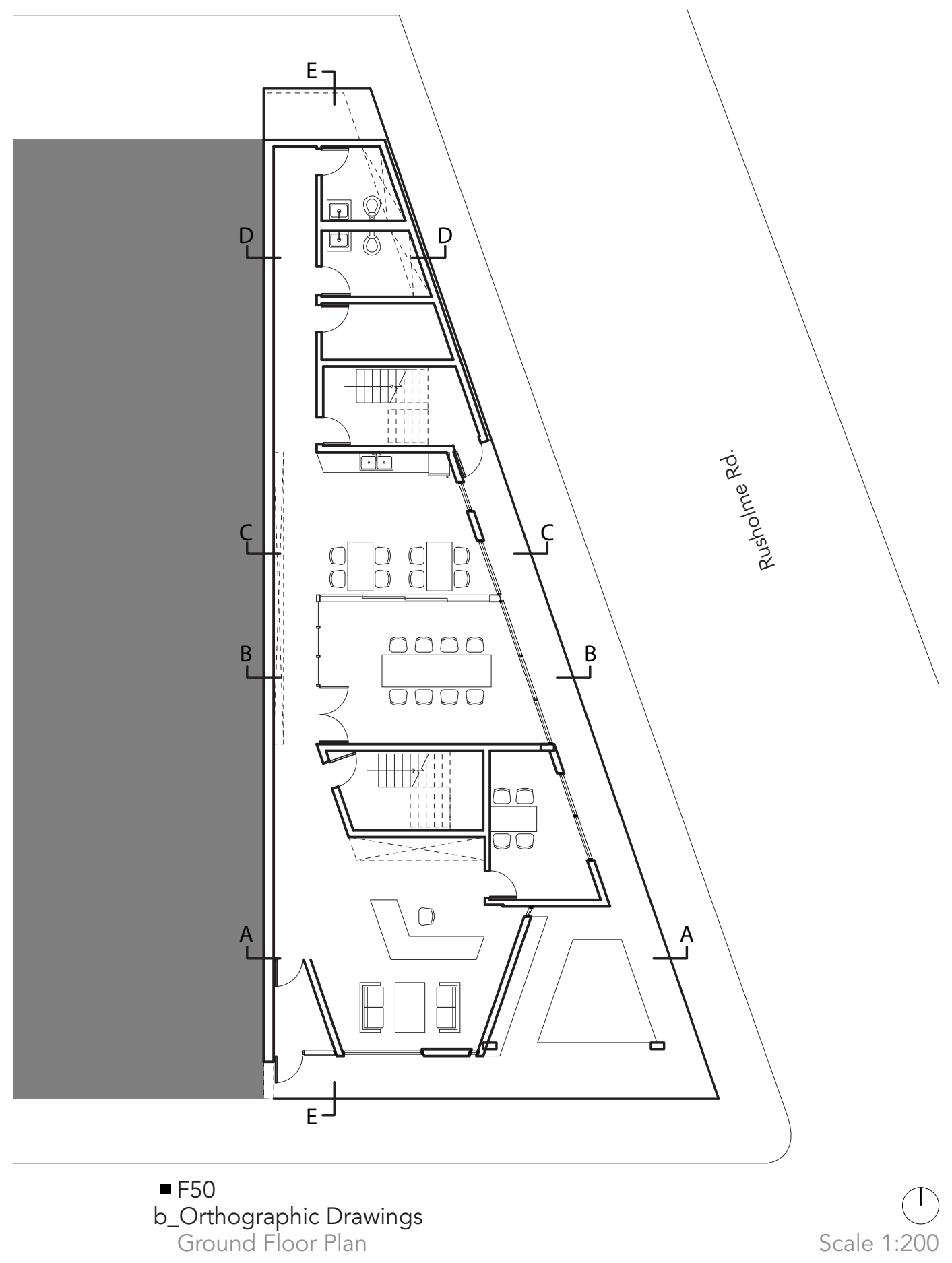

Light and Architecture | 75 


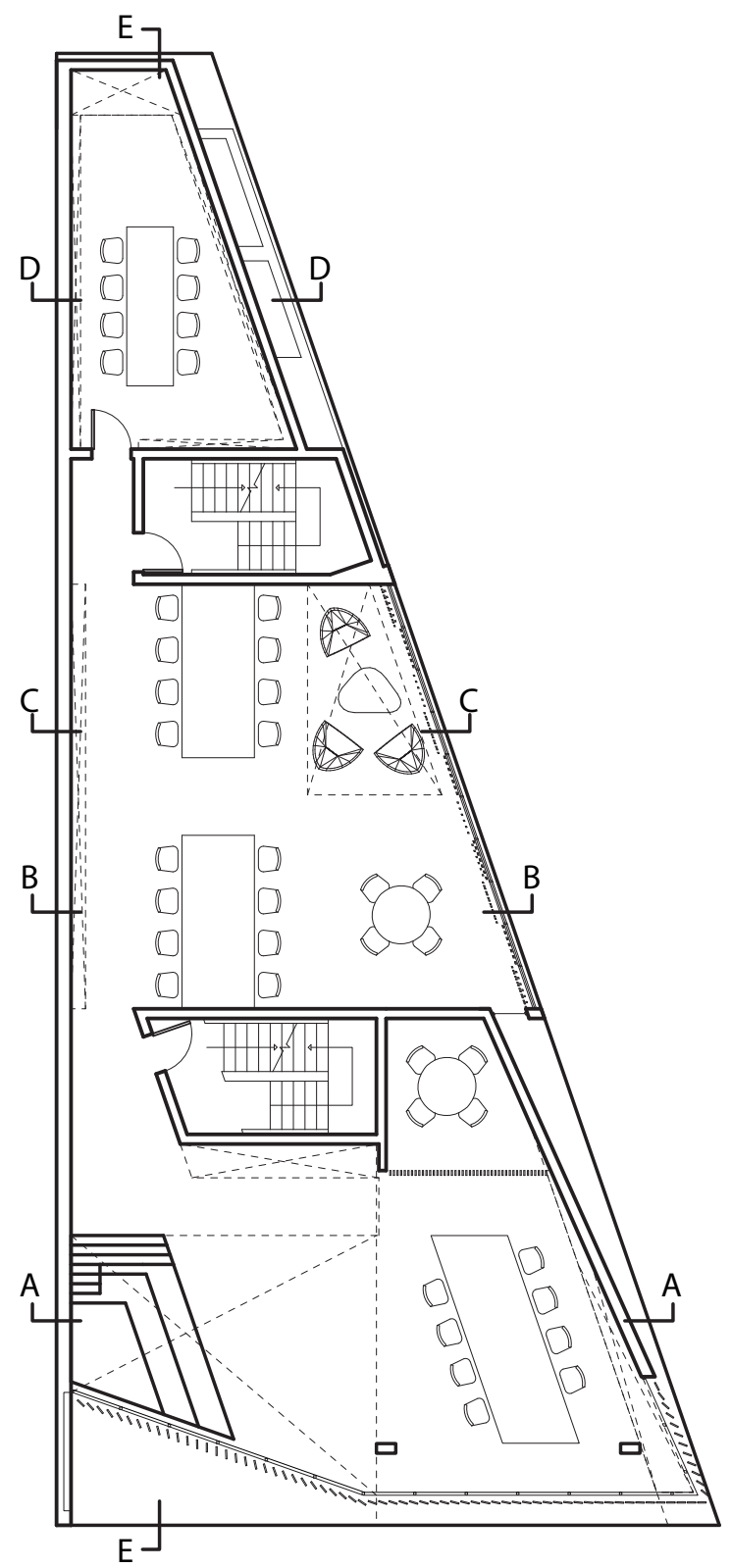

- F50

c_Orthographic Drawings 


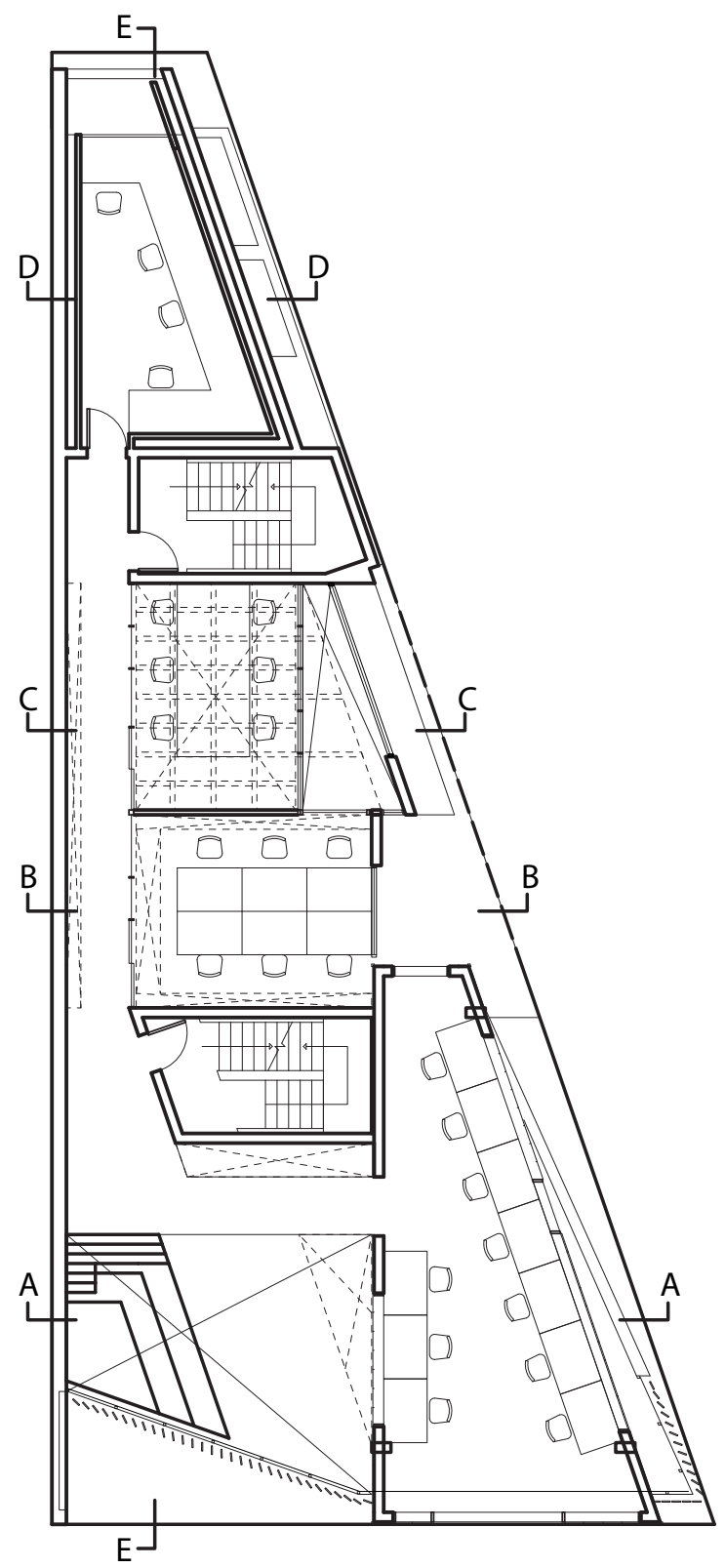

- F50

d_Orthographic Drawings 


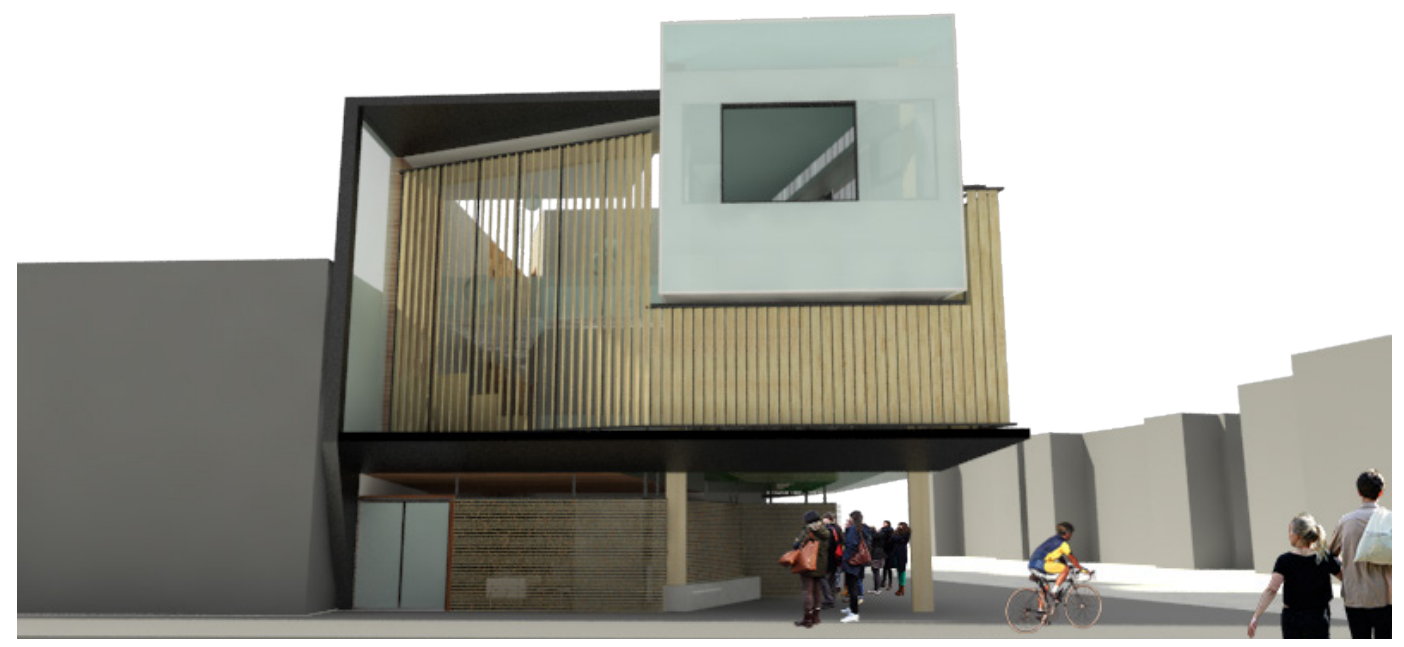

- F51 a_Exterior Perspective

The design of the office hub utilizes light, material and form to reveal the unique characteristics and design of the individual rooms. Within each space, a unique spatial ambiance and lighting condition are expressed to support the programmatic activity as well as create a spatial identity within the building. The spatial lighting condition throughout the building range from low light ambiance to highly illuminated space, supporting the functional requirement and experiential expression of light. Areas of high transparency provide indoor/outdoor connection (highly illuminated space), where opaque rooms create private and dark spatial environment. 


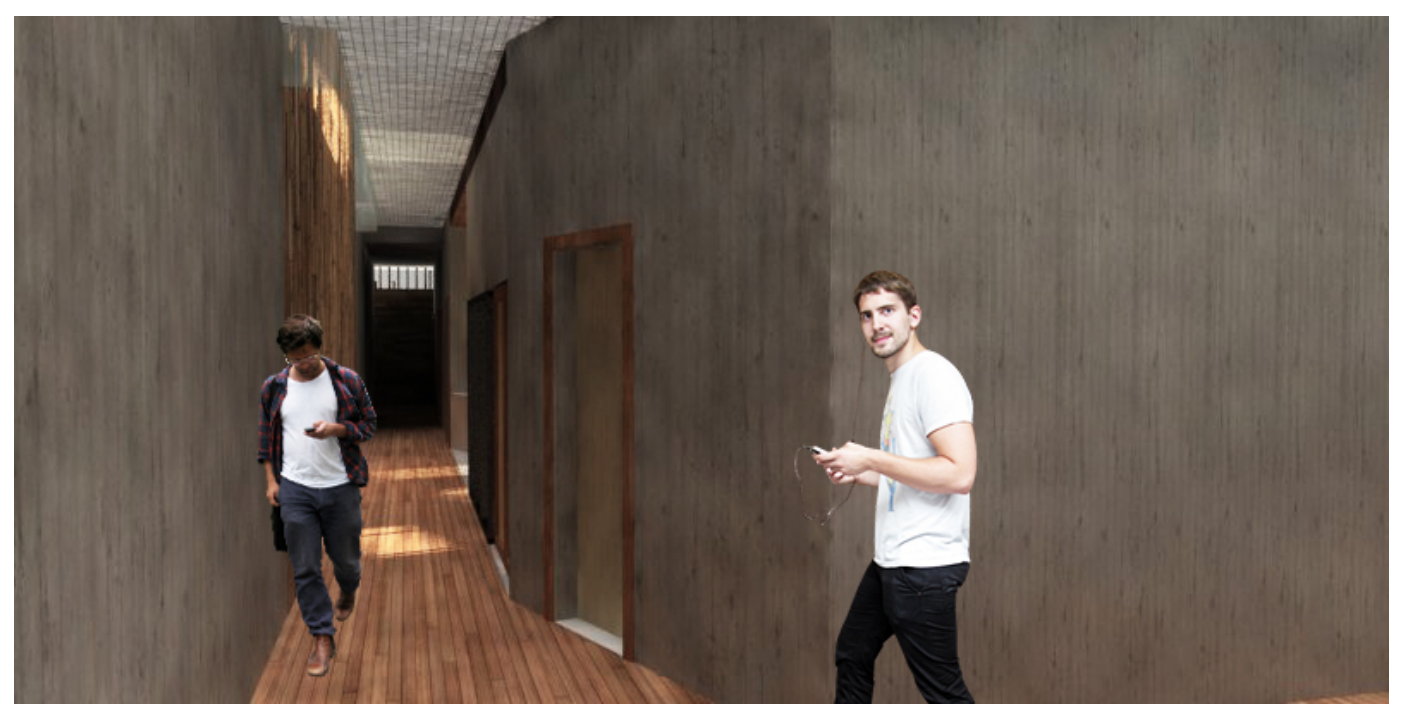

- F51 b_Hallway

The hallways utilizes material and form to integrate and express light. The use of a steel grate floor system allow ambient light to disperse between floors, making the space appear brighter and comfortable for circulation between offices. At specific time of the year (early noon), the designed light well allows sunlight to enter along the west wall. The illumination of wood slats of different depth create light and shadow variation display in the interior environment to provide perceptual stimulation for office workers.

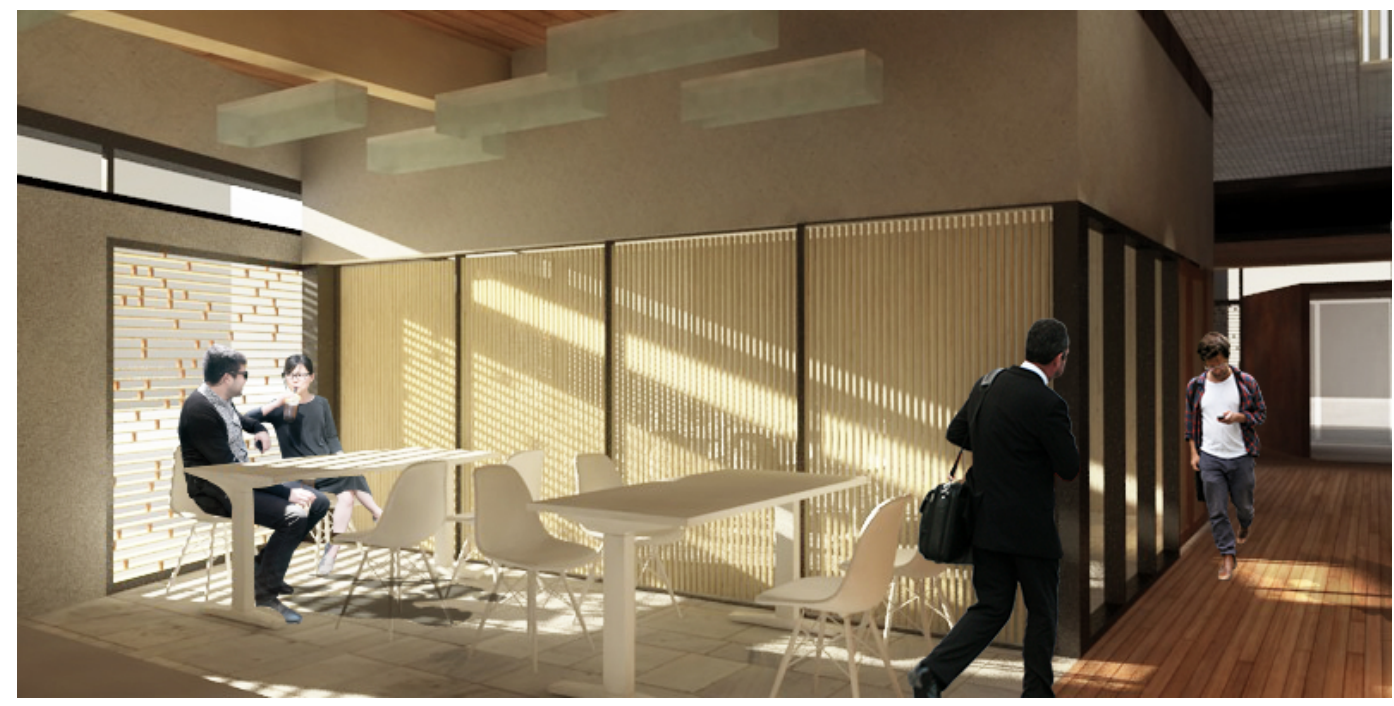

- F51 c_Kitchen

Located along the east façade, the kitchen is designed to stimulate the bodies of workers in the morning through direct solar exposure. The filtration of light through horizontal louvres softens the amount of direct solar exposure, creating a dynamic environment through the interplay of light and shadow. The integration of material, solar exposure and spatial ambiance define the characteristics of the kitchen in the morning. 


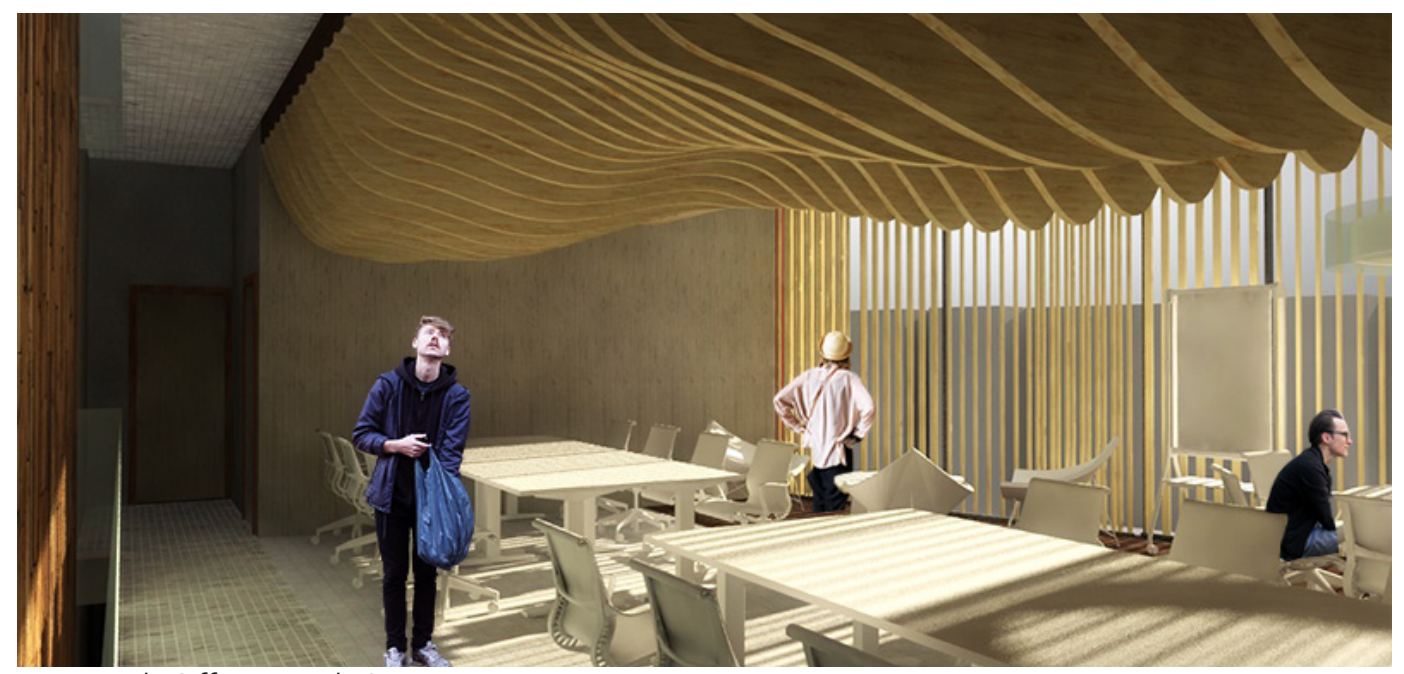

- F51 d_Office Work Station 1

Located along the hallway, the temporal workstation receives direct solar exposure during the morning. The filtration of light through vertical louvres ensure light is able to enter deep into the building while providing consistent illumination as it mediates between spaces. A sense of verticality and spatial compression is experienced within the space through the undulation of ceiling panels and vertical lines. The treatment of white ceramic tile allows more light to be reflected, creating a bright spatial ambiance for workers throughout the day.

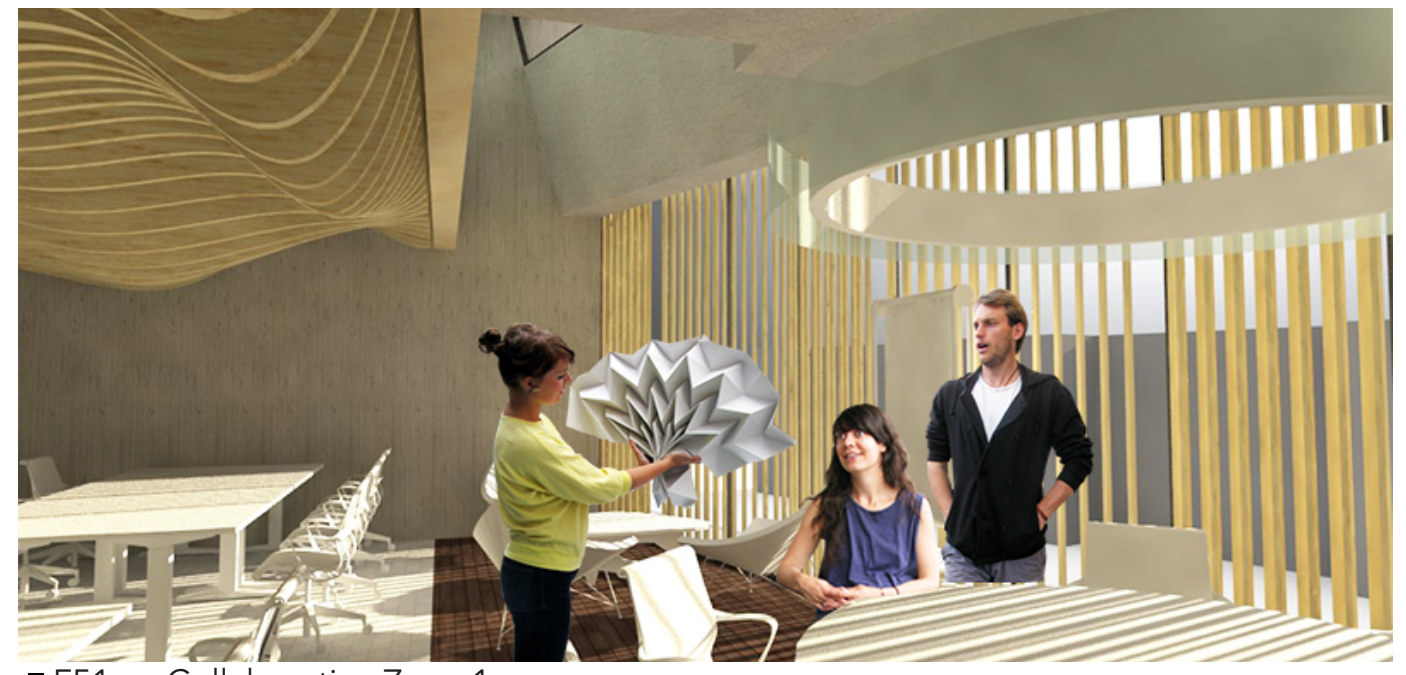

- F51 e_Collaborative Zone 1

The dark wood treatment along the floor reduces the amount of reflected light along the eastern window, contrasting with the white ceramic tiles. This dynamic collaborative zones introduces direct solar exposure during the morning, afternoon, and evening as it contacts walls and floors. The stucco treatment along the walls provide a textural surface to create light and shadow variation to enrich the architectural environment. 


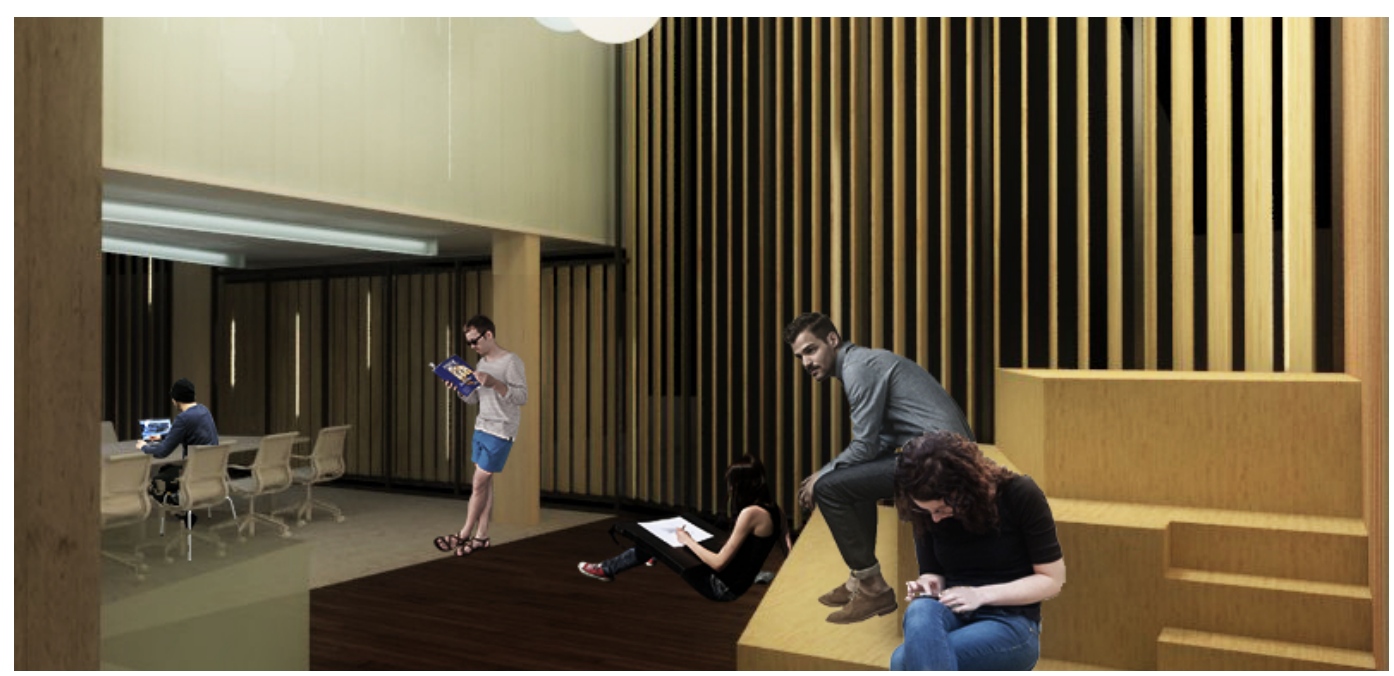

- F51 f_Collaborative Zone 2

Located on the south façade, the double height collaborative space is designed to appear open and highly saturated with light. Direct southern light exposure create dramatic light and shadow variation within the space, where it is designed perceptually stimulate users after long working hours. An open seating arrangement allows users to freely move around the space that suits their comfortable lighting exposure and condition. The transition from a bright area and dark zones is expressed through the rotation of the louvres.

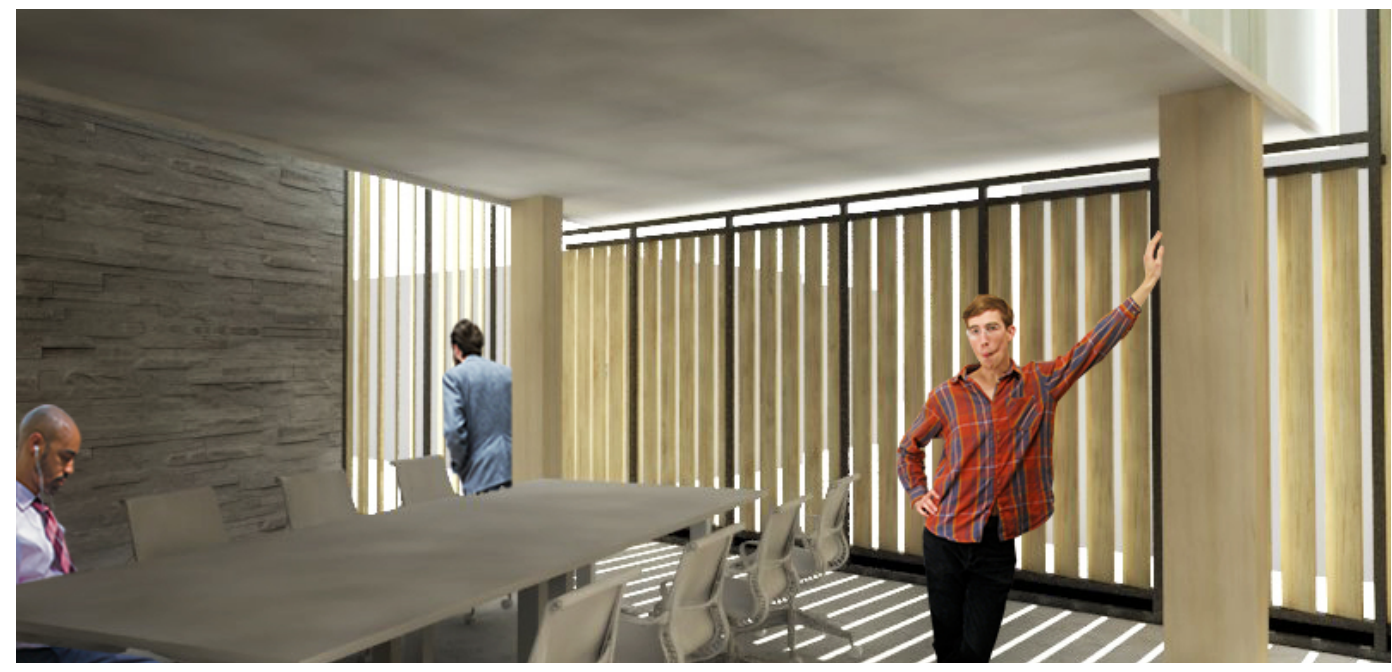

- F51 g_Office Work Station 2

The design workstation expresses a dark and sensorial environment as low light ambiance is introduced. Direct solar exposure are expressed and revealed along the walls and floors, reducing the distraction on workstation and providing indirect illumination within the space. The screening of light along the eastern façade in the morning, and the trickling of light along the interior white stonewall in the afternoon provides light and shadow stimulation that enriches the spatial experience. 


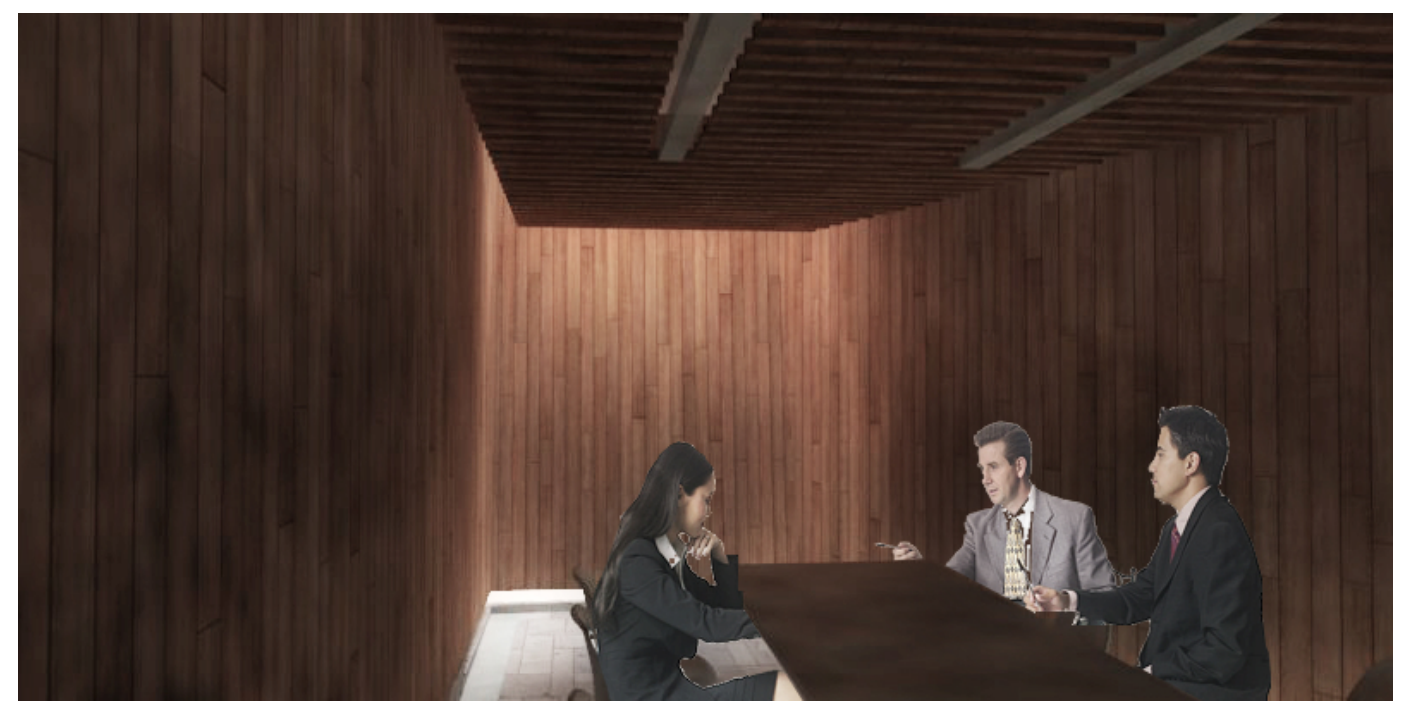

- F51 h_Private Meeting Room

A cave like spatial experience is perceived through the dark lighting condition and illumination from above. A sensorial environment is experienced within the meeting room as the low light ambiance desensitize the eye, mind, and body. The room caters to projection work and digital media as the illuminated work becomes the primary focus. Indirect illumination along the perimeter of the room reveals the textural characteristics of the dark wood treatment within.

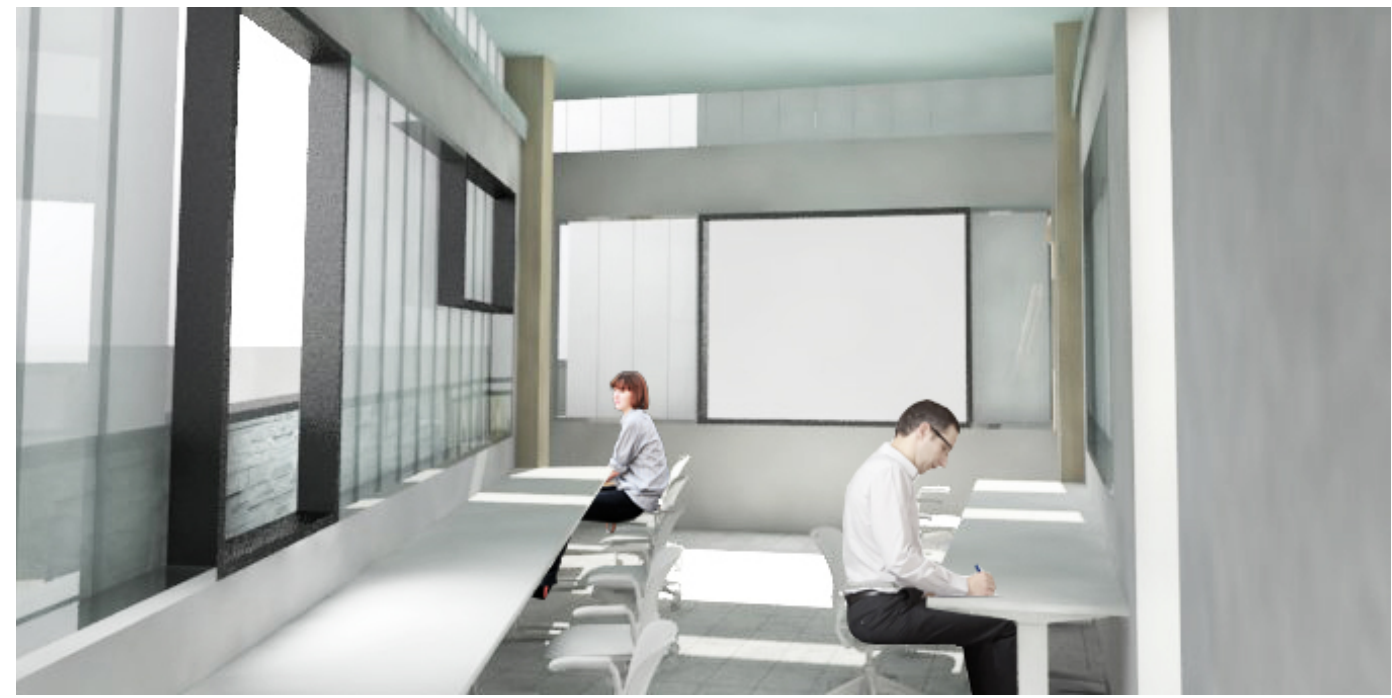

- F51 i_Office Work Space 3

The workstation is designed to receive a high amount illumination throughout the day, making the environment appear refreshing and energized with light. The use of channel glass wall system allows sunlight diffuse into the space, where at night it is able to emit a soft glow. Whether in the morning, afternoon, evening or midnight, the space is designed to allow workers to feel alert and awake. 


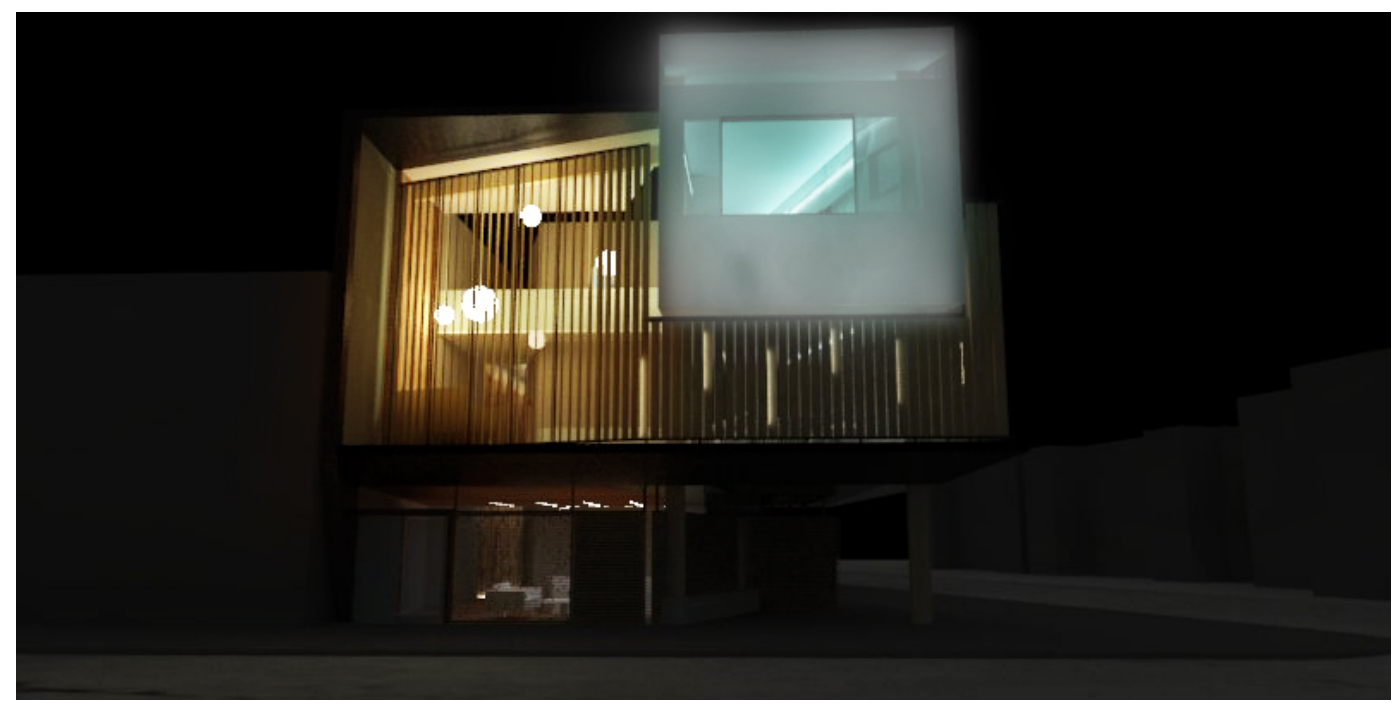

- F51 j_ Street Perspective

The south façade is designed to create two contrasting spatial environment that express and utilize light differently from one another. The collaborative space is designed to be dynamic, comforting and engaging through the use of material and light, providing and indoor and outdoor connectivity. Whereas the work station is designed to be private, with minimal visual connection. The materials used along the interior walls provide different hue of light and alter user perception and experience.

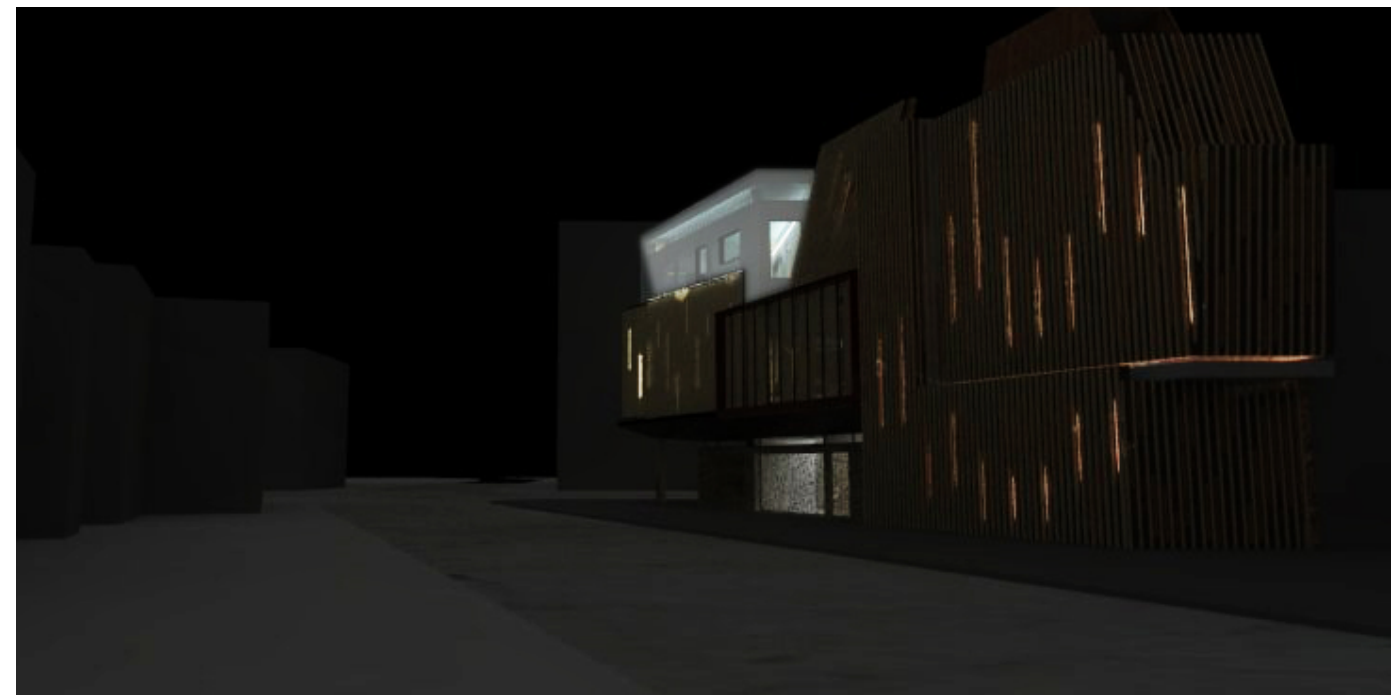

\section{- F51 k_Street Perspective}

The integration of artificial illumination along the exterior wood panels of the facade creatively utilize and display light along the urban street. Subtle variation in lighting organization and design provides perceptually unique and intriguing lighting effects. The design of the Eastern façade consists of uniquely designed individual forms that expresses and reveal light differently from one another. 


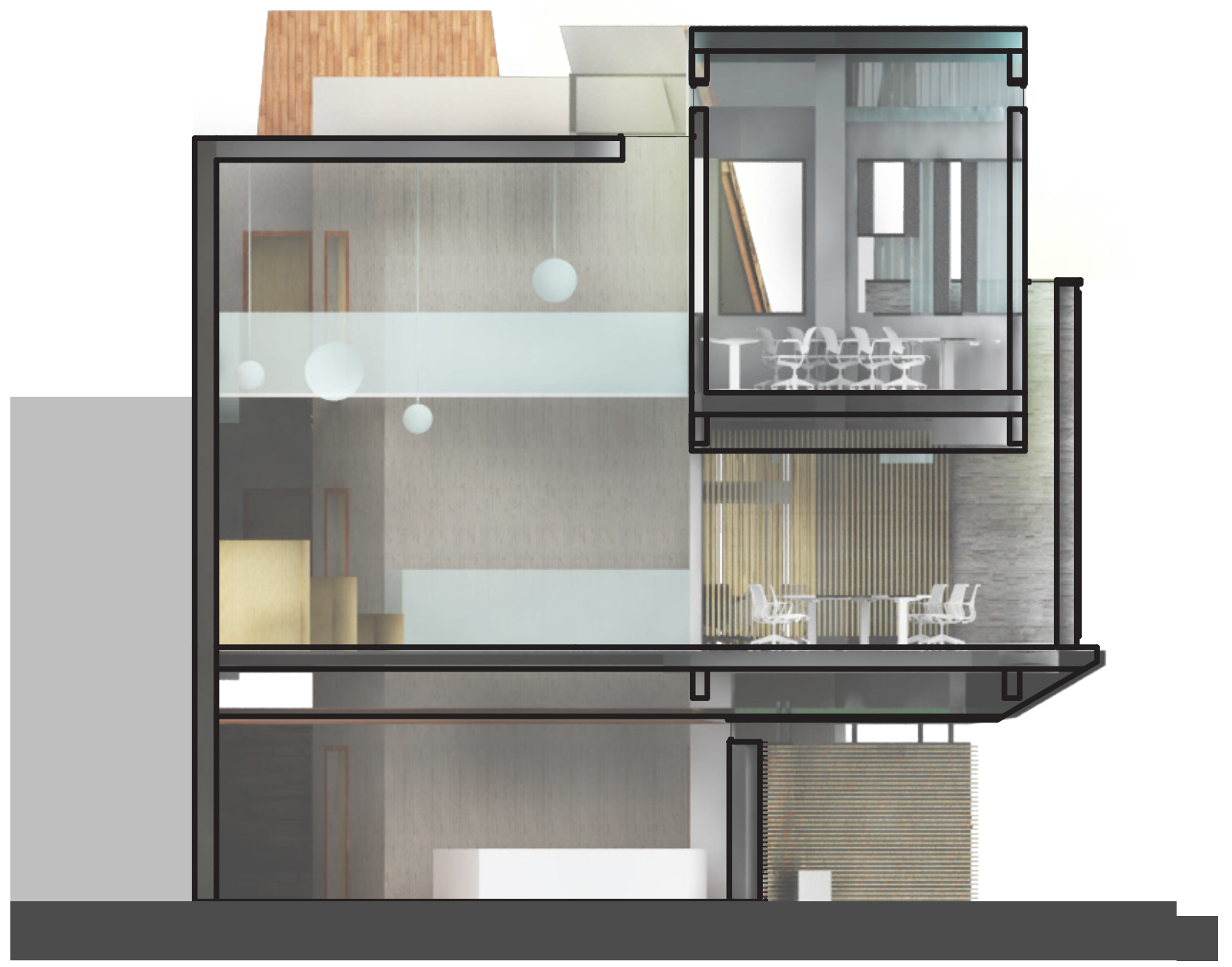

- F52

a_Orthographic Drawings

Section A-A

Scale 1:100

84 | Light and Shadow Expression 


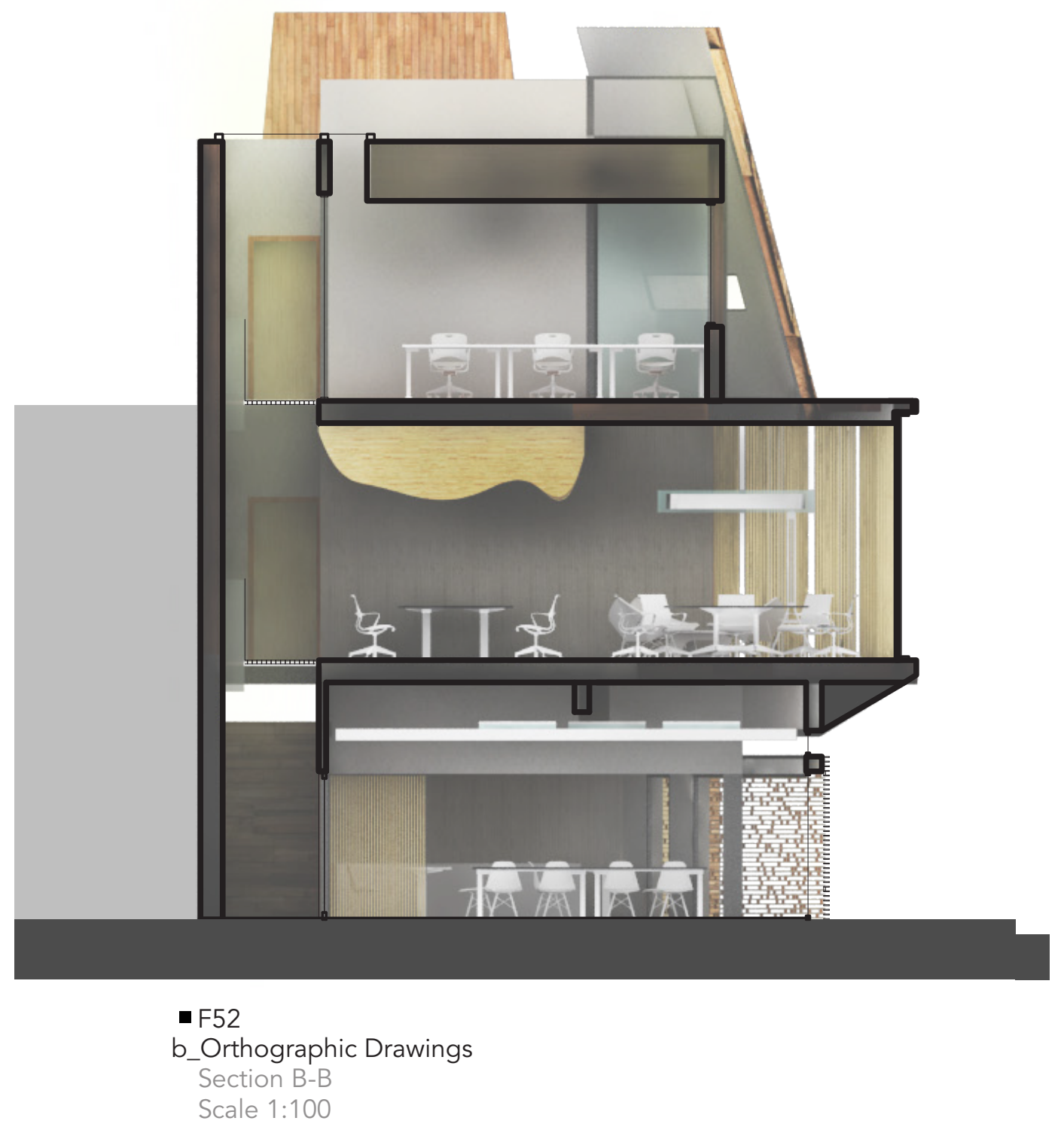

Light and Architecture | 85 


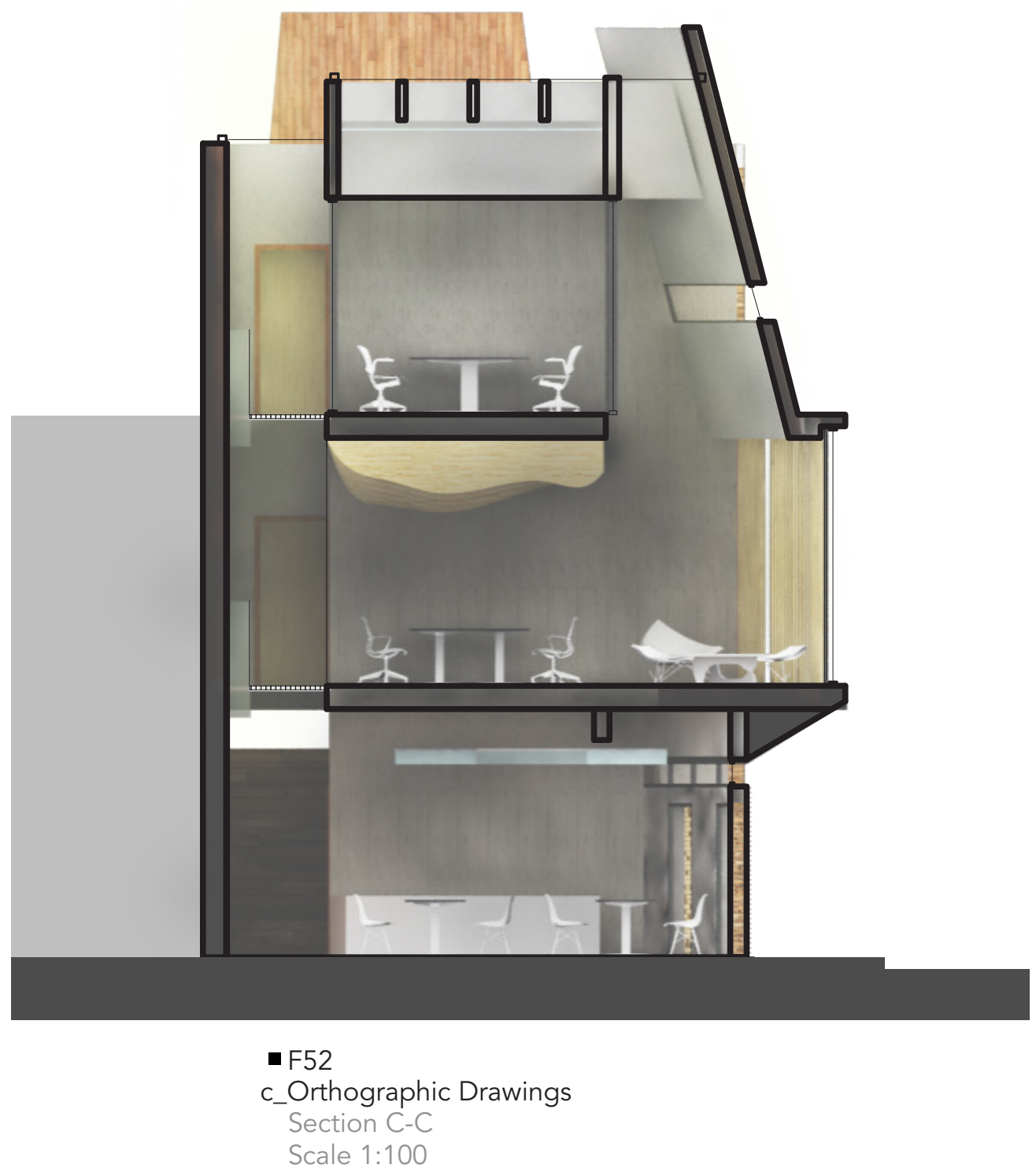

86 | Light and Shadow Expression 


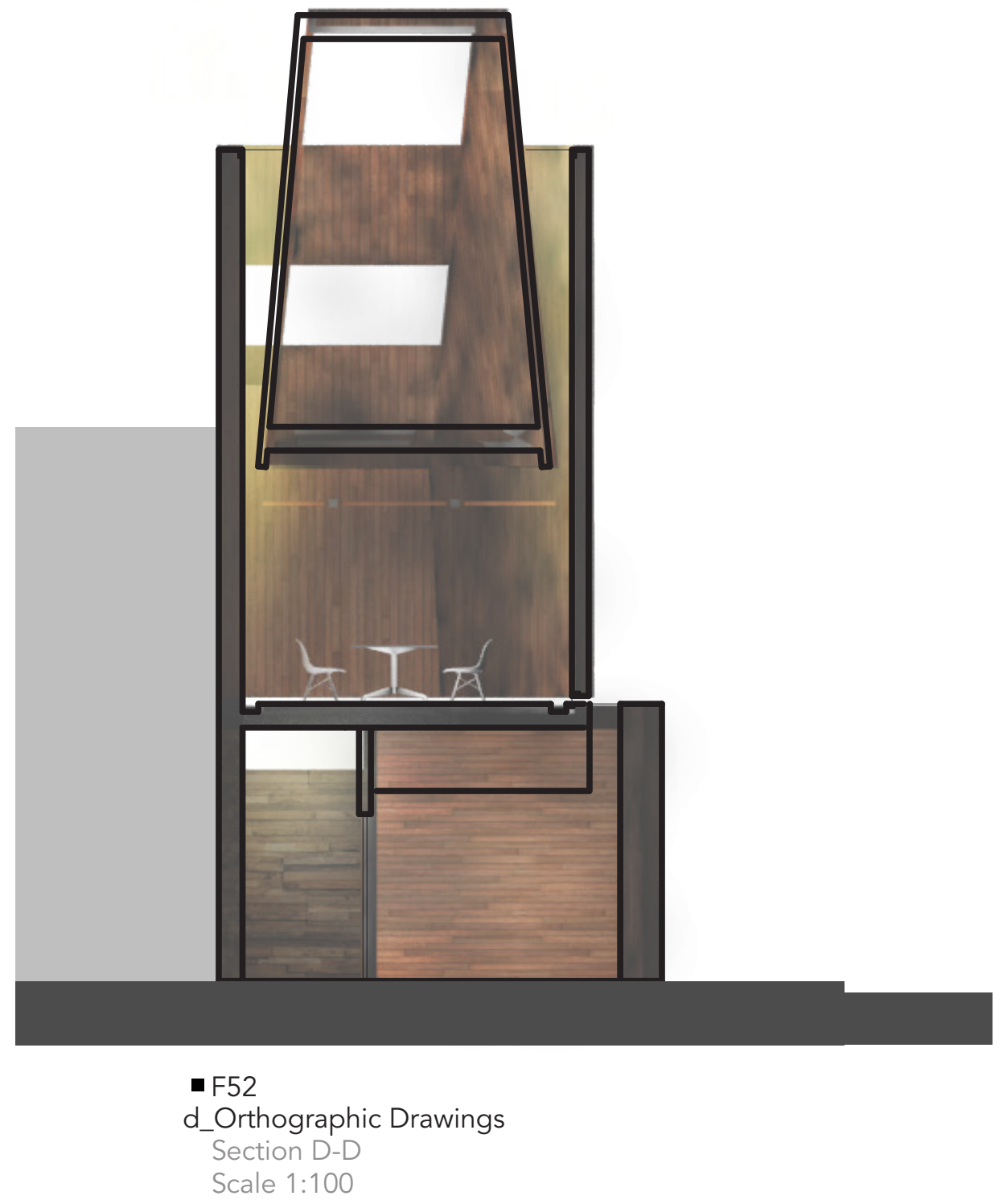

Light and Architecture | 87 

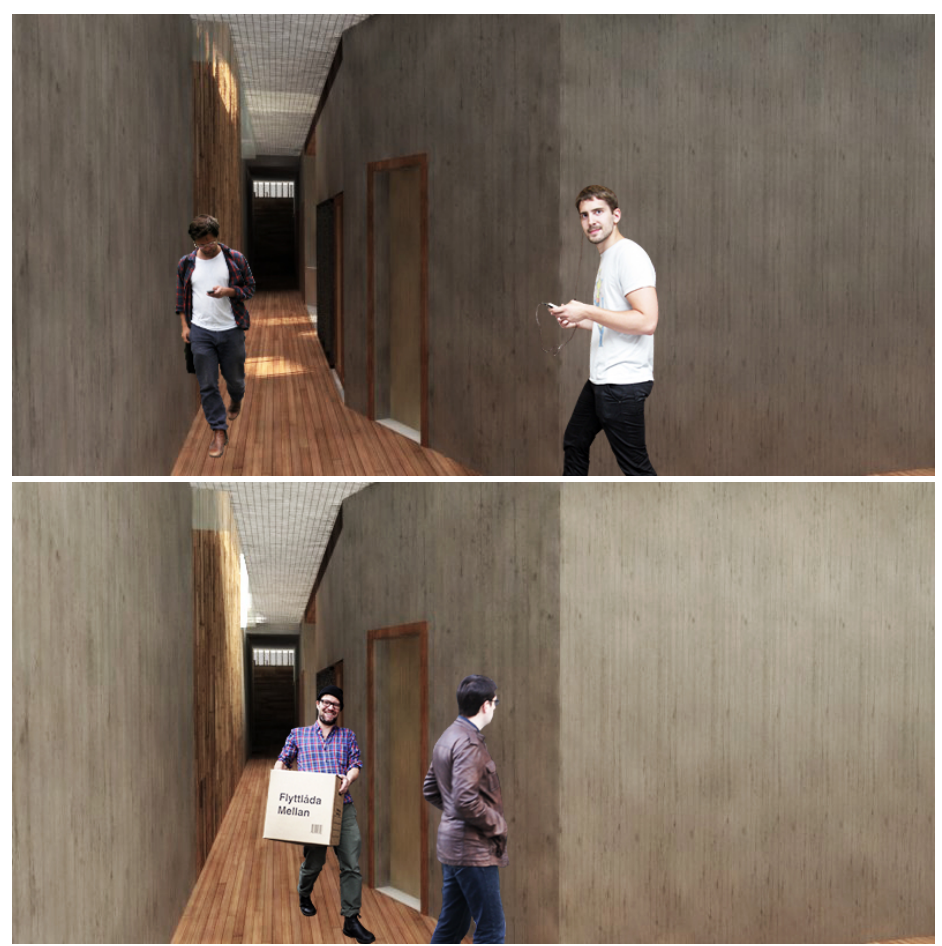

N
D
D
$\frac{1}{3}$
0
O
O

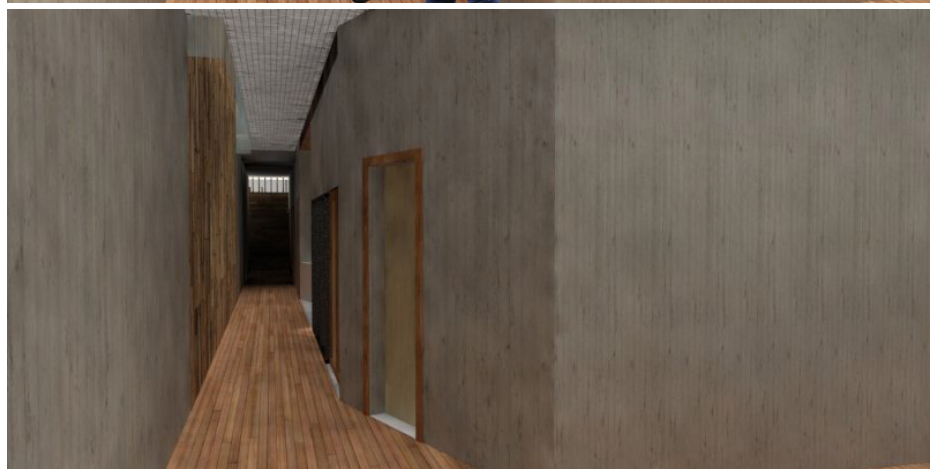

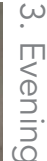

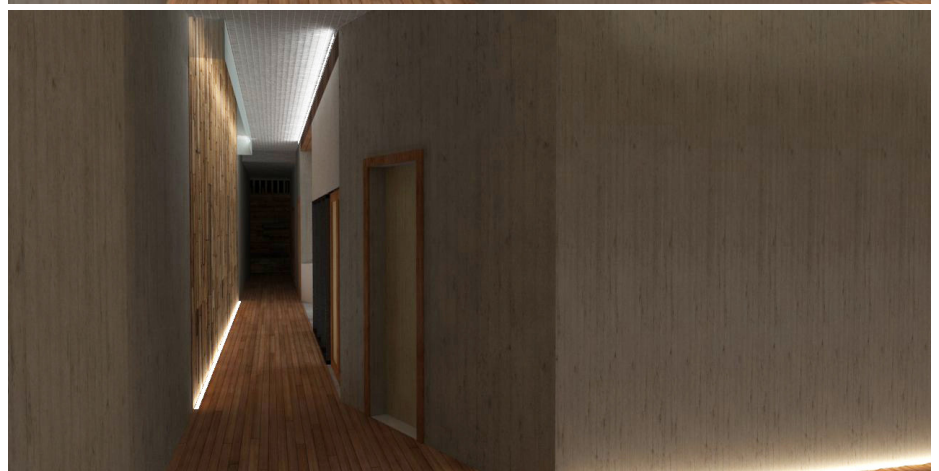

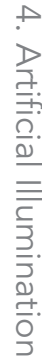

\section{- F53}

a_Hallway

88 | Light and Shadow Expression 

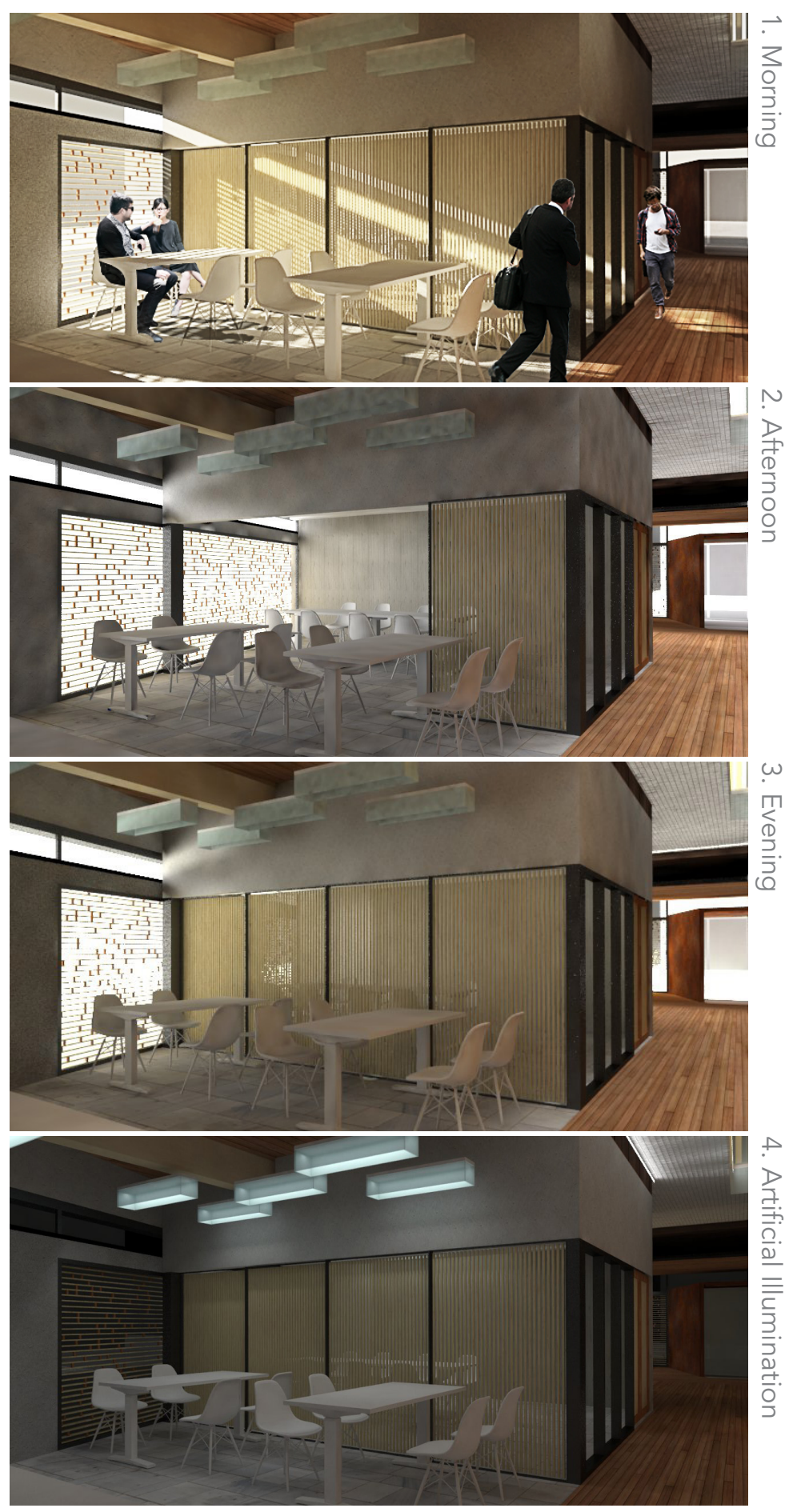

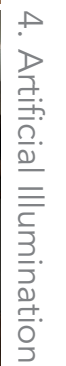

- F53

b_Kitchen

Light and Architecture | 89 

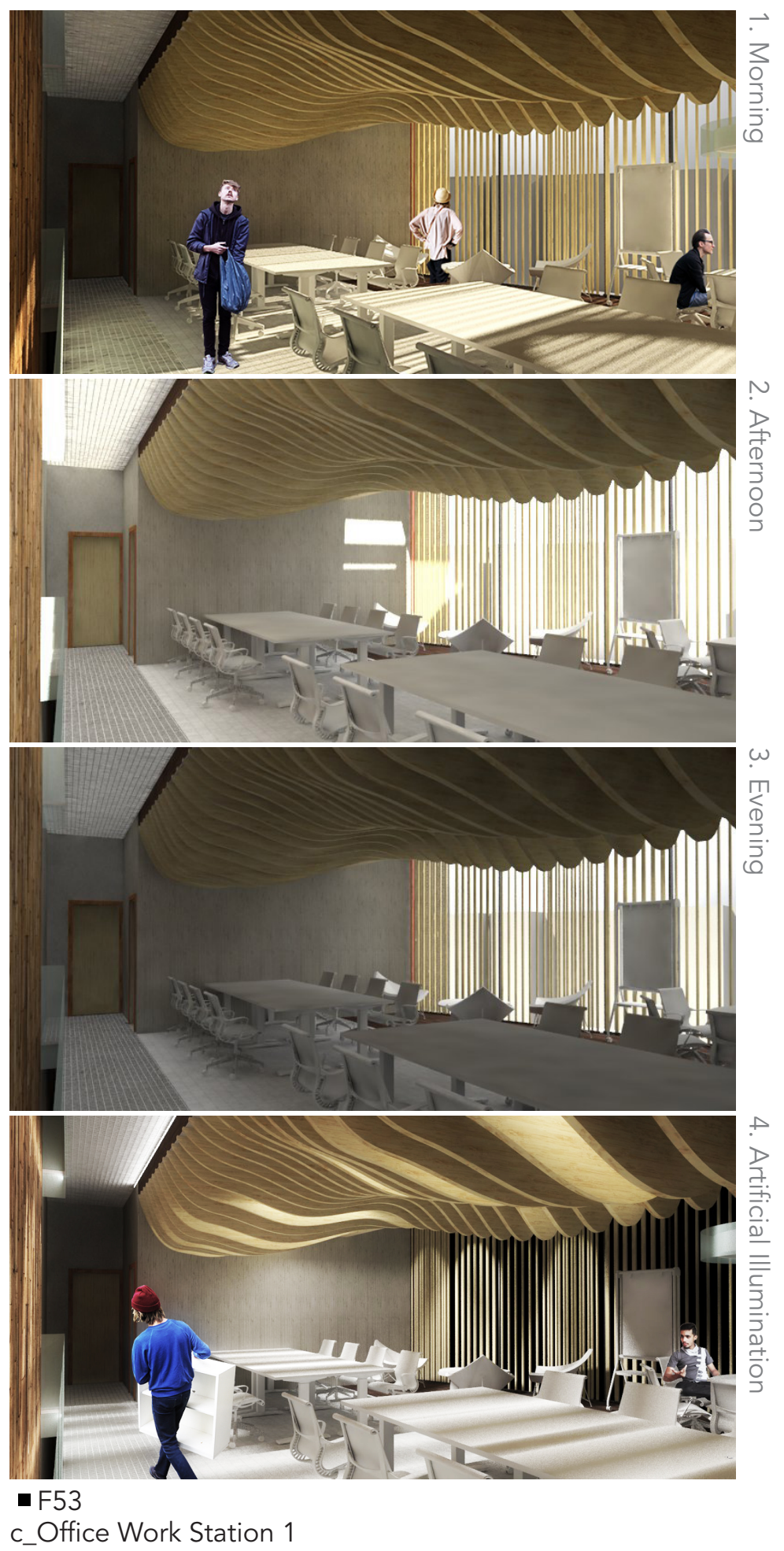

90 | Light and Shadow Expression 

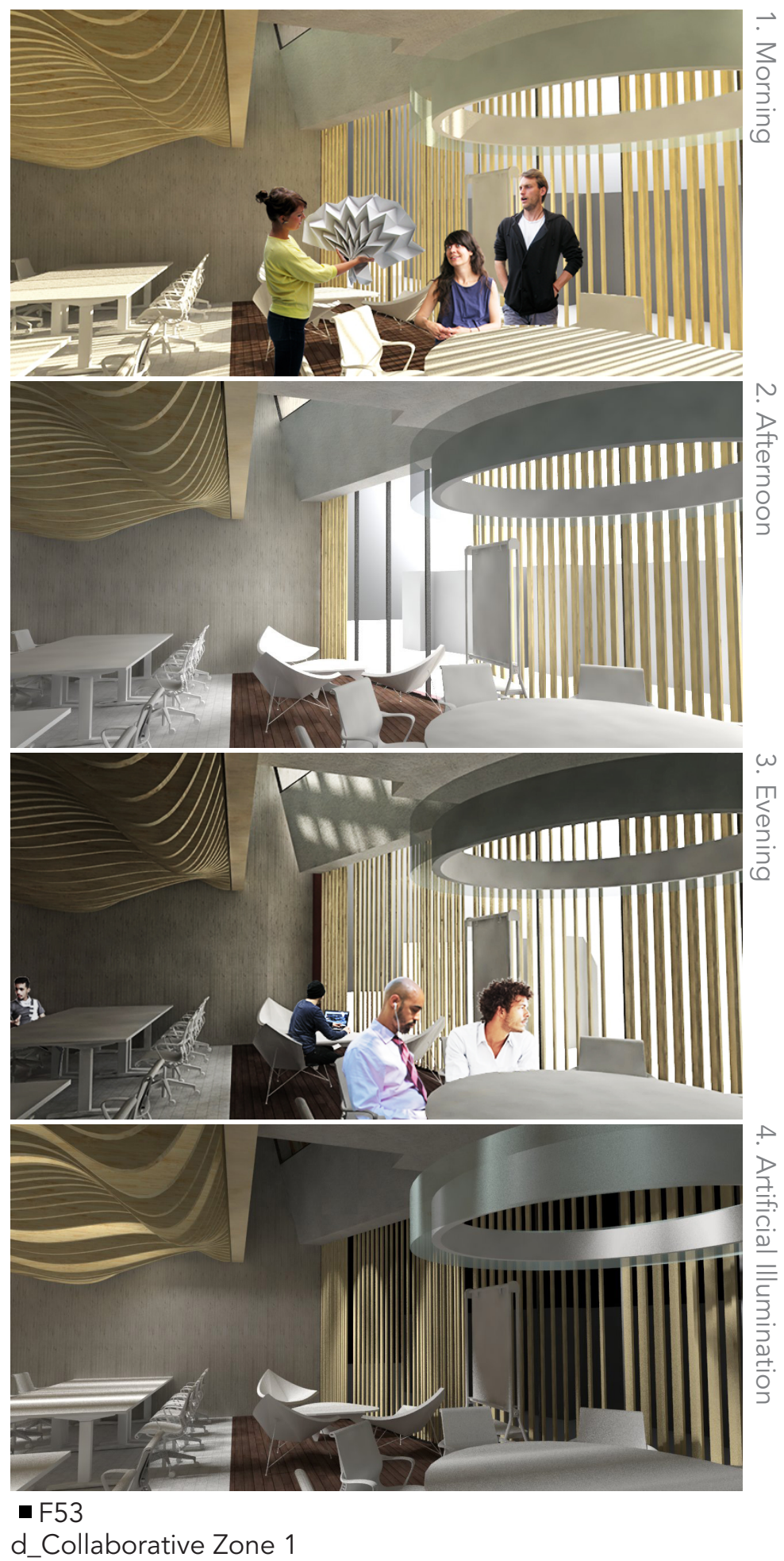

Light and Architecture | 91 

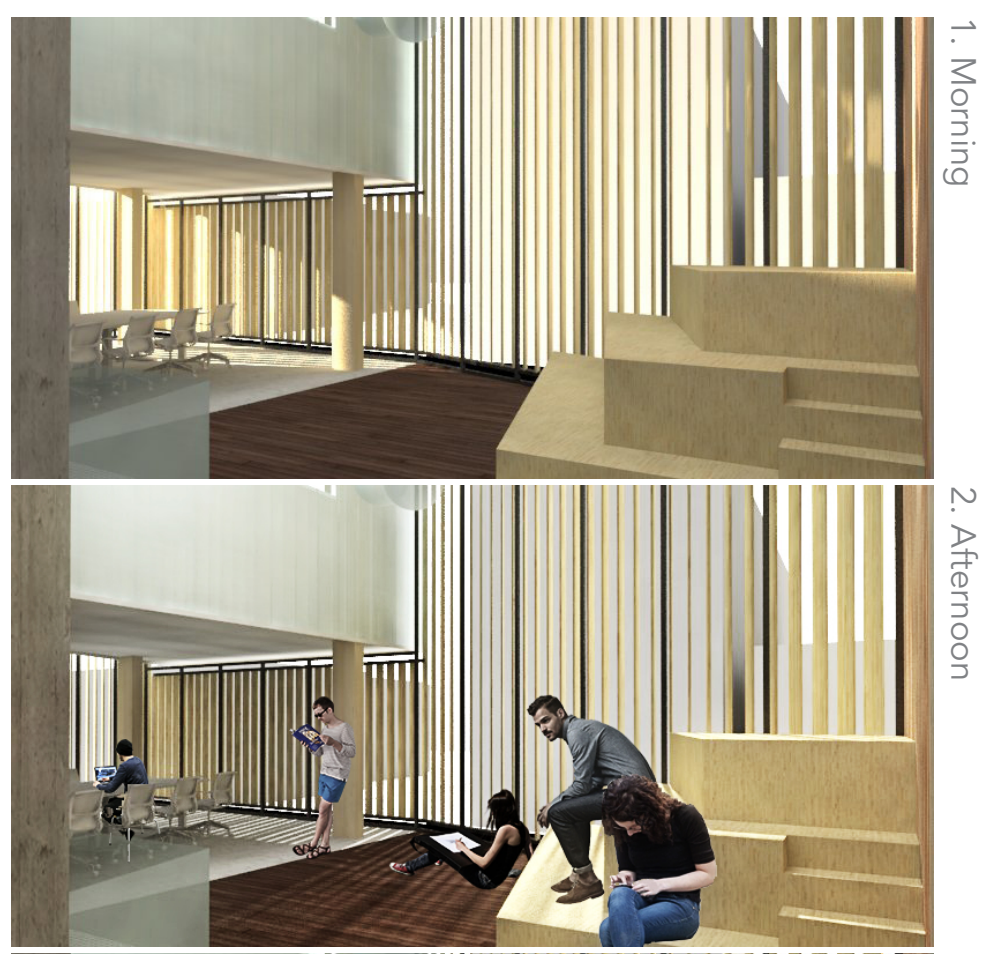

\begin{tabular}{l} 
N \\
D \\
市 \\
$\frac{D}{3}$ \\
0 \\
O \\
\hline
\end{tabular}
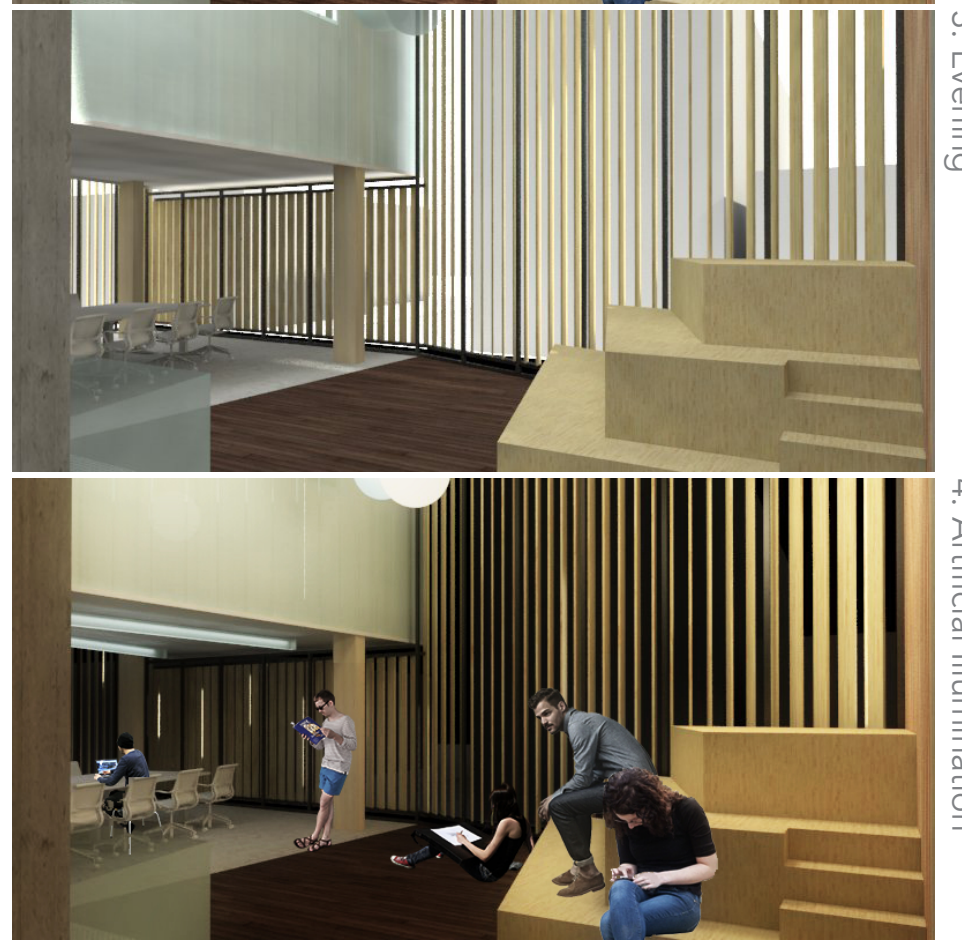

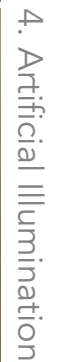

- F53

e_collaborative Zone 2

92 | Light and Shadow Expression 


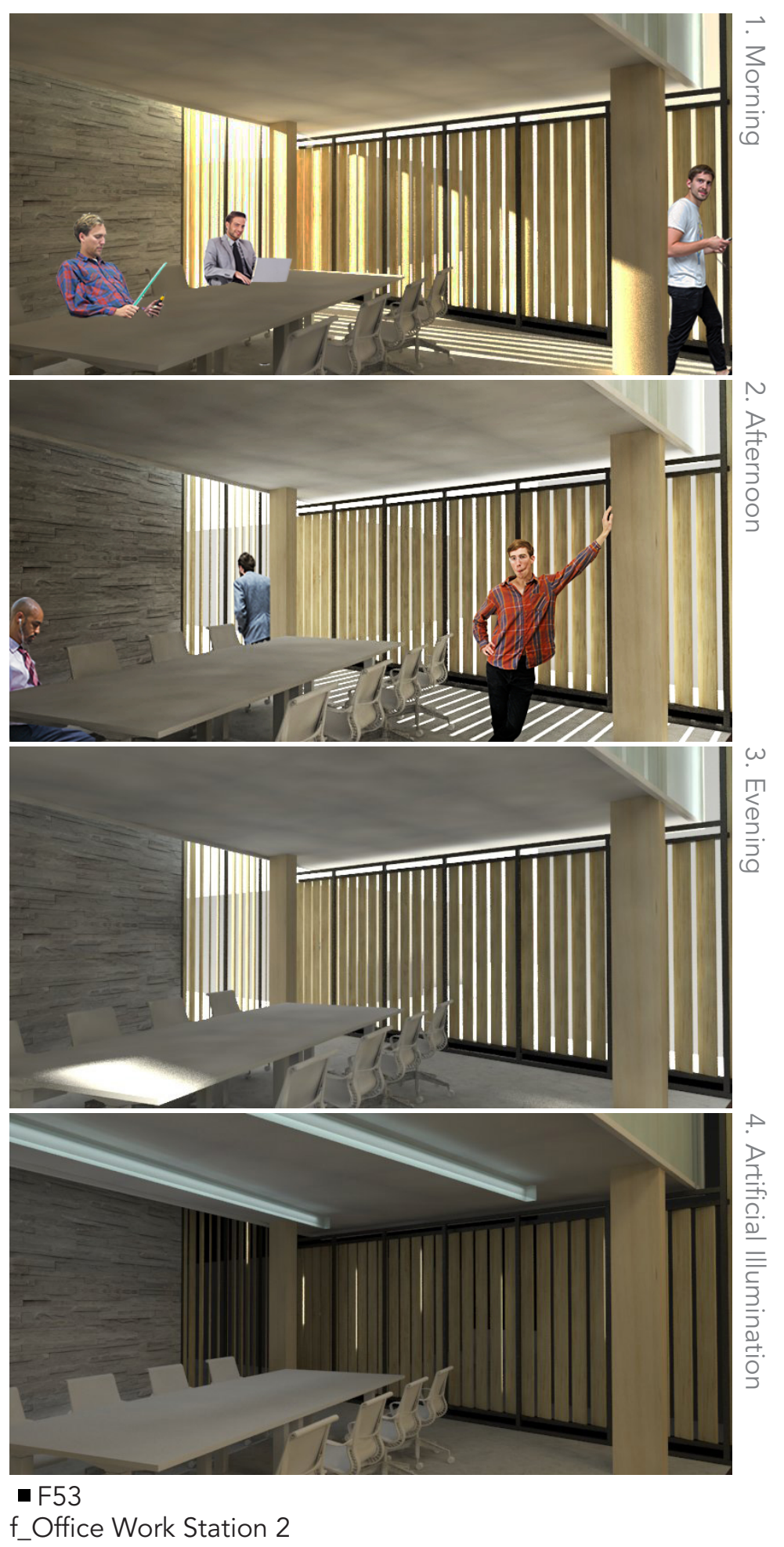

Light and Architecture | 93 


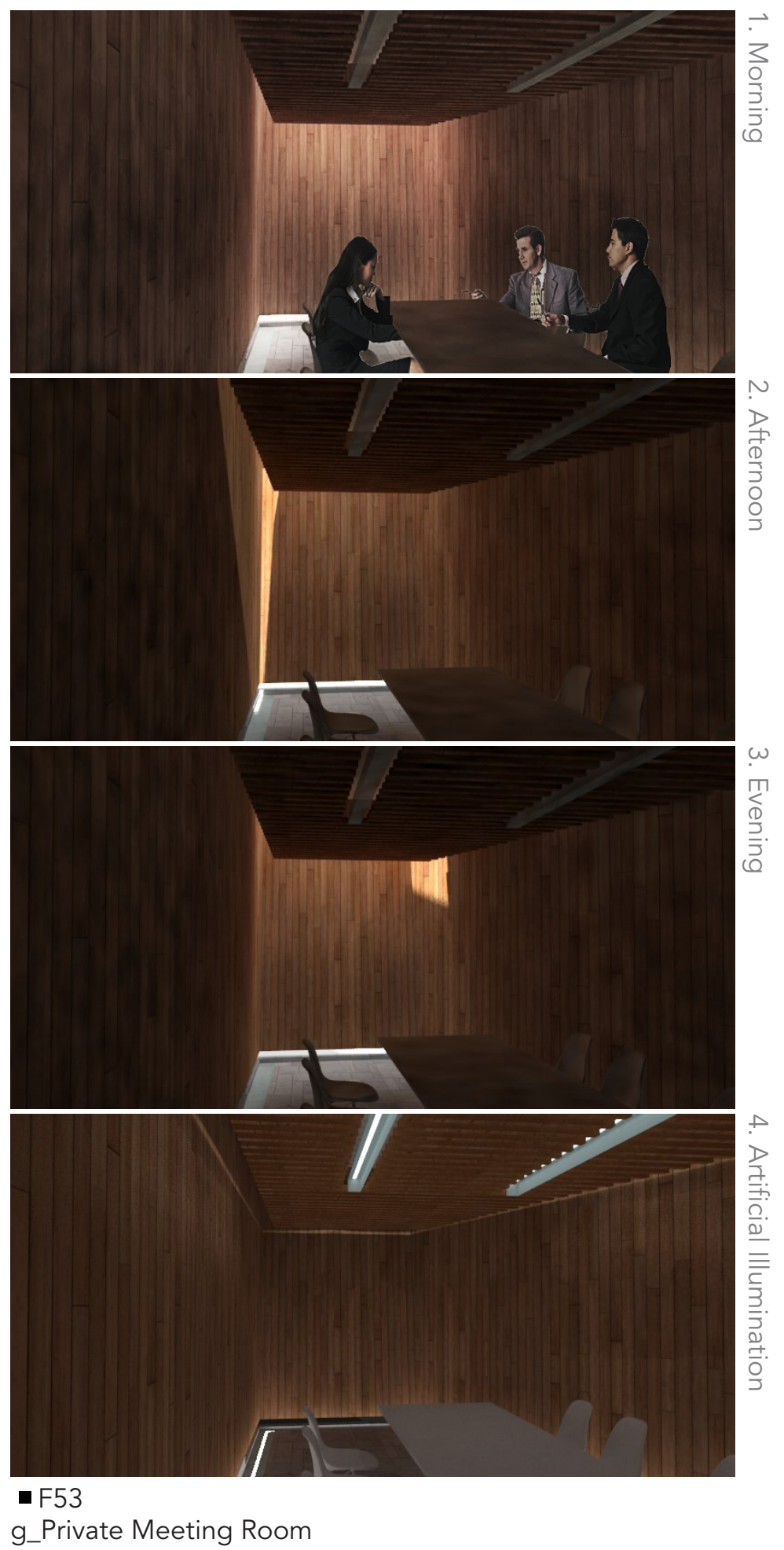

94 | Light and Shadow Expression 


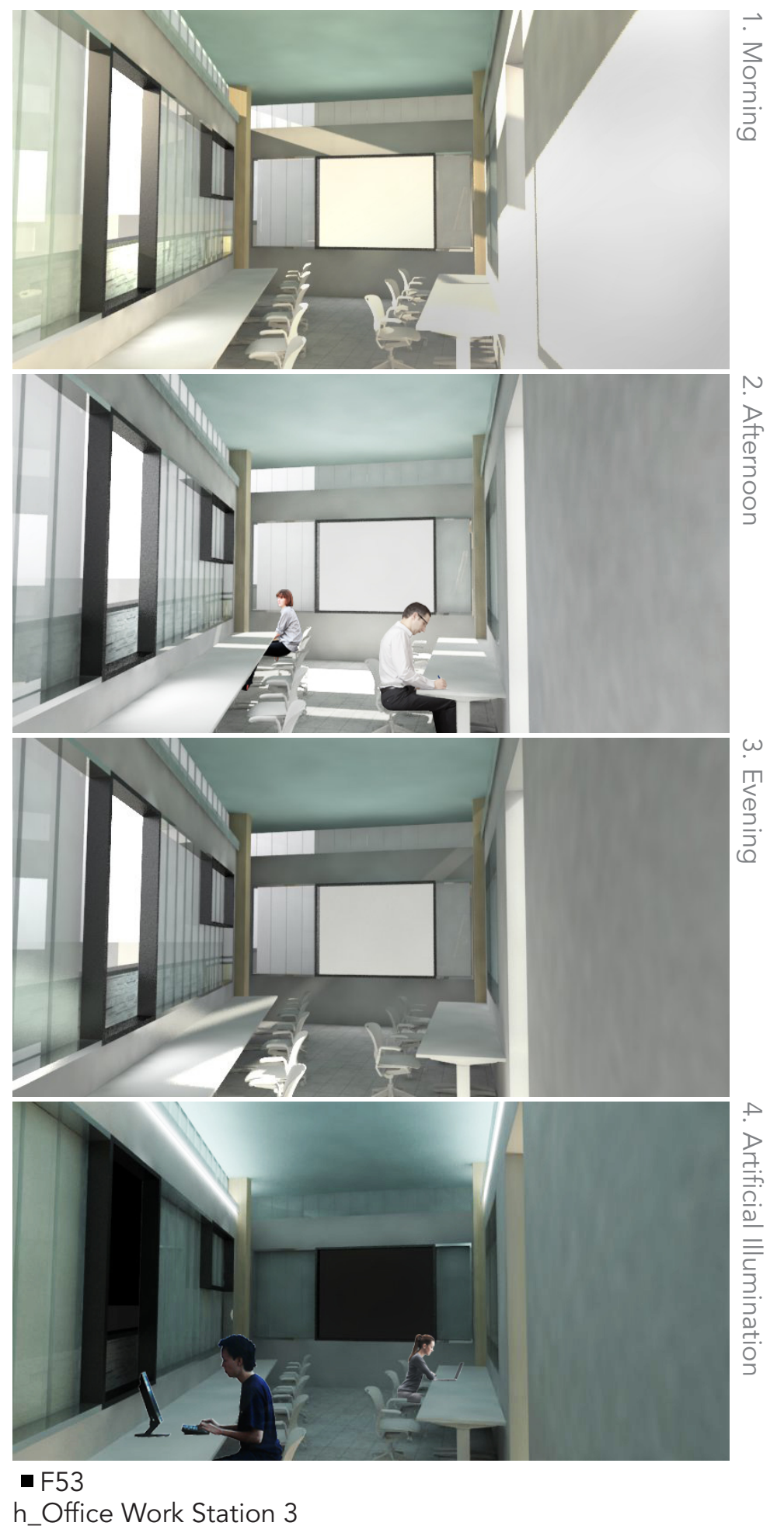

Light and Architecture | 95 


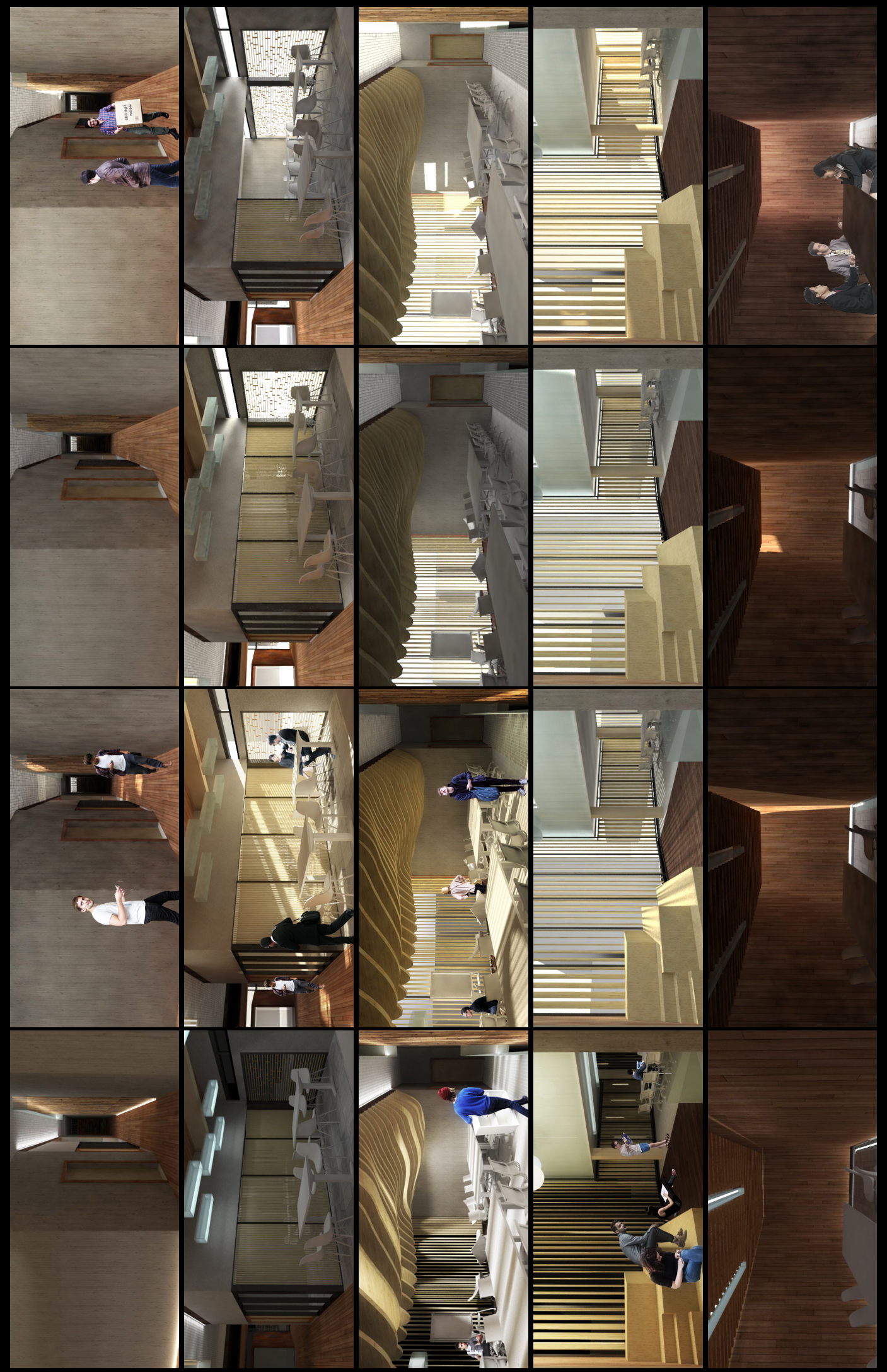

- F54

Rendering Compilation 

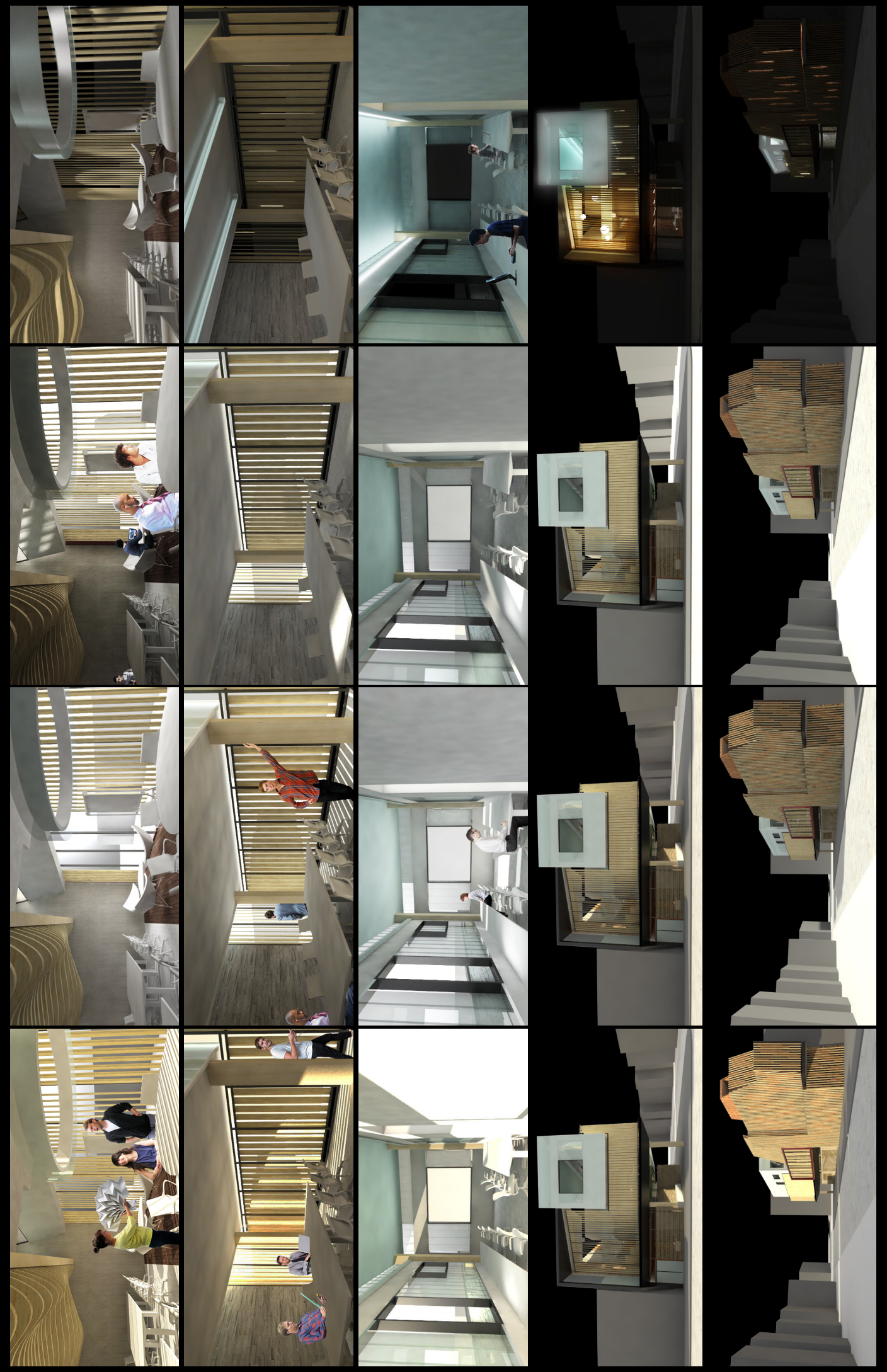


\section{Conclusion}

The expression of both light and shadow as a design medium enriches the characteristics and quality of space, providing architecture with perceptually fluid and stimulating experiences. The type of lighting a space receives influences the spatial activity, user experience, spatial ambiance and the characteristics of the built environment. A highly illuminated space is suggestive of an energetic, public, and social environment, whereas dark environments are describe as sensorial, intimate, and private spaces. Light provides a means of engaging the user's sensorial receptors, perceptually transforming the architectural experience as one moves through space. The dynamic characteristics of light as it changes throughout the day offers a design potential for architecture to express, creating temporal environment that are visually engaging and stimulating at specific moments in time. The expression of light is able to transform mundane architectural environments into sensorial, experiential and perceptually engaging spaces.

The evaluation of architectural lighting design through quantifiable design metrics examines lights functional role of spatial illumination and energy, whereas qualitative design aspects express its perceptual and experiential characteristics in shaping architectural environment. The evaluation of lighting design through codes and standards has provided architecturally homogeneous lighting 
conditions, depriving users of the architectural experience. The interplay of light and shadow offers architectural environments with visually diverse and experiential environments that provides users with a range of sensorial stimulus, engaging the eye, mind and body. Light reveals the characteristics and quality of material, form, texture, and colour, whereas darkness withdraws these feature providing space with a sense of mystery and ambiguity. The expression of light and shadow introduces experiential depth as the architectural environments perceptually transform throughout the day and night.

The pervasive use of glass curtain wall and artificial illumination has saturated architecture with light, depriving users the experience and expression of darkness. The use of sun filtering devices such as screens and louvres allow for the filtration and mediation of light between exterior and interior environment. The type of lighting and shadow effects produced correlates to its assembly and composition, where the size, shape, scale, and spacing of opening alters the type of lighting effects and experienced produced. Lighting variability and contrast are means of creating perceptually engaging architectural environments, providing users with an array of lighting stimulus and experiences within a space. 
Contemporary society has grown into a 24-hour cycle, where the expression of both daylight and artificial illumination introduces new experiential opportunity and spatial activity throughout the day. While the behaviour of both sunlight and artificial light are similar, its role within architecture differ from one another. Sunlight illuminates the interior environments, offering an artistic and experiential design expression within. Whereas at night, artificial illumination is able perceptually transform the built environment into illuminated objects, revealing the interior to the urban street. The design expression of both light and artificial illumination offers the potential to redefine user experience and perception.

Throughout history, religious buildings, museums and galleries have creatively expressed light in shaping the architectural environment and user experience. Whereas light's role in shaping the office environment lack the poetic and creative design expression. The organization of artificial illumination and windows in a grid like fashion produced homogeneous environments that deprive users of visual lighting stimulus. The integration of light has a significant role in improving the overall user well-being and experience within an office, transforming the mundane environment intro perceptual fluid spaces. The treatment of architectural lighting should differ from space to space, function to function, providing environments that are perceptually engaging and stimulating environments within architecture.

The integration of light and shadow has an important role in shaping the architectural environment and experience. Architectural lighting requires a creative design expression that influences the overall characteristics and quality of space as well as the users within. The design strategies listed within the thesis expresses light and shadow as a creative design medium to support and generate architectural spaces. Utilizing solar orientation as a method to generate the architectural experience enables the built 
environment to transform into a perceptually fluid environment, enriching the architectural experience and characteristics of space. The absence of the sun enables artificial illumination to command for attention, perceptually transforming the built environment during the evening. The integration of both solar orientation and artificial illumination provides unique experiential moments through lighting effects, altering the way users experience and perceive architecture.

The design of the architectural form in relation to the type of lighting effects produce allows for creative design integration and expression of light that enriches the characteristics and quality of space. The use of material properties, surface treatment and composition enables users to experience and reveal the unique characteristics of light as it interacts with material. The expression of light and shadow through texture and material provide lighting variation that engages the users' perception and enriches the quality of space. Lastly, the design of architectural spaces that provoke a particular ambiance through light provides a distinctive sensorial stimulation and experience. The type of lighting a space receives is able to drastically change the spatial functioning and experience within architecture. The strategies outline reinforce the importance of utilizing light and shadow as a design expression to generate architectural environments. The interplay of light and shadow allows for perceptual stimulation that enriches the architectural experience and environment. 
102 | Light and Shadow Expression 


\section{Appendix A - Light/Shadow Experiments}

The experiments conducted reveal the potential of light and shadow expression to generate architectural environments and experience. The abstraction of these lighting design concept models provide opportunity to apply within the design of architecture. The characteristics of material, texture, form, shape, space, etc. are method to express and experience light to generator the architectural environment. Lights integration within the built environment is able to perceptually transform spaces, changing the characteristics and perception of its surrounding. 

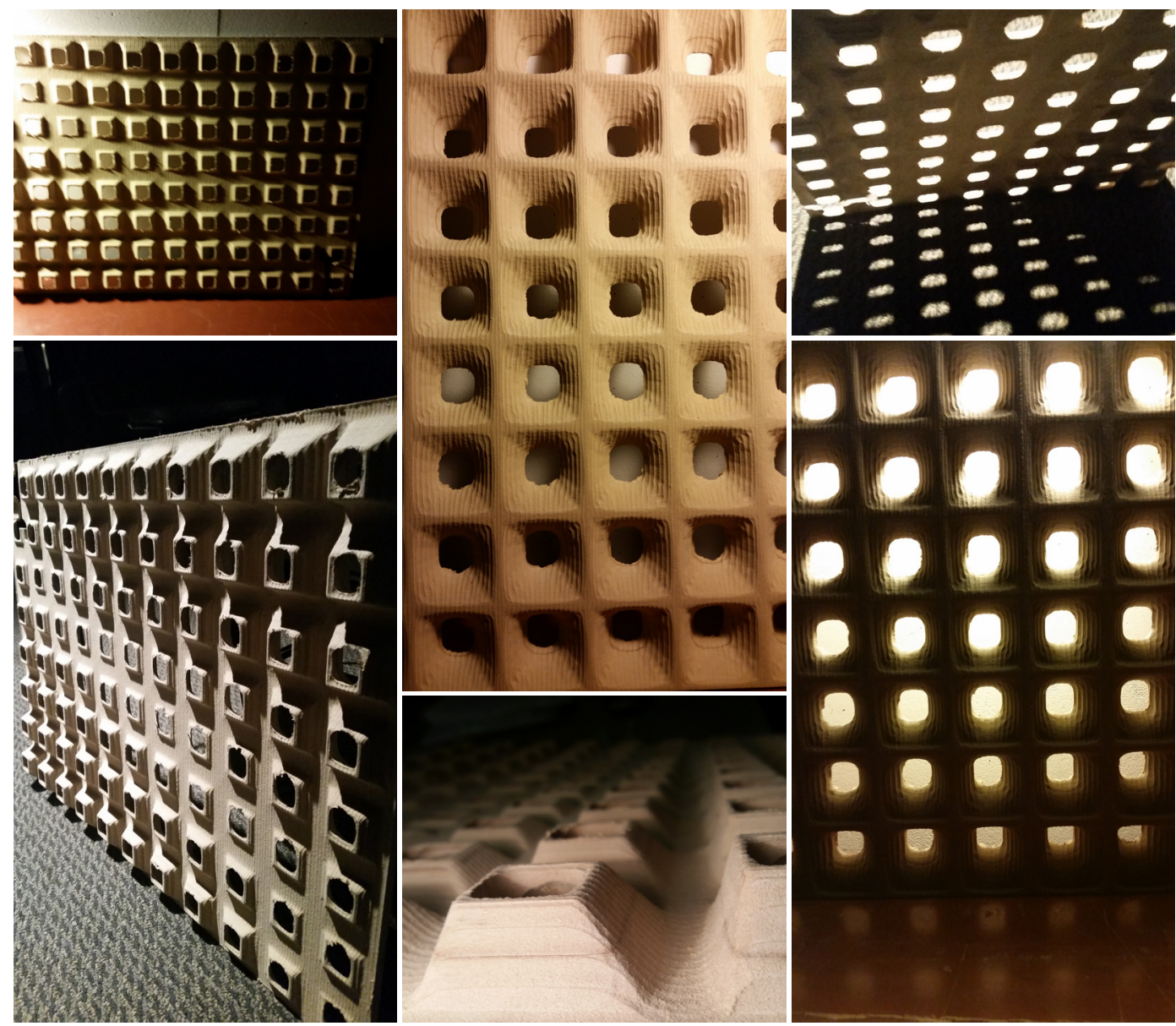

- F55

CNC Apertures Experiment

The designed experiment examines the expression of light and shadow through material texture and surface manipulation. The designed model utilizes depth as an opportunity to reveal light and shadow as an aesthetic expression. The characteristics and quality of the material vary based on the orientation of the light source. The greater the depth of the aperture, the more dramatic lighting condition is experienced. Its architectural application showcases the potential of giving walls and roofs a 3-Dimensional depth to allow light to mediate between spaces. The designed experiment is an example of a designed screen or light filtration device that is able to create dynamic architectural environments through the interplay of light and shadow. 

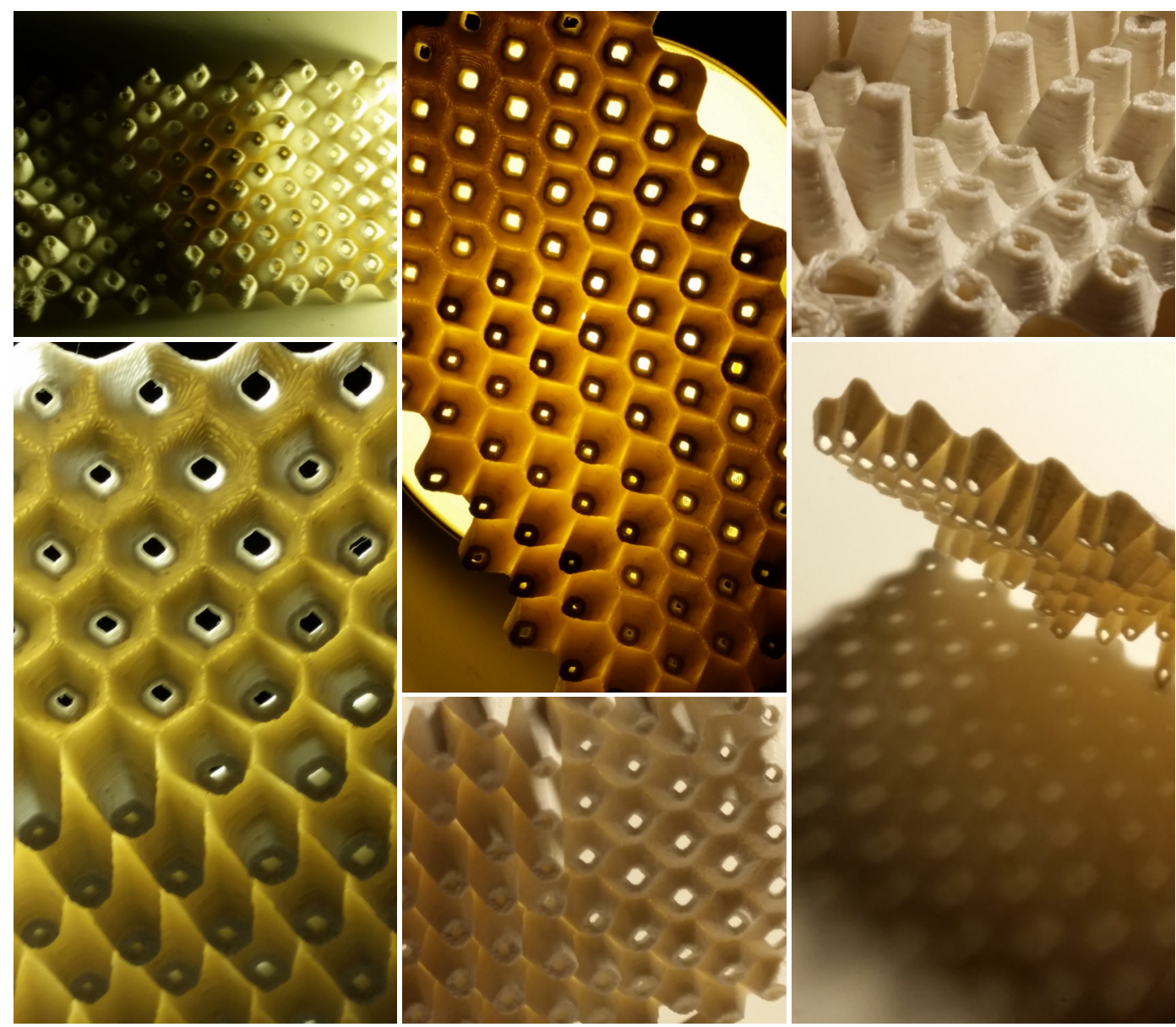

- F56

3D Printed Aperture Experiment

The experiment showcased the significance of using material and form as a method to create unique perceptual and experiential opportunity based on the quality and orientation of the light source. The designed model consists of apertures of various depth along an undulated surface, allowing for the interplay of light and shadow. The dramatic variation of light and shadow provides visual stimulus that makes the design of the model more intriguing. The translucent material (ABS Plastic) allows the object to perceptually transform from a solid form into an illuminated object, showcases the transformation of lighting effects from day to night. The amount of light transmitted through the object depends on the thickness of the material, where the joints and thin areas allow more light to permeate. The design and orientation of apertures in relation to solar movement allow for the opportunity to create lighting experiences and effects at specific moments in time. 


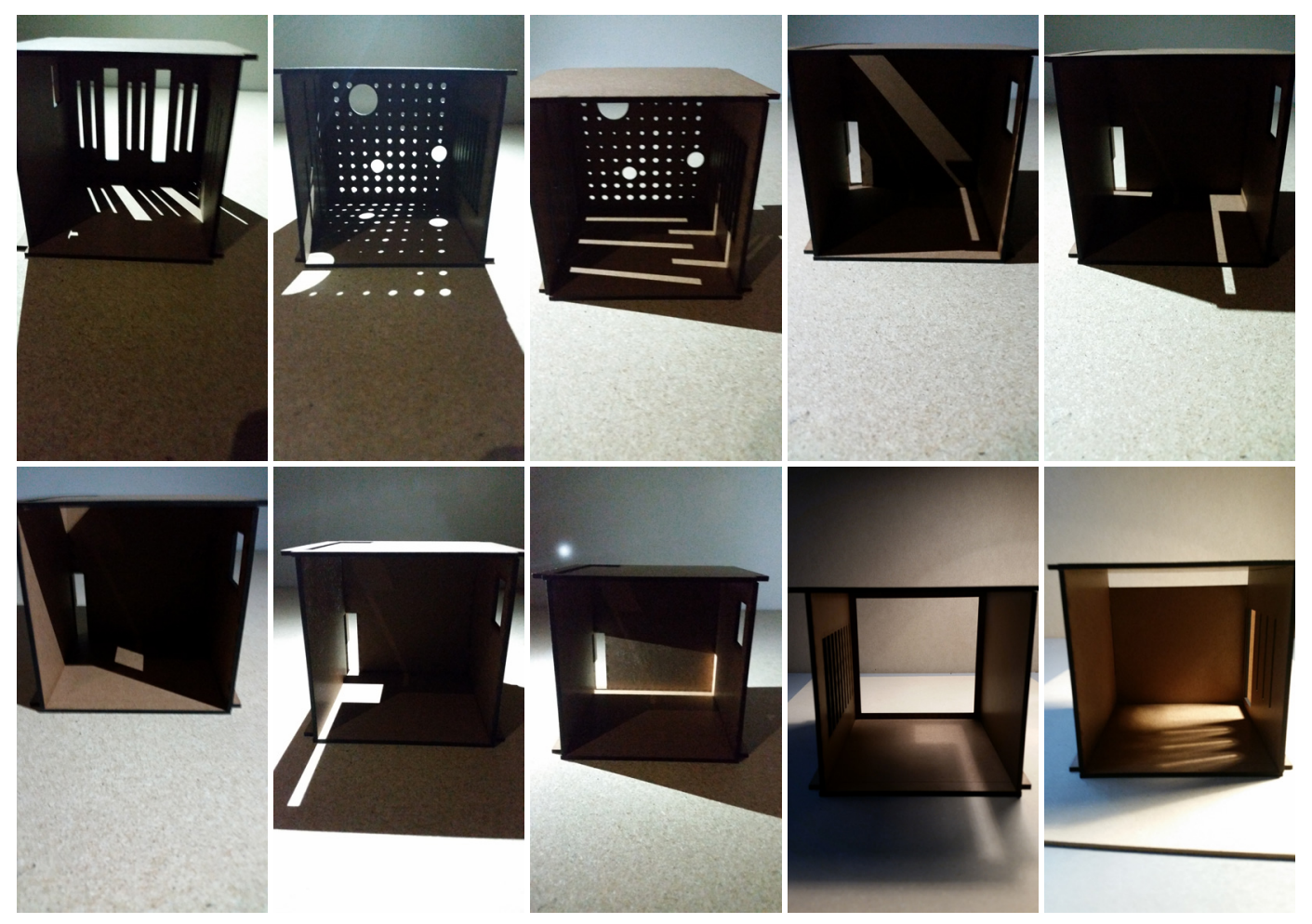

- F57

Box Light Experiment

The designed experiment utilizes aperture and light as a method to influence spatial perception and generator the architectural experience. The different openings along the walls and roof produce a distinctive lighting effects that perceptually transform the interior environment as the light changes throughout the day. The built environment has the potential to reveal light and express it as an aesthetic expression that enriches architectural environment and user experience. The experiment showcases the potential of creatively designing apertures to effect the characteristics of space. 

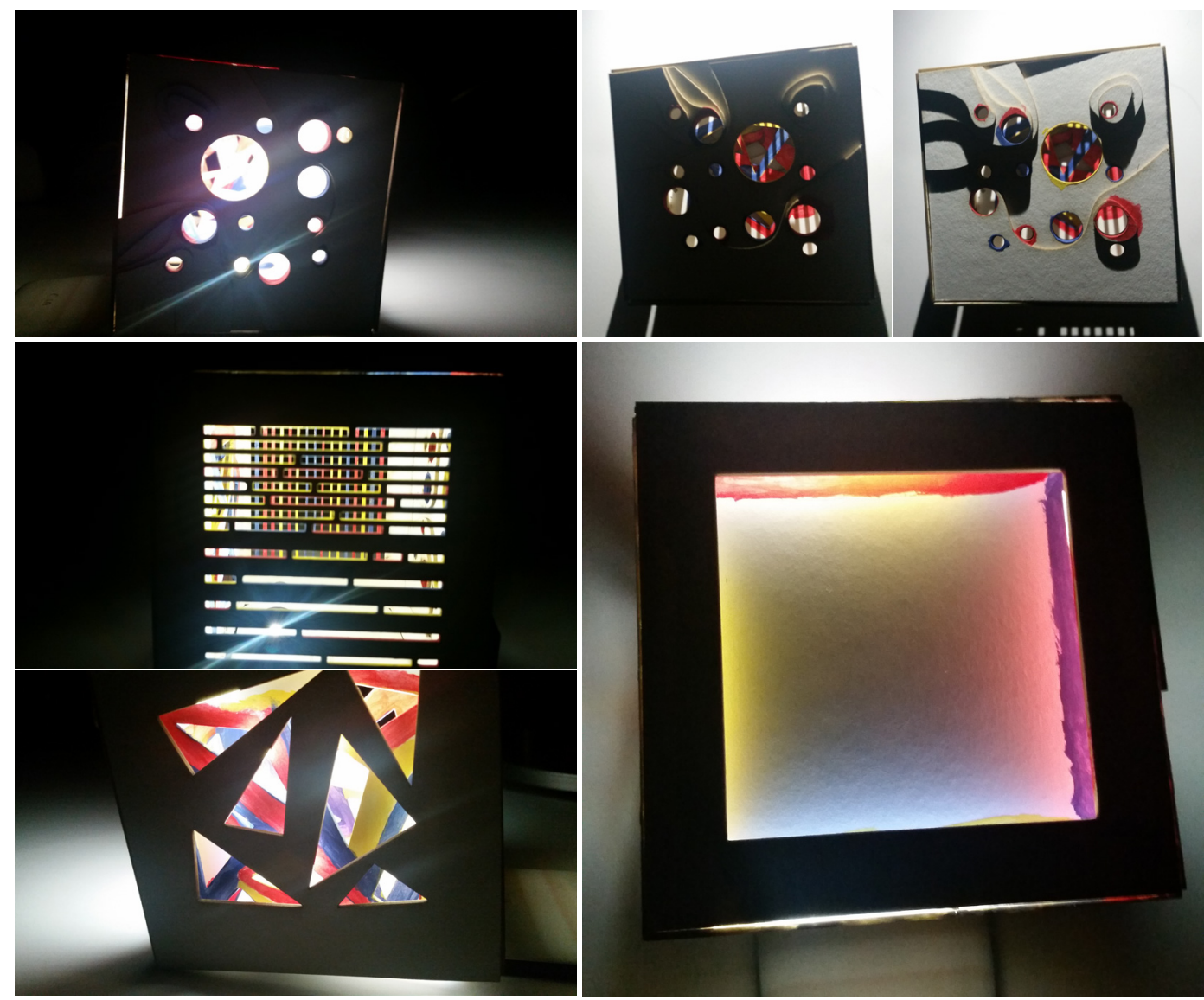

- F58

Reflected Coloured Light Experiment

This experiment attempts to express light beyond its traditional illumination of object and space. It attempts to integrate light, colour and aperture to perceptually transform the characteristics of the model. The design is a six sided box that consists of distinctively different design treatment that alter the lighting effects and experience. Colour and light are used to create perceptually stimulating surface characteristics that enriches the perception and experience of the model. The surfaces along the box are treated with 3-dimensional depth, allowing for the interplay of light and shadow through the screening and filtration of light. 

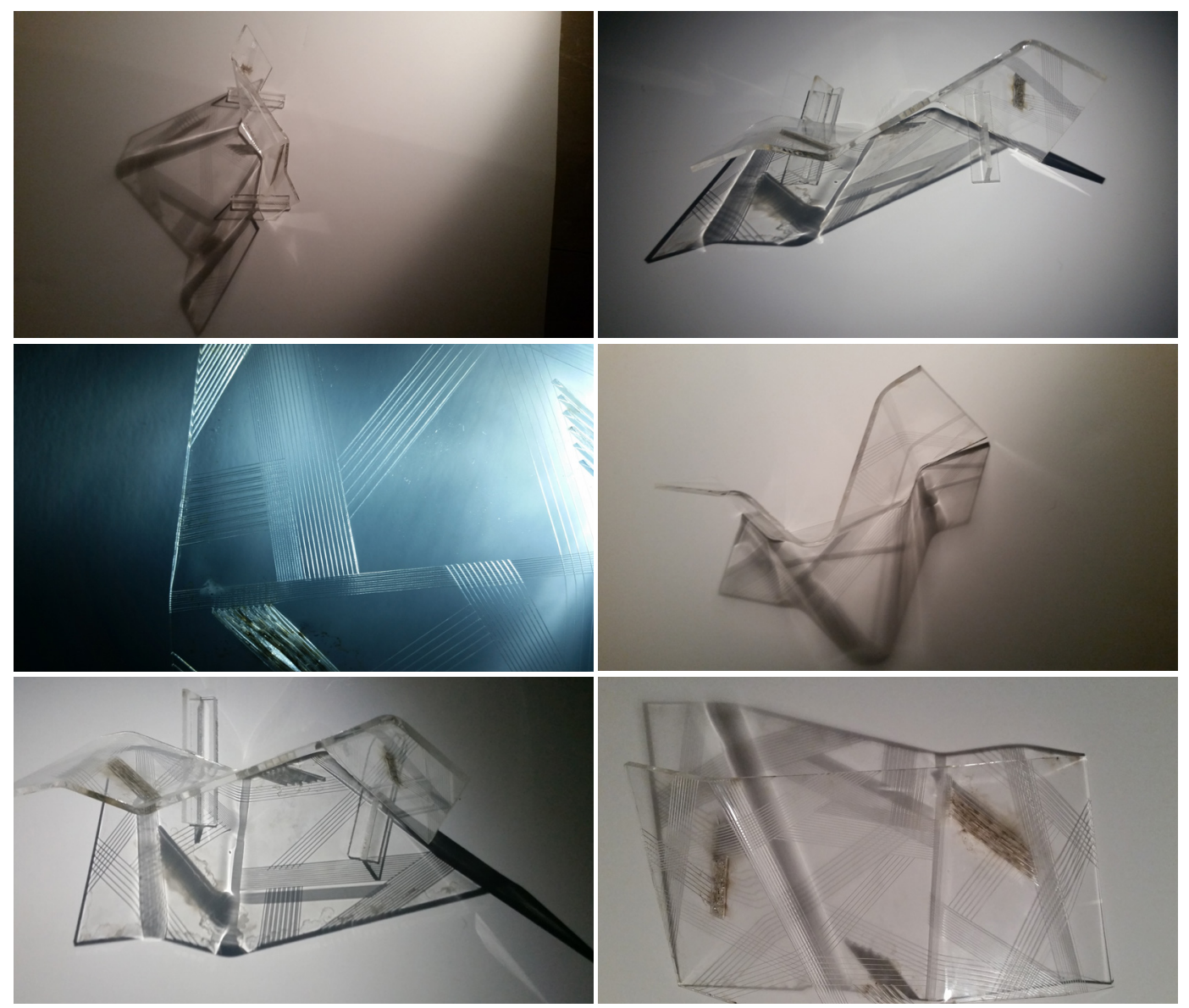

\section{- F59}

\section{Surface Manipulation}

The designed experiment showcases the significance of surface treatment in producing light and shadow effects. A flat, untouched acrylic panel is able to fully transmit light, where the etching or curving of the panel provide moments of light reflection, refraction and absorption. The produced model offers the potential to express unique lighting effects based on the location of the light source. Introducing texture along surfaces architecture allows for the interplay of light and shadow to enrich user experience and perception. 


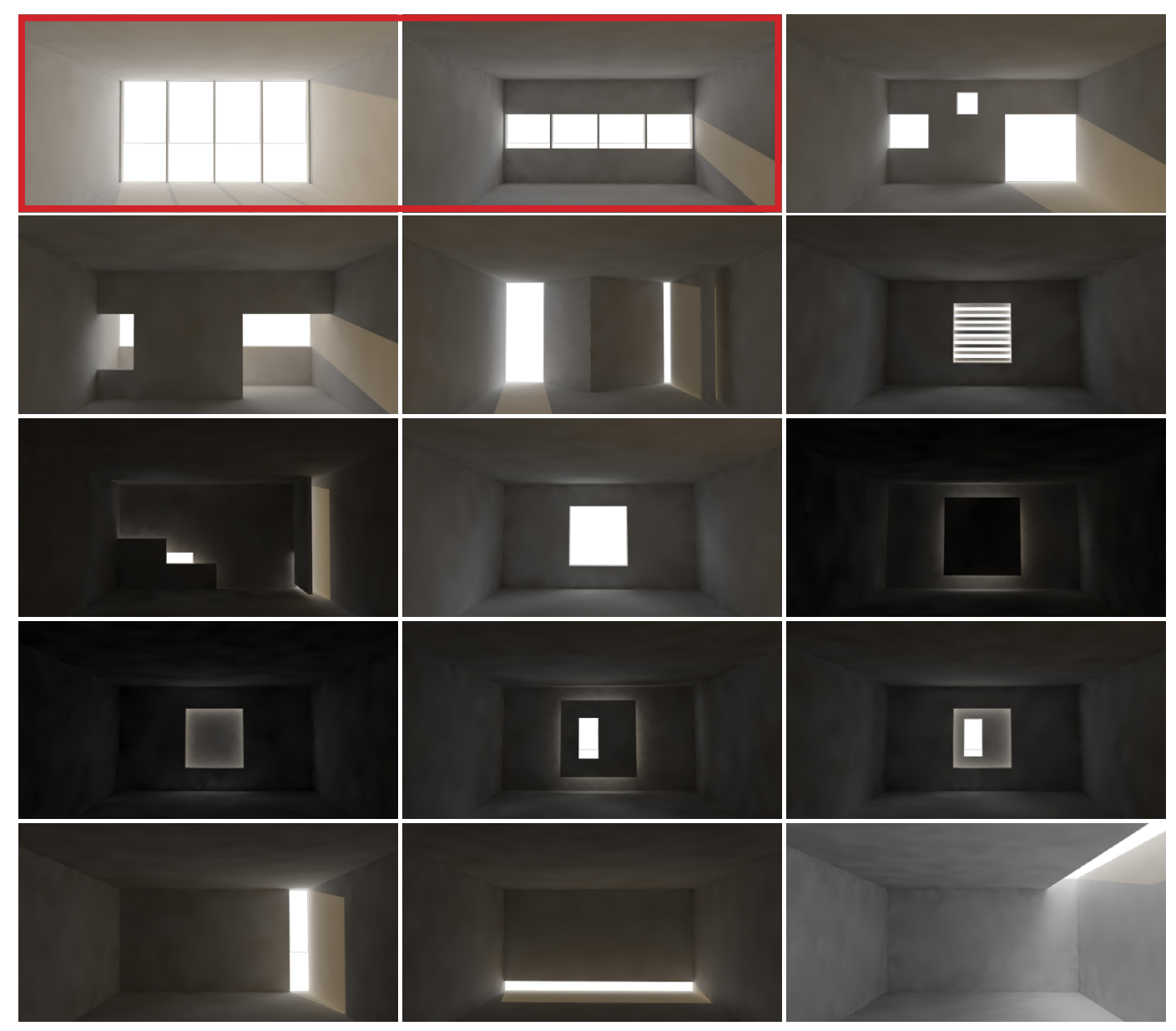

- F60

Aperture Experiment

Experiment of aperture location and lighting effects produced within space. 


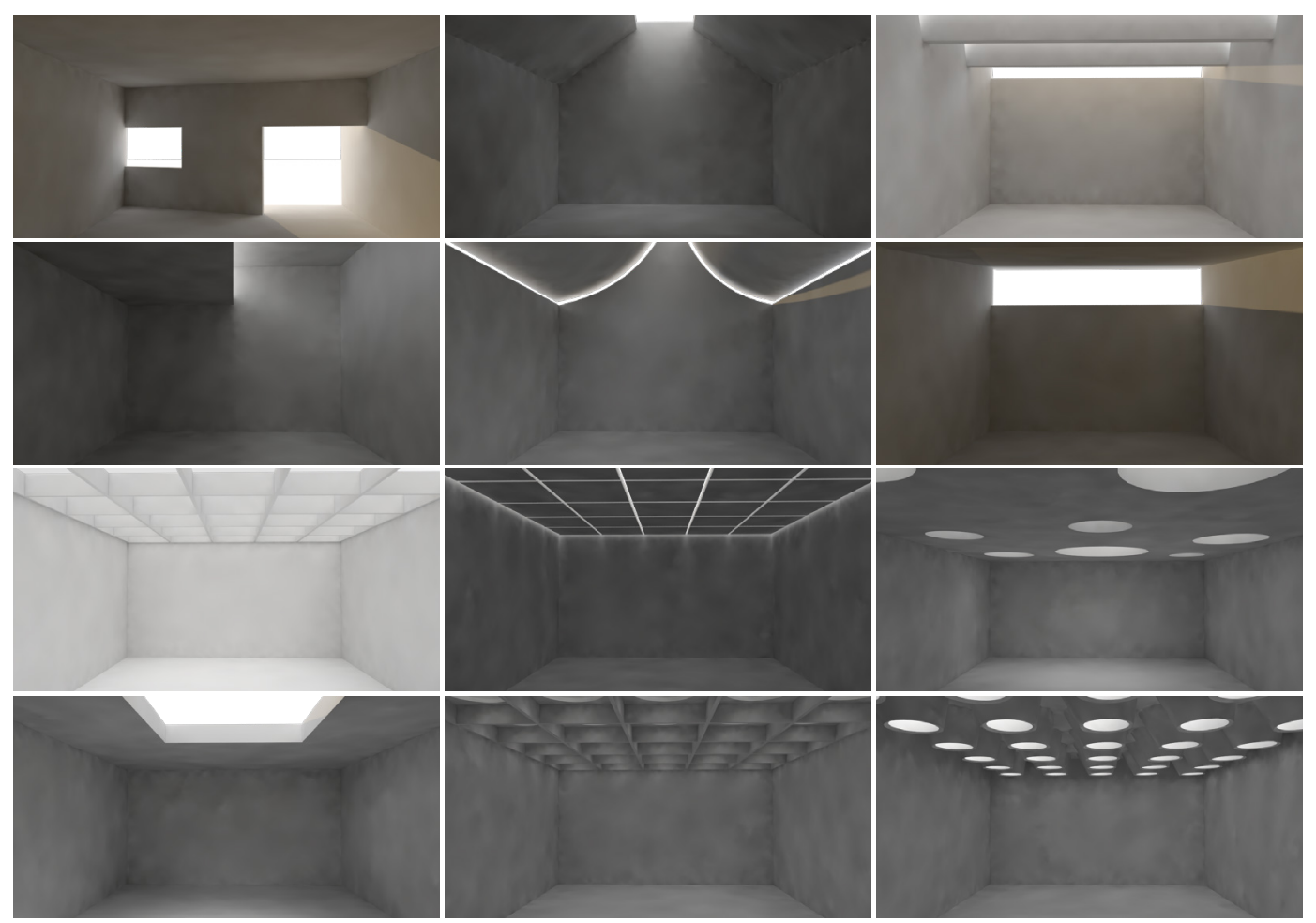

- F61

Spatial Form Experiment

Experiment of form to creatively introduce light into space and the type of spatial experience offered. 


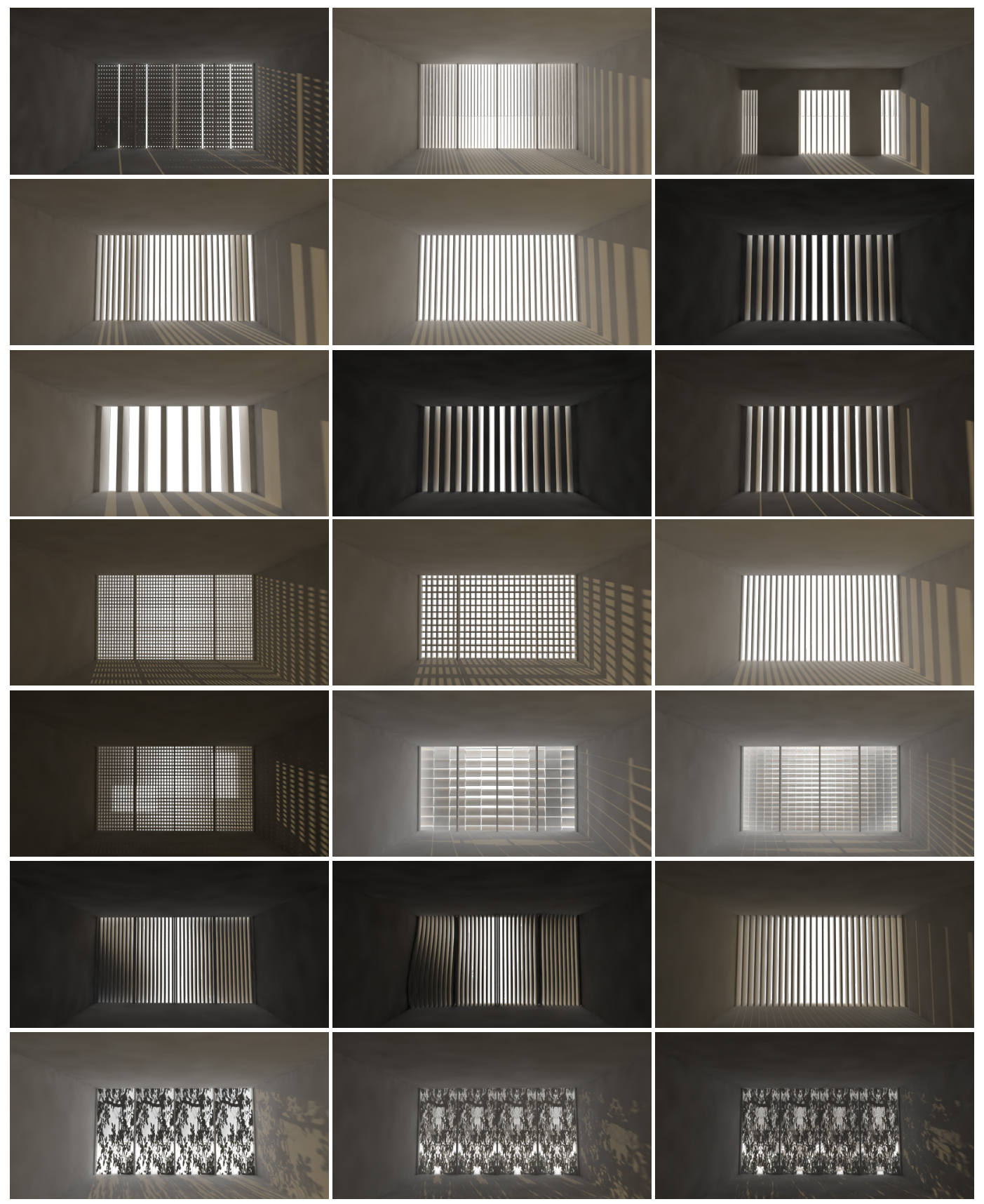

- F62

Screen and Light Filtration Experiment

Experiment of screens and light filtration devices. The examination of light and shadow variation through different screening devices and depth. 
112 | Light and Shadow Expression 


\section{Appendix B - Additional Project Drawings}

The physical models produced analyzes the transformation of sunlight and its impact on architectural environment throughout the day. The angular positioning of the sun reveals and produces different light and shadow effects that alter the perception of space. The model portrays the type of lighting condition produced. The analysis of space and lighting effects under sunlight has 


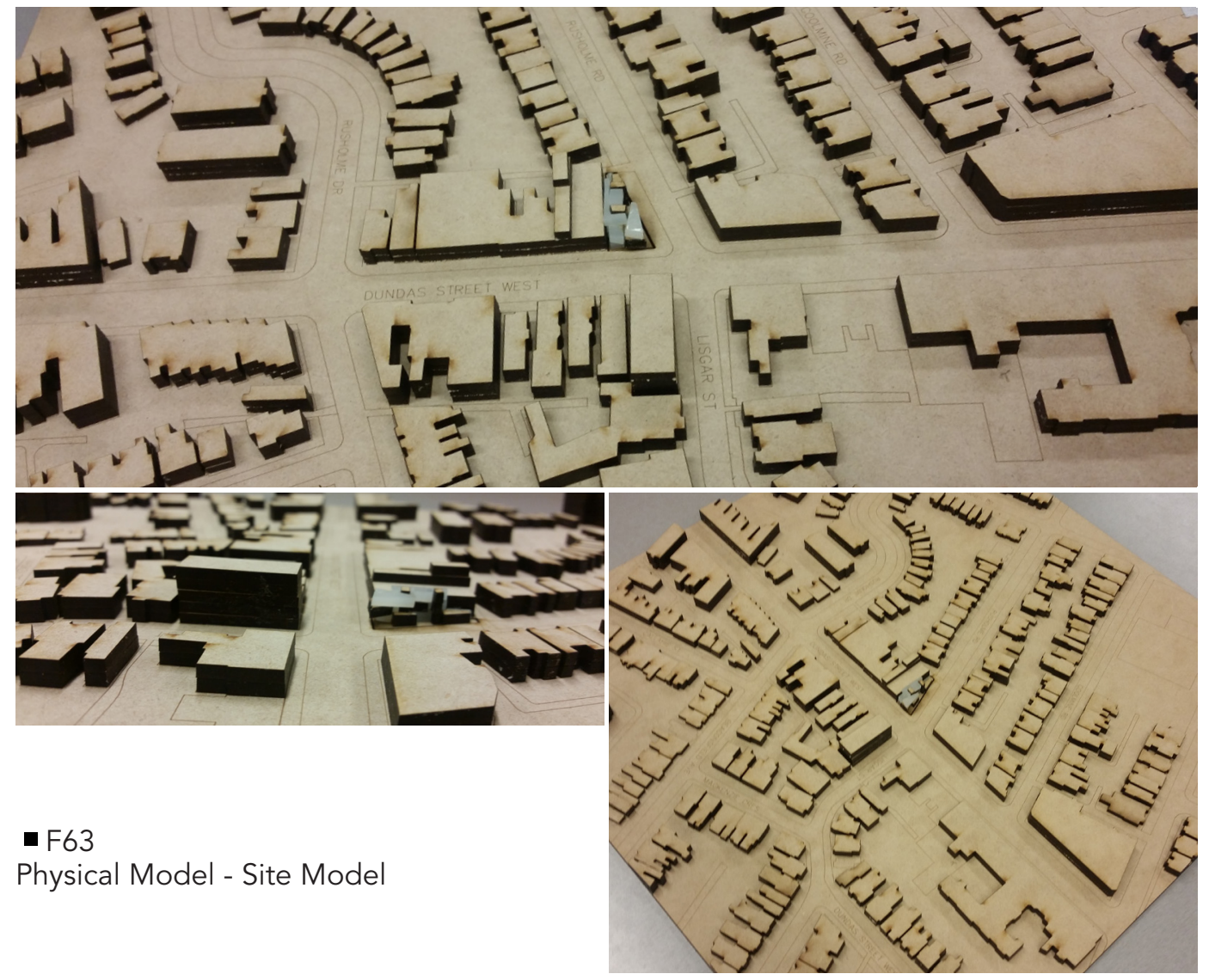

114 | Light and Shadow Expression 


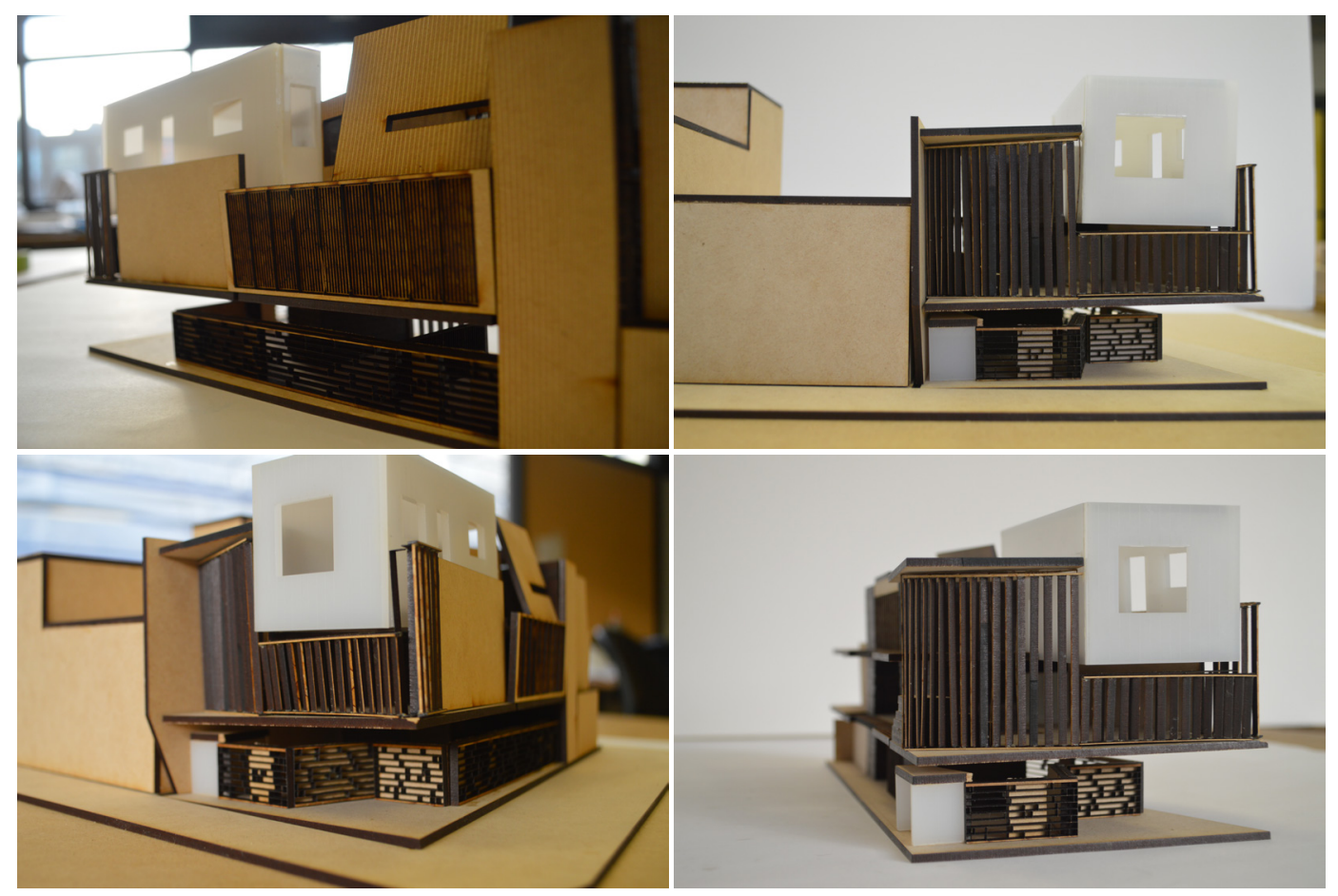

- F64

Physical Model - Massing 


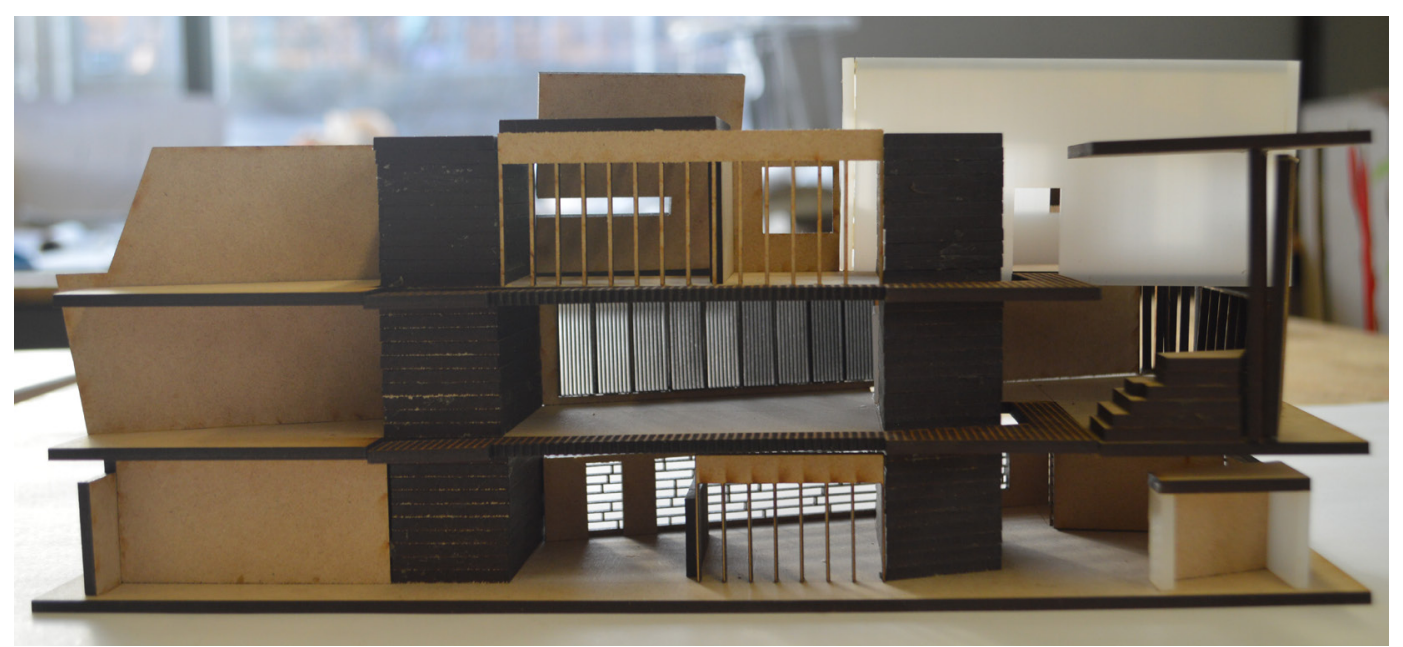

- F65

Physical Model - Section Perspective

The physical models produced examine the characteristics and quality of the designed space through the use of sunlight. The dynamic characteristics of the sun perceptually transform the characteristics of space and material. The examination of the model under different solar orientation reveals the unique design expression that enrich the architectural environment and experience. The ephemeral characteristics of the sun provide users with unique experiential moment at specific time of day. 

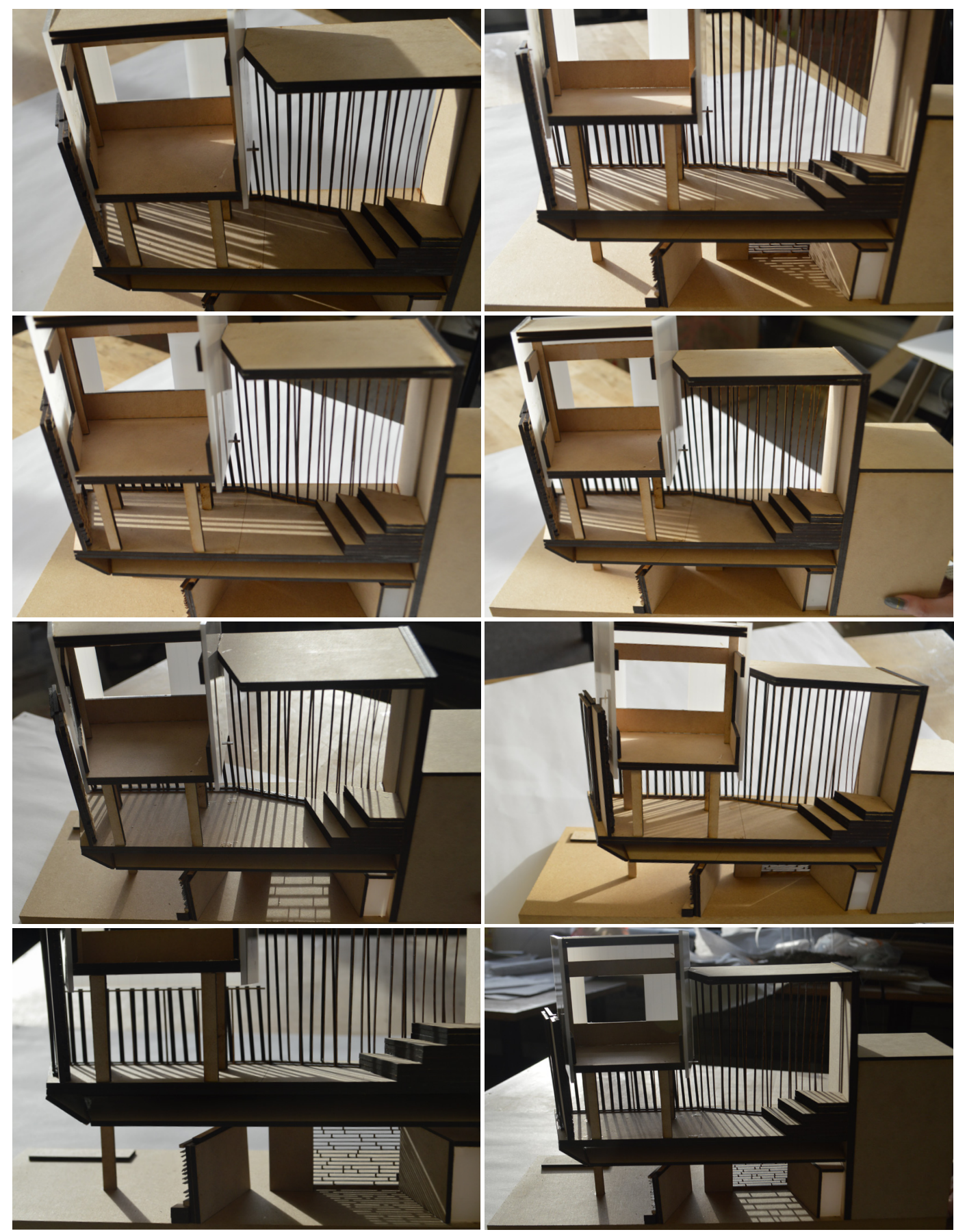

- F66

Physical Model - Detail Model 

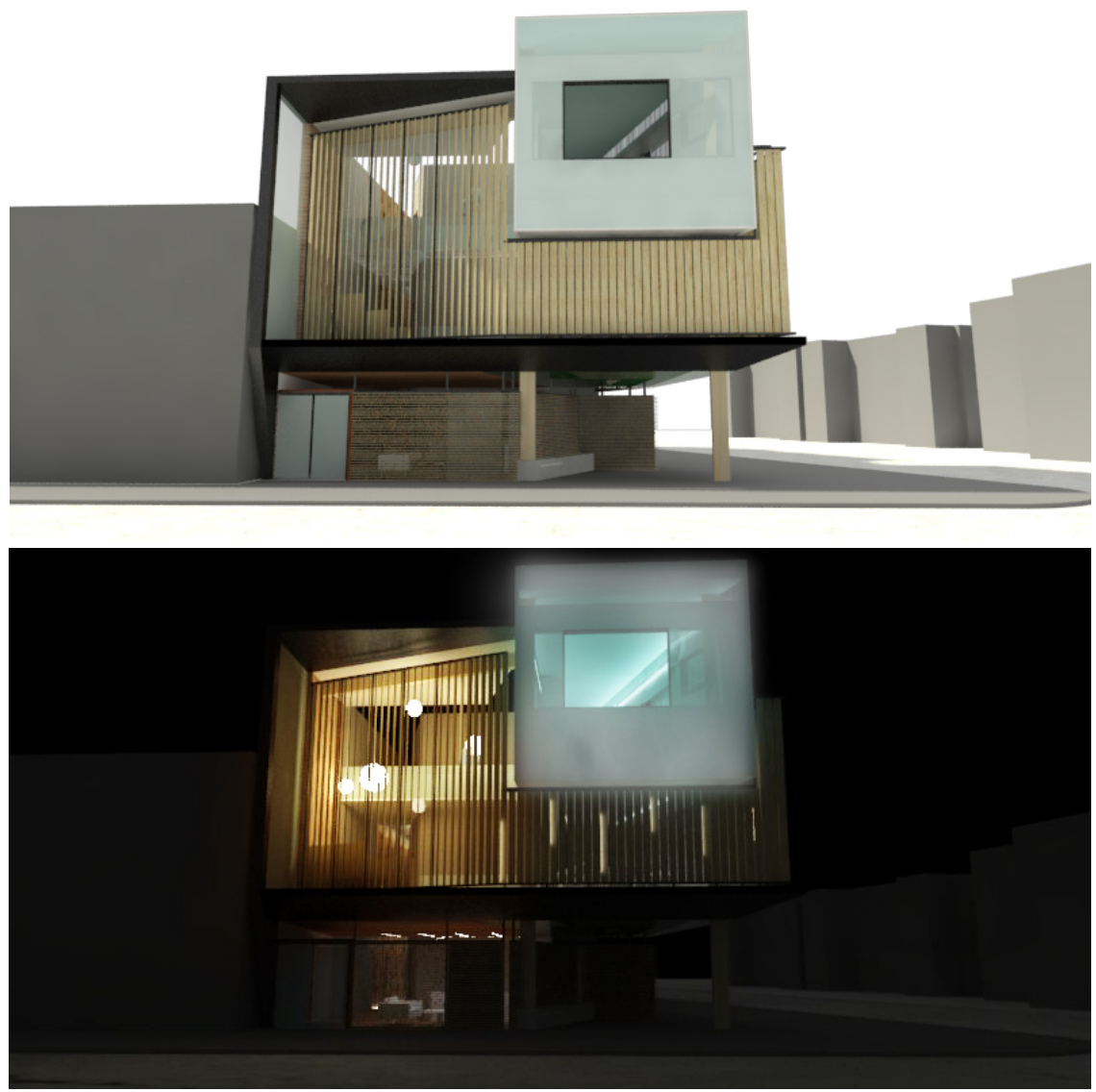

- F67

Day/Night Transformation - Dundas St. 


\section{Appendix C - Day/Night Exterior Lighting}

The transformation of light throughout the day provides new perceptual and experiential opportunity within architecture based on the type of lighting it receives. During the day, sunlight illuminates the interior of buildings, creating light and shadow effects that provide visual stimulation and enrich the experience of space. As the positioning of the sun changes, the light and shadow condition varies based on the design of the façade. Interior environments have the potential to appear dynamic and animated at specific moments of the day, creating experiences that are unique to time and location. Architecture perceptually transforms as the sun waxes and wanes throughout the day, a predictable yet variable source of illumination for architecture. The quantity, intensity, colour, orientation, etc. are all influenced by the atmosphere, producing new lighting experience and effects within the built environment.

The invention of artificial illumination provide a reliable source of illumination within architecture. Buildings appear as solid forms during the day, where at night transforms into lanterns as the light source is located within the interior environment. Artificial illumination provides architecture with an artistic display of lights along the streets within the night. The façade filters daylight into the interior space, where at night artificial illumination is filtered formed the exterior environment. Environments 

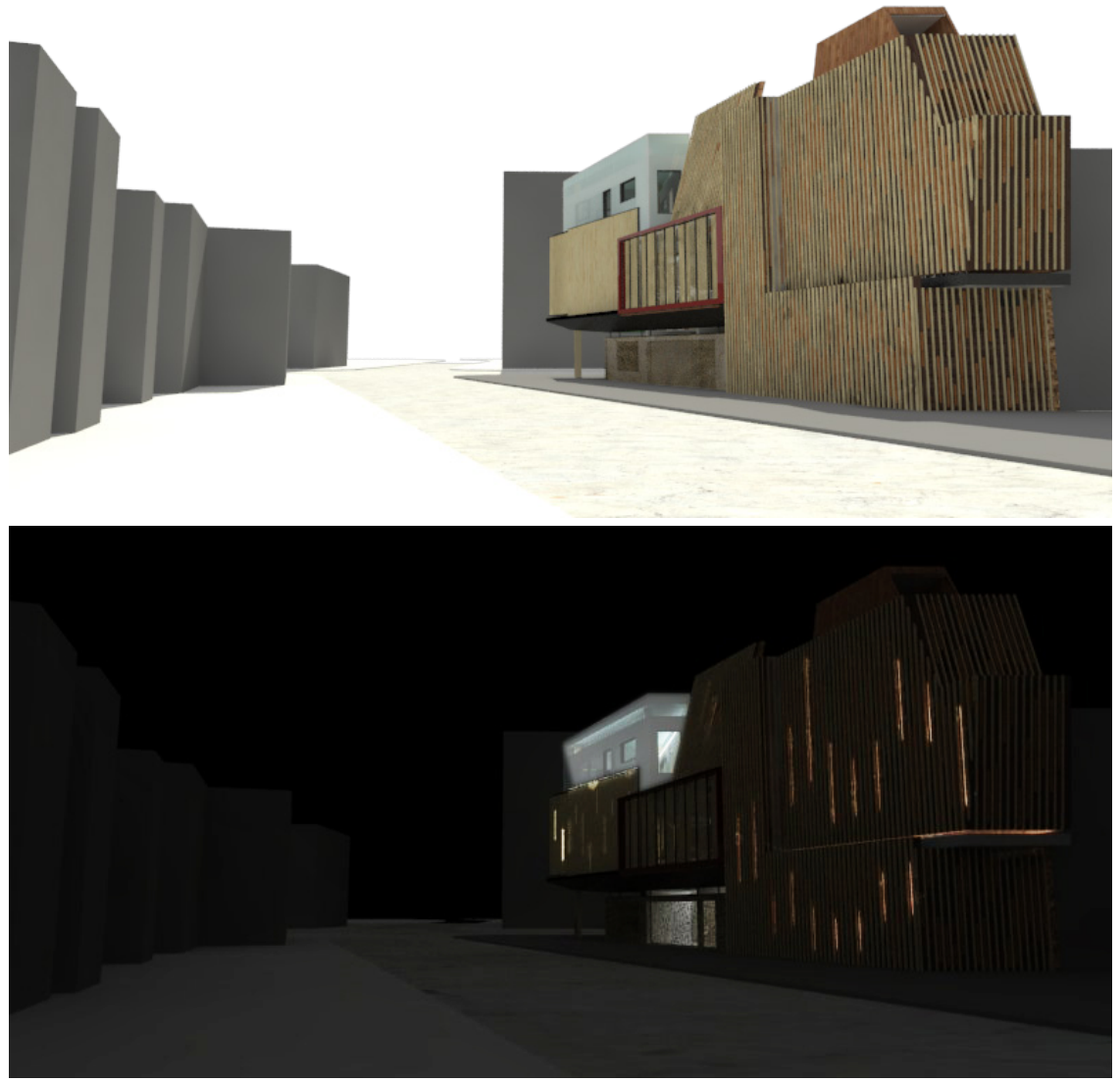

- F68

Day/Night Transformation - Rusholme Rd. 
that appear visually dominant during the day can be dissipate at night, allowing for other details and spaces to become focal point within the design. The expression of sunlight and artificial lighting has a significant role in redefining the way user perceive and experience the built environment.

Through the use of glass façades, the depth of perception is extended between the interior and exterior environments. The design uses a louvre system that are slightly angled to block or allow light into the building based on the spatial functioning. This allows for a continuous façade composition while altering the amount of light that mediates through the building. The louvre is designed to allow for more light to penetrate the collaborative space, whereas the louvres slightly rotate along the office work station to reduce direct light exposure within the space. This variation within the façade design produce different spatial lighting condition seen within the interior through the use of daylight as well as the exterior. 


\section{Bibliography}

Addington, D. M., \& Schodek, D. L. (2016). Smart materials and technologies: for the architecture and design professions. New York, NY: Routledge.

Al-Ameen, R. (2015). Softskin: Anticipating Change for the Future Architectural Envelope. Toronto: Ryerson University.

Alexander, R. (2015). Curating Light: Daylight-Centric Design for Promoting Wellness. Toronto: Ryerson University.

Ander, Gregg D. 2012. Daylighting. August 24. ttp:// www.wbdg.org/resources/daylighting.php.

Augustesen, C. (n.d.). Architecture for the Senses. Retrieved November 29, 2016, from http://iva.velux. com/competitions/international-velux-award/posts/ architecture-for-the-senses

AWARD HONOURS DAYLIGHT IN RESEARCH AND ARCHITECTURE: AND THE LAUREATES ARE... | The Daylight Site. (n.d.). Retrieved November 19, 2016, from http://thedaylightsite.com/award-honours-daylight-inresearch-and-architecture- and-the-laureates-are/

Bachelard, G., Jolas, M., \& Stilgoe, J. R. (1994). The poetics of space

Bien, H. M., \& Helle, M. (2010). International lighting 
design index 2010. Ludwigsburg: Av.

Boubekri, M. (2008). Daylighting, architecture and health building design strategies. Boston: Elsevier.

Boubekri, M. (2014). Daylighting design: planning strategies and best practice solutions. Boston: Birkhäuser.

Boyce, P. R. (1981). Human factors in lighting. Toronto: Collier Macmillan Canada.

Brandi, U. (2012). Lighting Design: Principles, Implementation, Case Studies. Basel: De Gruyter.

Brogan, J. (1997). Light in architecture. London: Academy Editions.

Chernyshov, E. (2008). Light, Dark, and all That's in Between: Revisiting the Role of Light in Architecture. Waterloo: Waterloo University

Corbusier, L. (1986). Towards a new architecture. New York: Dover Publications.

Descottes, H. (2011). Architectural Lighting: Designing with Light and Space. Princeton Architectural Press.

Eliasson, O. (n.d.). Playing with space and light. Retrieved November 29, 2016, from https://www.ted.com/talks/ olafur_eliasson_playing_with_space_and_light 
Entwistle, J. (2012). Detail in contemporary lighting design. London: Laurence King Publishing.

Frampton, K. (2002). Labour, work and architecture: collected essays on architecture and design. London I: Phaidon Press.

Heide, R. (n.d.). Why light needs darkness. Retrieved November 29, 2016, from https://www.ted.com/talks/ rogier_van_der_heide_why_light_needs_darkness

Heschong, Lisa. n.d. Daylight and Human Performance. Journal of ASHRAE 65-66.

Hobday, Richard. 2002. The Healing Sun: Sunlight and Health in the 21 st Century. Findhorn Press.

Holl, S. (2012). Color Light Time. Zurich: Lars müller.

Holl, S. (2007). House: black swan theory. New York: Princeton Architectural Press.

Holl, S. (2007). Question of Perception: Phenomenology of Architecture. William Stout Books

Holl, S. (2000). Parallax. Basel: Birkhäuser- for Architecture.

Holl, S., Carter, B., \& LeCuyer, A. W. (2005). Experiments in porosity: Steven Holl. Buffalo, NY: School of Architecture and planning, University at Buffalo.

Hopkinson, R. G., \& Schaaf, W. L. (1963). Architectural physics: lighting. London: Her Majesty's Stationery Office.

Kalff, L. (1971). Creative light. London: Macmillan.

Karcher, A. (2009). Light perspectives: between culture and technology: light, space, perspectives. Lüdenscheid: ERCO.

Kepes, G. (1985). The new landscape in art and science. Chicago: Paul Theobald and Co.

Kelly, R. (1952). Lighting as an Integral Part of Architecture.

College Art Journal,12(1), 24-30. 
Kelly, R., \& Neumann, D. (2010). The structure of light: Richard Kelly and the illumination of modern architecture. New Haven: Yale University Press in association with the Yale School of Architecture.

Koren, L. (2015). Wabi-sabi: for Artists, Designers, Poets \& Philosophers. Point Reyes, CA: Imperfect Publishing.

Krier, R. (1983). Elements of architecture. London: Architectural Design, AD Publications.

Lam, W. M. (1986). Sunlighting as formgiver for architecture. New York: Van Nostrand Reinhold.

Lam, W. M. (1992). Perception and lighting as formgivers for architecture. New York: Von Nostrand Reinhold.

Liljefors, A. (1997). Lighting and color terminology. Paper Presented at a CIE Discussion. Stockholm: Comission Internationale de l'Eclairage.

Lobell, J., \& Kahn, L. I. (1979). Between silence and light: Spirit in the architecture of Louis I. Kahn. Boulder: Shambhala.

Major, M., Speirs, J., \& Tischhauser, A. (2005). Made of light: the art of light and architecture. Basel: Birkhauser.

McCarter R. (1991). Frank Lloyd Wright: A Primer on Architectural Principles, Princeton Architectural Press, New York,

McCarter, R. (2015). Steven holl. London: Phaidon Press Limited.

Meek, C., \& Wymelenberg, K. V. (2015). Daylighting and integrated lighting design. London: Routledge, Taylor \& Francis Group.

Millet, M. (n.d.). Light and Materials. Retrieved November 19, 2016, from http://iva.velux.com/competitions/ international-velux- award/posts/light-and-materials

Millet, M. S. (1997). LIGHT REVEALING ARCHITECTURE. 
New York: Wiley.

Moholy-Nagy, L. (1947). Vision in motion. Chicago: Theobald.

Mosco, V. P., \& Holl, S. (2009). Steven Holl. Milano: Motta Architettura.

Mott, M. S., Robinson, D. H., Walden, A., Burnette, J., \& Rutherford, A. S. (2012). Illuminating the Effects of Dynamic Lighting on Student Learning. SAGE Open,2(2). doi:10.1177/2158244012445585

Pallasmaa, J., Blaufuks, D., Burnett, D., \& Holzer, P. (2016, Sept. \& oct.). Daylight Matter(s). Daylight \& Architecture, (26).

Pallasmaa, J. (2011). The embodied image: Imagination and imagery in architecture. Chichester: John Wiley \& Sons.

Pallasmaa, J. (2005). The eyes of the skin: Architecture and the senses. Chichester: Wiley-Academy

Pallasmaa, J. (2010). The thinking hand: Existential and embodied wisdom in architecture. Chichester, U.K.: Wiley.

Pallasmaa, J. (n.d.). Dwelling in light - tactile, emotive and life-enhancing light. Retrieved November 29, 2016, from http://www.velux.com/article/2016/dwelling-in-light

Pallasmaa, J. "Hapticity and Time: Notes on a Fragile Architecture." The Architectural Review, 2000: 78ख84.

Plummer, H. (2012). Nordic light: Modern Scandinavian architecture. New York: Thames \& Hudson.

Plummer, H. (2009). The Architecture of Natural Light. New York: The Monacelli Press, a division of Random Hous, Inc.

Ramos, E. V. (2015). Light in architecture: the intangible material. London: RIBA Publishing. 
Richards, B. (2006). New glass architecture. London: Laurence King.

Riley, T. (2003). Light construction. New York: The Museum of Modern Art.

Rockcastle, S., \& Andersen, M. (2013). Architectural Context. Annual Dynamics of Daylight Variability and Contrast SpringerBriefs in Computer Science, 23-35. doi:10.1007/978-1-4471-5233-0_3

Safont-Tria, J., Kwinter, S., \& Holl, S. (2012). Steven Holl: Color, light, time. Zurich, Switzerland: Lars Müller.

Schoof, J. (n.d.). Designed for all senses. Retrieved May 12, 2017, from http://www.velux.com/daylightand-architecture/evolving-architecture/designed-forall-senses

Schröpfer, T., \& Carpenter, J. (2011). Material design: informing architecture by materiality. Basel: Birkhäuser.

Shank, J. (2013). Weak Material: Light, Time, Location and the Stuff of Architecture. Toronto: Ryerson University.

Steane, M. A., \& Steemers, K. (2004). Environmental diversity in architecture. New York: Spoon Press.

Steane, M. A. (2011). The architecture of light: recent approaches to designing with natural light. London: Routledge.

Tanizaki, J. (1977). In praise of shadows. New Haven, CT: Leete's Island Books.

Tsachrelia, D. (2014, March 14). : Architecture needs a Beginning. Retrieved November 29, 2016, from http://www.c-o-l-o-n.com/2_1holl.html

Urban Density Done Right Ideas on densification of cities and other communities. Karlskrona: Boverket, 2017. Print. 
Verges, M. (2008). Light in architecture. Place of publication not identified: Natl Book Network.

Venturi, R., \& Scully, V. J. (1966). Complexity and contradiction in architecture. New York: Museum of Modern Art

Vesely, D. (2004). Architecture in the age of divided representation: The question of creativity in the shadow of production. Cambridge, MA: MIT Press.

Whiting, S. (2012). Beyond surface appeal: literalism, sensibilities, and constituencies in the work of James Carpenter. Cambridge, MA: Harvard University Graduate School of Design.

Winchip, S. M. (2017). Fundamentals of lighting. New York: Fairchild Books.

Zumthor, P. (1998). Thinking Architecture. Baden: Lars Müller.

Zumthor, P. (2006). Atmospheres: Architectural Environments, surrounding objects. Basel: Birkhäuser. 